\title{
Osteología craneal comparada de tres especies de lenguado del género Paralichthys (Pleuronectiformes, Paralichthyidae) del Atlántico suroccidental
}

\author{
Comparative cranial osteology of three species of the flatfish genus Paralichthys \\ (Pleuronectiformes, Paralichthyidae) from the southwestern Atlantic
}

\author{
JUAN M. DÍAZ DE ASTARLOA
}

\begin{abstract}
Consejo Nacional de Investigaciones Científicas y Técnicas, Departamento de Ciencias Marinas, Facultad de Ciencias Exactas y Naturales, Universidad Nacional de Mar del Plata, Funes 3350 B7602AYL, Mar del Plata, Argentina; e-mail: astarloa@mdp.edu.ar
\end{abstract}

\begin{abstract}
RESUMEN
Se realiza un análisis osteológico comparativo del esqueleto craneal en Paralichthys isosceles, $P$. orbignyanus y $P$. patagonicus, tres especies de lenguados presentes en el Atlántico suroccidental. Se describen las características de los componentes óseos y se detallan las diferencias morfológicas y morfométricas encontradas, con el fin de aportar elementos diagnósticos para la identificación taxonómica de las tres especies de Paralichthys. Se utilizaron técnicas de desarticulación de esqueletos, tanto en ejemplares frescos congelados, como en especímenes conservados en líquidos fijadores. También fueron usadas técnicas de clareado y tinción diferencial para ejemplares de pequeño tamaño. Se observaron potenciales características diagnósticas para la discriminación de las especies en los huesos paretmoides derecho, lacrimal izquierdo, anguloarticular, mesopterigoides, los otolitos y el número de dientes del dentario. Se discuten las relaciones entre los huesos y se comparan sus características con las halladas en otras especies del género Paralichthys, en particular, y en otros Pleuronectiformes en general.
\end{abstract}

Palabras clave: Pleuronectiformes, Paralichthys, osteología, esqueleto cranial, Atlántico suroccidental.

\begin{abstract}
An osteological analysis of the cranial skeleton of the southwestern Atlantic flatfish species Paralichthys isosceles, $P$. orbignyanus and $P$. patagonicus was carried out based on comparative morphology and morphometrics of the bones. The objective was to provide osteological evidence of diagnostic value for a correct identification of the flatfish species. Methods for preparing disarticulated skeletons were used for both dissected fresh-frosen and alcohol-preserved specimens. Also methods of clearing and staining for bone and cartilage were applied for small fish specimens. Potentially diagnostic features were found to distinguish among species in the right parethmoid, left lachrymal, angulo-articular, mesopterygoid, the otoliths, and the number of dentary teeth. Relationships between bones are discussed and bone characteristics are compared with those found in other Paralichthys species and in other Pleuronectiform species.
\end{abstract}

Key words: Pleuronectiformes, Paralichthys, ostelogy, cranial skeleton, southwestern Atlantic.

\section{INTRODUCCIÓN}

En el Atlántico suroccidental la gran similitud exterior de los lenguados es un obstáculo serio para reconocerlos. De ahí que su biología sea poco conocida y sobre su aprovechamiento no se ha llegado aún a dar normas o recomendaciones. Diversos autores proponen diferentes características para distinguir las especies del género Paralichthys. Jordan \& Goss (1889) y
Jordan \& Evermann (1898) brindan proporciones corporales, relaciones morfológicas entre diversas partes del cuerpo, conteo de elementos merísticos y patrones de coloración, como elementos diagnósticos. Norman (1934) aporta como caracteres de importancia taxonómica la morfología y el tamaño de las escamas en ambos lados del cuerpo, presencia de escamas suplementarias, estructura y dirección de las ramas accesorias 
del sistema de la línea lateral, presencia o ausencia, tamaño y número de ciegos pilóricos. El mismo autor aclara que los caracteres más comúnmente y ampliamente utilizados son los merísticos (número de radios de las aletas dorsal, anal, pectoral, pélvica y caudal, número de escamas en hileras longitudinales y transversales, número de poros de la línea lateral y número de vértebras). El número de rastrillos branquiales (branquispinas), en combinación con diferencias en el patrón de coloración también son usados como herramientas taxonómicas (Ginsburg 1952). Pequeño \& Plaza (1987), con referencia a los lenguados chilenos del género Paralichthys afirman: "presentan una coloración y una morfología externa bastante parecida, de modo que aspectos de proporcionalidad en su morfometría no siempre son prácticos y fáciles de usar para la diferenciación de las especies. Más aún, es muy posible que haya cambios de patrones de coloración durante el ciclo de vida y ellos son tan desconocidos como los cambios proporcionales de las diferentes estructuras externas. Sin embargo, los elementos merísticos pueden ser de gran utilidad". Los caracteres merísticos han sido los elementos más utilizados en los estudios taxonómicos sobre los lenguados de Chile (Pequeño \& D' Ottone 1987).

Los caracteres osteológicos también han sido utilizados en los peces como fundamento para establecer criterios taxonómicos para distinguir especies (Collette \& Chao 1975, Collette \& Gillis 1992), pero en el caso de los Pleuronectiformes su uso ha sido más limitado. Woolcott et al. (1968) realizan un estudio descriptivo y comparativo de tres especies de Paralichthys presentes en el Atlántico noroccidental $[P$. dentatus (Linnaeus, 1766), $P$. lethostigma Jordan \& Gilbert, 1884 y $P$. albigutta Jordan \& Gilbert, 1882]. Balart (1985) discute el complejo hipural de Paralichthys olivaceus (Temminck \& Schlegel, 1846) con los Paralichthys spp. descriptos por Woolcott et al. (1968). Díaz de Astarloa (1991) efectúa una comparación del sincráneo y complejo caudal en dos formas nominales de Paralichthys: $P$. patagonicus Jordan, en Jordan \& Goss, 1889 y P. bicyclophorus Miranda Ribeiro, 1915.

Por otro lado, la literatura menciona la utilidad de las características osteológicas en la identificación de los ítemes presa en contenidos estomacales de animales ictiófagos (Watt et al. 1997, Favero et al. 2001). Por ejemplo, los otolitos, entre otros huesos, constituyen excelentes elementos diagnósticos para la identificación de peces (Volpedo \& Echeverría 2000, 2001, 2003).

La identificación de las especies del género Paralichthys en el Atlántico suroccidental ha sido basada principalmente en diferencias morfológicas externas (proporciones corporales y conteos de elementos merísticos), sin embargo estas características muchas veces no son definitorias debido a diferentes grados de superposición encontrados entre las especies (Díaz de Astarloa 1994), o son de poca utilidad cuando los animales se hallan en un estado avanzado de descomposición, o cuando, una vez ingeridos, son rápidamente degradados por las secreciones digestivas de los estómagos de sus predatores. De esta manera las características esqueletarias constituyen elementos diagnósticos de importancia a la hora de la identificación taxonómica de las especies. Por otro lado, el sistema óseo constituye el fundamento sobre el cual se apoya hoy en día la definición de los grandes grupos y la discusión de afinidades y diferencias entre los mismos (Cervigón 1980). La descripción de nuevas especies para la ciencia, en la mayoría de los casos, se fundamenta en variaciones de características morfométricas y/o merísticas encontradas entre individuos que se supone pertenecen a distintas entidades específicas, sin embargo no se analiza si esas diferencias se deben más a variaciones intraespecíficas o si efectivamente tienen valor específico (Díaz de Astarloa 1995). Miranda Ribeiro (1915) describe a Paralichthys bicyclophorus para aguas brasileñas y argentinas sobre la base de características morfológicas externas y patrón de coloración. Esta especie ha sido considerada como válida hasta recientemente (Ringuelet \& Arámburu 1960, Roux 1973, Lema et al. 1980, Menni et al. 1984), sin embargo, el análisis osteológico comparativo (Díaz de Astarloa 1991) y la examinación de los ejemplares tipo (Díaz de Astarloa 1996) no dieron evidencias de que constituyera una nueva especie.

En este trabajo se pretende fundamentar la hipótesis de que las tres especies nominales del género Paralichthys $[P$. isosceles Jordan,1891, $P$. orbignyanus (Valenciennes, 1839) y $P$. patagonicus] simpátricas del Atlántico suroccidental, se distinguen entre sí por los elementos constituyentes del esqueleto craneal, 
y que la osteología es una gran fuente de evidencia para confirmar la validez de estas especies nominales.

La descripción comparativa de los componentes del esqueleto craneal en las tres especies del género Paralichthys tiene como objetivo aportar elementos diagnósticos que contribuyan a una mejor identificación taxonómica de las especies y a la utilidad en la identificación de los elementos óseos en contenidos estomacales de animales ictiófagos.

\section{MATERIALES Y MÉTODOS}

\section{Material examinado}

Se utilizaron 35 ejemplares de Paralichthys patagonicus de tallas comprendidas entre $25 \mathrm{y}$ $48 \mathrm{~cm}, 10$ ejemplares de $P$. orbignyanus, entre 39 y $103 \mathrm{~cm}$, ambas especies colectadas en la Zona Común de Pesca Argentino-Uruguaya (343' $\mathrm{S}$ y $39^{\circ} 30^{\prime} \mathrm{S}$ y entre los 40 y $100 \mathrm{~m}$ de profundidad), 13 individuos adicionales de $P$. orbignyanus procedentes de la laguna costera Mar Chiquita, situada a los $37^{\circ} 46^{\prime} \mathrm{S}$ y $57^{\circ} 27^{\prime}$ $\mathrm{O}$, al NE de la ciudad de Mar del Plata, en la Provincia de Buenos Aires. También fueron analizados 23 ejemplares de $P$. isosceles con un rango de tallas de 13 a $37 \mathrm{~cm}$ y capturados en aguas patagónicas entre los $44^{\circ} 21^{\prime} \mathrm{S}$ y $46^{\circ} 54^{\prime}$ $\mathrm{S}$, a una profundidad entre 50 y $80 \mathrm{~m}$.

Como material suplementario, y con el objetivo de realizar un análisis comparativo, se examinó material depositado en colecciones ictiológicas, y que se indica a continuación. Se siguió a Leviton et al. (1985) para el uso de los acrónimos de las instituciones.

Paralichthys adspersus (Steindachner, 1867): USNM 128153, 2 (154, $167 \mathrm{~mm} \mathrm{LS})$, La Lagunilla, Perú; 25 jun 1941. MNHN 20010510, 1 (143 mm LS), Boca del Río, Perú, 26 nov 1997. MNHN 1907-0343, 1 (320 mm LS), Antofagasta, Chile. MNHN 1907-0345, 1 (231 mm LS), Antofagasta, Chile. MNHN 1907-0344, 1 (218 mm LS), Antofagasta, Chile. MNHN 1907-0346, 1 (210 mm LS), Antofagasta, Chile.

Paralichthys aestuarius Gilbert \& Scofield, 1898: USNM 48128, 2 sintipos (158, $176 \mathrm{~mm}$ LS), Shoal Point, Golfo de California, boca del Río Colorado, Sonora, México.

Paralichthys albigutta: USNM 4887, sintipo (123 mm LS), Laguna Grande, Pensacola,
Florida, USA. USNM 30818, sintipo (162 mm LS), Laguna Grande, Pensacola, Florida, U.S.A. USNM 21279: neotipo (234 mm LS).

Paralichthys brasiliensis (Ranzani, 1842): MZUB 1408, holotipo. MNHN 1999-0437, 1 (273 mm LS), Bahia, Brasil. MNRJ 1932, 1 (232 mm LS), Mercado de Bahia, Brasil. MZUSP 72303, 2 (112, 122 mm LS), Rio Curuça, Ilha de São Luis, Brasil, 28 julio 1982. MZUSP 72304, 1 (102 mm LS), Porto Angra dos Reis, Río de Janeiro, Brasil, enero 1982. MCZ 4669, 1 (173 mm LS), Bahia, Brasil. Porto Seguro, 1886. MCZ 11407, 2 (80, 85 mm LT), Brasil, Espiritu Santo. Rio São Matheus. Noviembre 1865. MCZ 11409, 1 (248 mm LS), Brasil. Rio de Janeiro. Rio Itabapoana, 1865. MCZ 11404, 1 (218 mm LS), Brasil, Bahia. Camamu. Thayer Expedition. 135ㄱ' S, 03911' O, mayo 1866. MCZ 11403, 1 (174 mm LS), Brasil. Para Ponta Curuça. 0043' S 04752' O Thayer expedition. 29 febrero 1866. MCZ 11408, 1 (342 mm LS), Brasil. Espiritu Santo, Rio São Mateus, 1844' S, 0395' O, Thayer Expedition, noviembre 1865. FAKU 37967, 1 (210 mm LS), Río de Janeiro, 1975.

Paralichthys californicus (Ayres, 1851): USNM 64039, 2 (130, 151 mm LS), San Diego, California, 15 marzo 1894. MNHN 1896-0151, 1 (226 mm SL), Colombia, Magdalena. MNHN A 3297, 2 (115, $139 \mathrm{~mm}$ LS), USA, California, Santa Barbara, 1881.

Paralichthys dentatus (Linnaeus, 1766): USNM 127184, 2 (151, 182 mm LS), bahía de Chesapeake. USNM 187286, 1 (134 mm LS), Delaware. MNHN 1999-0459, 1 (132 mm LS), Antillas, Cuba. MNHN 1999-0462, 1 (191 mm LS), USA, Virginia, Potomac. MNHN A 5186, 1 (150 mm LS), USA, South Carolina, Charleston. MNHN A 9964, 3 (243-287 mm LS), USA, New York, 1820. MNHN 1999-0469, 1 (134 mm LS), USA, New York. MNHN 1999-0464, 1 (196 mm LS), USA, South Carolina. MNHN 1999-0463, 1 (228 mm LS), USA, New York. MNHN 19990461, 4 (116-284 mm LS), USA, New York. MNHN 1999-0999, 2 (174, 242 mm LS), USA, South Carolina, Charleston.

Paralichthys isosceles: MACN 2520; 1 (106 $\mathrm{mm}$ LS); $45^{\circ} 08^{\prime} \mathrm{S}-66^{\circ} 28^{\prime} \mathrm{O}$. MACN 6312; 3 (87-198 mm LS); 37³7' S - 56²14' O; Buque "Hero"; 26 agosto 1971. MACN 6468; 1 (185 mm LS); frente a Rawson, Chubut. INIDEP 145;

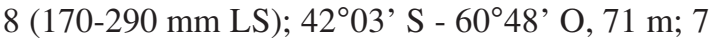
enero 1971. INIDEP 146; 1 (276 mm LS); 42 ${ }^{\circ} 03^{\prime}$ S - 60² $48^{\prime} \mathrm{O}, 71 \mathrm{~m} ; 7$ enero 1971. MZUSP 
72370; 2 (149, $171 \mathrm{~mm} \mathrm{LS}) ; 32^{\circ} 22^{\prime} \mathrm{S}, 51^{\circ} 20^{\prime} \mathrm{O}$; N. Oc. Besnard, estación 1756; 22 abril 1972. MZUSP 72377; 1 (110 mm LS); 2843' S, 48³0' O; Prof. Besnard, estación 1191; 18 agosto 1970. MZUSP 72379; 2 (117, $120 \mathrm{~mm} \mathrm{LS}) ; 33^{\circ} 29^{\prime} \mathrm{S}$, 51 ${ }^{\circ} 28^{\prime}$ O; N. Oc. Besnard, estación 1748 Bt.; 20 abril 1972. MZUSP 723321 (177 mm LS); 293' S, 48 $57^{\prime}$ O; N. Oc. Besnard, estación 1706; 6 abril 1972. USNM 43335; tipo, 1 (211 mm SL); Bahía, Brasil; 1887. USNM 43368; 1 paralectotipo (204 mm LS); Bahía, Brasil R/V Albatross; 1887. USNM 43371; lectotipo, 1 (203 mm LS); Bahía; R/V Albatross; 1887.

Paralichthys lethostigma Jordan \& Gilbert, 1884: USNM 21279, neotipo (234 mm LS), río St. John Florida; sin fecha de colecta. MNHN 1999-0458, 2 (98, 151 mm LS), USA, New York.

Paralichthys microps (Günther, 1881): FAKU CP 624, 1 (290 mm LS), erróneamente identificado como $P$. adspersus; sin fecha de colecta. FAKU CP 622, 1 (283 mm LS), erróneamente identificado como $P$. adspersus; sin fecha de colecta. FAKU 117696, 1 (208 mm LS), Patagonia chilena, 18 septiembre 1977, Akebono-maru, erróneamente identificado como P. brasiliensis. FAKU 117702, 1 (257 mm LS), Patagonia chilena, 18 septiembre 1977, Akebono-maru, erróneamente identificado como P. brasiliensis. FAKU 117417, 1 (247 mm LS), Patagonia chilena, 5 septiembre 1977, Akebonomaru, erróneamente identificado como $P$. brasiliensis. FAKU 117415, 1 (261 mm LS), Patagonia chilena, 5 septiembre 1977, Akebonomaru, erróneamente identificado como $P$. brasiliensis. FAKU 117418, 1 (262 mm LS), Patagonia chilena, 5 septiembre 1977, Akebonomaru, erróneamente identificado como $P$. brasiliensis. FAKU CP 189, 1 (257 mm LS), sin fecha de colecta. FAKU CP 628, 1 (224 mm LS), sin fecha de colecta. FAKU 124804, 1 (265 mm LS), Patagonia chilena, 40ํ12' S, 740ㅜㅇ' O, 1 septiembre 1977, erróneamente identificado como P. brasiliensis. FAKU 124797, $1(276 \mathrm{~mm}$ LS), Patagonia chilena, $40^{\circ} 12^{\prime}$ S, 74으의 $\mathrm{O}, 1$ septiembre, 1977, erróneamente identificado como P. brasiliensis. FAKU 125016, $1(238 \mathrm{~mm}$

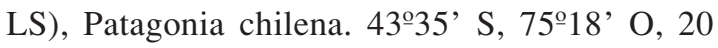
septiembre 1977, erróneamente identificado como Hippoglossina macrops Steindachner, 1876. FAKU 110474, 1 (309 mm LS), sin fecha de colecta. FAKU 111267, 1 (249 mm LS), sin fecha de colecta. FAKU 111265, 1 (195 mm LS), sin fecha de colecta.
Paralichthys oblongus (Mitchill, 1815): USNM 286117, 4 (154-195 mm LS), Océano Atlántico, costa este de Florida; 11 agosto 1974. USNM 286122, 4 (171-220 mm LS), $29^{\circ} 54^{\prime} \mathrm{N}-80^{\circ} 10^{\prime} \mathrm{O}$; 9 febrero 1965 . USNM 302461, 3 (196-221 mm LS), 40ํํ' ' S, 69ํ12' O, $183 \mathrm{~m}$. MNHN A 2428, 1 (193 mm SL), USA, Massachusetts, Woods Hole, 1880. MNHN 1896-0150, 1 (76 mm LS).

Paralichthys olivaceus (Temminck \& Schlegel, 1846): USNM 71996, 3 (158-186 mm LS), Otaru, Japón; 1906. FAKU 34344, 1 (436 mm LS), sin fecha de colecta.

Paralichthys orbignyanus MACN 793; 1; Playa Chica, Mar del Plata, Argentina; 1 agosto 1930. MACN 2802; 4 (30-293 mm LS); Río Quequén, Argentina; marzo 1928. MACN 2804; 3 (43-53 mm LT); Río Quequén; marzo 1928. MACN 6296; 1 (153 mm LS); San Clemente del Tuyú. MACN 6703; 1 (221 mm SL); Río Quequén; 25 enero 1963. MACN 4210; 2 (182, 194 mm LS); bahía San Blas; 25 enero 1952. MACN 6170; 2 (271, 275 mm LS); $F^{\circ}$ San Antonio, Provincia de Buenos Aires; MACN 7547; 5 (80-281 mm LS); San Antonio Oeste, Punta Villarino; 1937. USNM 77389; 1 (140 mm LS); Buenos Aires; 1888 (erróneamente identificado como $P$. brasiliensis). INIDEP 441; 2 (107, 160 mm LS); Laguna costera Mar Chiquita; 11 septiembre 1990. MNHN 1999-0295; 1 Sintipo (345 mm LS); Río de la Plata, Buenos Aires, Argentina, sintipo de Platessa orbignyana Valenciennes, 1839 In Voy. Amer. Merid., Atlas pl.16, fig. 1 et 1847 in Voy. Amer. Merid., Texte, 5, 2ème partie: POISSONS: 10. MCZ 11125; 2 (191, 400 mm LS); Rio Grande do Sul, 32 ${ }^{\circ} 03^{\prime} \mathrm{S}$ $52^{\circ} 08^{\prime} \mathrm{O} ; 8$ jun 1860. MCZ 11406; 1 (233 mm LS); Rio Grande do Sul; agosto 1865. 8 (133-204 mm LS); Santos, $23^{\circ} 56^{\prime} \mathrm{S}-4^{\circ} 22^{\prime} \mathrm{O}$; 1865. MCZ 11509; 1 (240 mm LS); Rio Grande, 32 ${ }^{\circ} 03^{\prime}$ S - 52 08' O; 8 jun 1860. MNRJ 5618; 1 (174 mm LS); Sec. Fed. de Pesca; Rio de Janeiro; abril 1949. MNRJ 8789; 1 (203 mm LS); Itacurussá, RJ; 1959. MNRJ 14360; 2 (173, 178 mm LS); Barra de Tramandaí, RS; 11 mayo 1977. MZUSP 27883; 1 (136 mm LS); Rio de Guarda, RJ; 27 julio 1983. MZUSP 14360; 2 (173, 178 mm LS); Barra de Tramandaí, 11 mayo 1977. INIDEP 445; 1 (400 mm LS); 34 $54^{\prime} \mathrm{S}, 55^{\circ} 35^{\prime} \mathrm{O}$; $11 \mathrm{~m}$; octubre 1992. MACN 1925; 1 (182 mm LS); Montevideo, Pescadería de Soriano Hnos. MACN 6780; 1 (281 mm LS); Montevideo; 1893. USNM 77388; 10 (126-174 mm LS); Montevideo 
(erróneamente identificado como $P$. brasiliensis). USNM 86733, 1 (212 mm LS), Río de la Plata, Montevideo, Albatross, 30 noviembre 1920 (erróneamente identificado como $P$. brasiliensis). MNHN 1999-0426, 3 (116-207 mm SL), Antillas, Martinique (erróneamente identificado como $P$. brasiliensis).

Paralichthys schmitti Ginsburg, 1933 (USNM 88831): holotipo; $374 \mathrm{~mm} \mathrm{LS;} \mathrm{Juan}$ Fernández; 17 diciembre 1927.

Paralichthys squamilentus Jordan \& Gilbert, 1882 (USNM 30862): holotipo; $98 \mathrm{~mm}$ LS; Pensacola, Florida, USA.

Paralichthys tropicus Ginsburg, 1933: MCZ 41042, 1 (258 mm LS), Venezuela, Sucre, 1 milla de Cumaná. USNM 34919, holotipo (259 mm LS), Trinidad 1037' S, 6142' O, 14 marzo 1966.

Paralichthys woolmani Jordan \& Williams, en Gilbert, 1897: USNM 47575, holotipo (196 mm LS), Islas Galápagos, Ecuador. USNM 82698, 1 (198 mm SL), Panamá. USNM 81634, 1 (114 mm SL), Panamá.

\section{Métodos}

Para la preparación de los esqueletos se siguió a Ossian (1970), Mayden \& Wiley (1984) y Díaz de Astarloa (1994). Las técnicas de clareado y tinción de tejidos óseos y cartilaginosos se efectuaron de acuerdo a las indicaciones de Dingerkus \& Uhler (1977), Potthoff (1984) y Kawamura \& Hosoya (1991). Las disecciones, observaciones y dibujos se realizaron macroscópicamente y con un microscopio estereoscópico marca Wild modelo M8, provisto de cámara clara. Para la nomenclatura de los huesos se siguió a Cervigón (1985), Rojo (1988) y Hoshino \& Amaoka (1998). Las abreviaturas usadas para los huesos fueron las que se describen en el Anexo 1.

\section{RESULTADOS Y DISCUSIÓN}

\section{Características generales del neurocráneo}

Kyle (1921) en su trabajo sobre la asimetría, metamorfosis y origen de los peces planos, realiza un estudio comparativo de varios géneros, tales como Rhombus, Hippoglossus, Pleuronectes y Solea. El autor establece una clasificación de tres principales grupos de acuerdo a las características asimétricas y estructura general de los cráneos: un primer grupo, constituido por formas grandes de elevado peso con huesos sólidos. Incluye en este grupo a Platysomatichthys, Hippoglossus, Pleuronectes y Rhombus. Un segundo grupo, formado por ejemplares delgados con huesos finos pero firmemente osificados. Los géneros Bothus, Arnoglossus y Citharus estarían comprendidos en este grupo. Por último, el tercer grupo estaría formado por formas elongadas con una osificación comparativamente más débil. Solea, Cynoglossus y Symphurus estarían comprendidos en este grupo.

El género Paralichthys incluye formas robustas, de elevado peso y con huesos sólidos bien osificados. De acuerdo a estas características podría incluirse a Paralichthys en la primera clasificación de Kyle (1921).

En líneas generales el neurocráneo de Paralichthys no presenta grandes diferencias con otros Teleósteos, excepto por la marcada asimetría manifestada principalmente en las regiones olfatoria y orbitaria, siendo los huesos pertenecientes a dichas regiones los más afectados por la torsión. Desde el margen posterior del neurocráneo hasta la fosa de articulación con el hiomandibular, la simetría de los huesos comprendidos en esa región se mantiene como en otros peces teleósteos. Sin embargo, desde ese punto hacia adelante se incrementa la asimetría de los huesos. La forma es más o menos rectangular, tanto en vista dorsal como ventral, con una baja cresta supraoccipital (Fig. 1). Si bien las especies de Paralichthys presentan en general una baja cresta (Woolcott et al. 1968, Díaz de Astarloa 1991), en $P$. orbignyanus es más alta que en las otras dos especies y termina posterodorsalmente en vértice agudo, mientras que en $P$. patagonicus y $P$. isosceles termina en forma redondeada. La relación longitud total/altura máxima del neurocráneo dio un valor medio mayor para $P$. isosceles que para las otras especies. Dicha altura cabe poco más de 3 veces en la longitud total en $P$. isosceles y 2,8 veces en $P$. orbignyanus. El diámetro horizontal de la órbita en relación al largo del neurocráneo, dio un valor mínimo de 1,14 para $P$. isosceles y un valor máximo de 2,4 para $P$. patagonicus. Esto indica que el ojo en $P$. isosceles es más grande que en $P$. patagonicus y $P$. orbignyanus, siendo en esta última especie proporcionalmente el más pequeño (Fig. 1). 
(A)
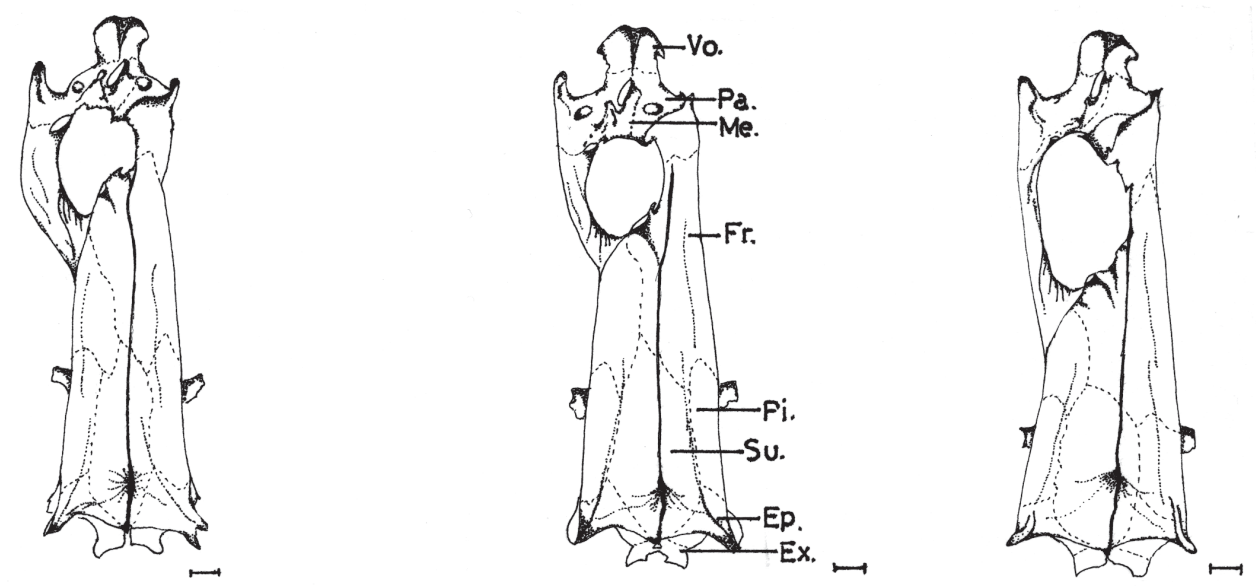

(B)
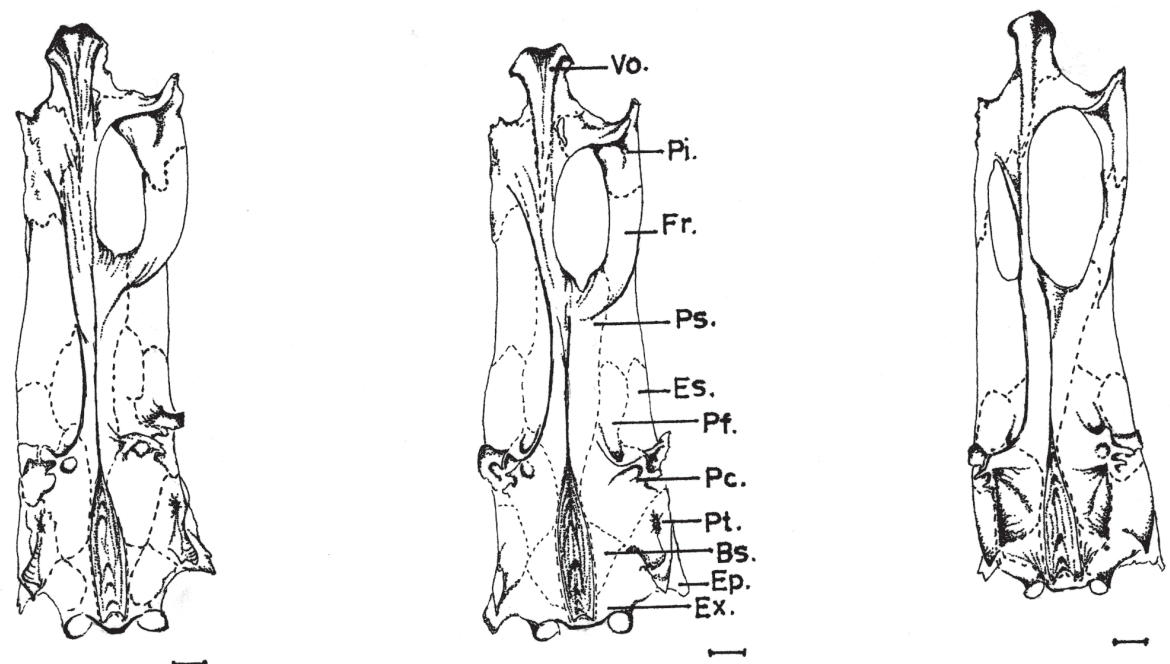

(C)
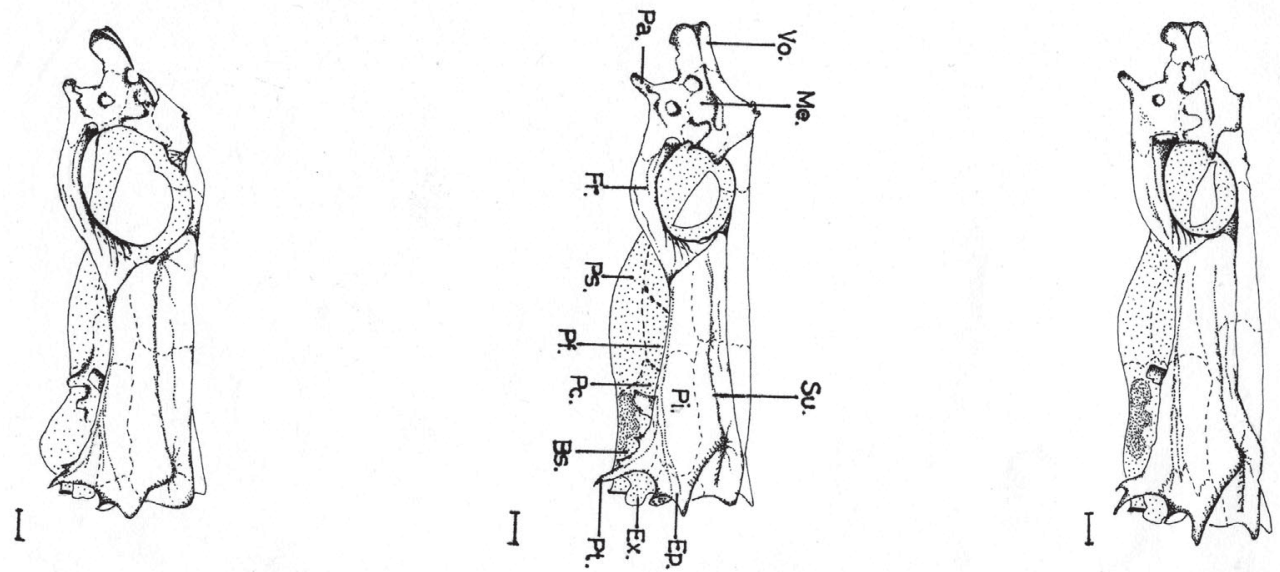

Fig. 1: Neurocráneos de Paralichthys orbignyanus (PO), P. patagonicus (PP) y P. isosceles (PI) en vistas dorsal (A), ventral (B) y lateral (C). Ver abreviaturas en el texto. Escala $1 \mathrm{~mm}$.

Neurocrania of Paralichthys orbignyanus (PO), P. patagonicus (PP) and P. isosceles (PI) in dorsal (A), ventral (B) and lateral views $(C)$. See abbreviations in text. Scale bar indicates $1 \mathrm{~mm}$. 


\section{Región olfatoria}

Consta de los siguientes huesos: mesetmoides y prevómer (impares); paretmoides y nasales (pares).

El mesetmoides está ubicado en la parte anterosuperior del neurocráneo. Debido a su origen como fusión de un hueso dérmico (rostrodermetmoides) y un elemento de cartílago (supraetmoides), en algunos trabajos también se lo denomina dermetmoides (Patterson 1976). Se caracteriza por tener una proyección aguzada dirigida hacia adelante, por encima y al costado de un borde medio dorsoventral. Díaz de Astarloa (1991) menciona erróneamente que dicho borde se prolonga inferiormente hasta suturarse con el prevómer en Paralichthys patagonicus. En realidad, tanto en esta especie como en $P$. isosceles y $P$. orbignyanus, la relación con el prevómer es a través de un cartílago etmoidal, no existiendo un contacto directo entre ambos huesos. Hacia los costados, el mesetmoides está articulado por sinartrosis dentada con los paretmoides (etmoides laterales). Junto al paretmoides derecho contribuye a formar el borde anterior de la órbita. Hacia la parte posterior y a través de una proyección está articulado con el frontal izquierdo (Fig. 1). Superficialmente, hacia arriba y al costado izquierdo está limitado con los huesos nasales. Por la parte lateral externa, del lado izquierdo, está dispuesto la porción anterior del canal sensorial supraorbitario izquierdo que, procedente del frontal, continúa por el hueso nasal y se prolonga por delante del mesetmoides desembocando en el borde lateral derecho para ponerse en contacto con la rama correspondiente del canal sensorial derecho. La proyección aguzada, en forma de gancho, que caracteriza al mesetmoides está más desarrollada en Paralichthys orbignyanus y $P$. patagonicus que en $P$. isosceles. Además en esta última especie, la cara externa de dicha proyección presenta una fuerte concavidad, ausente en las otras especies (Fig. 2).

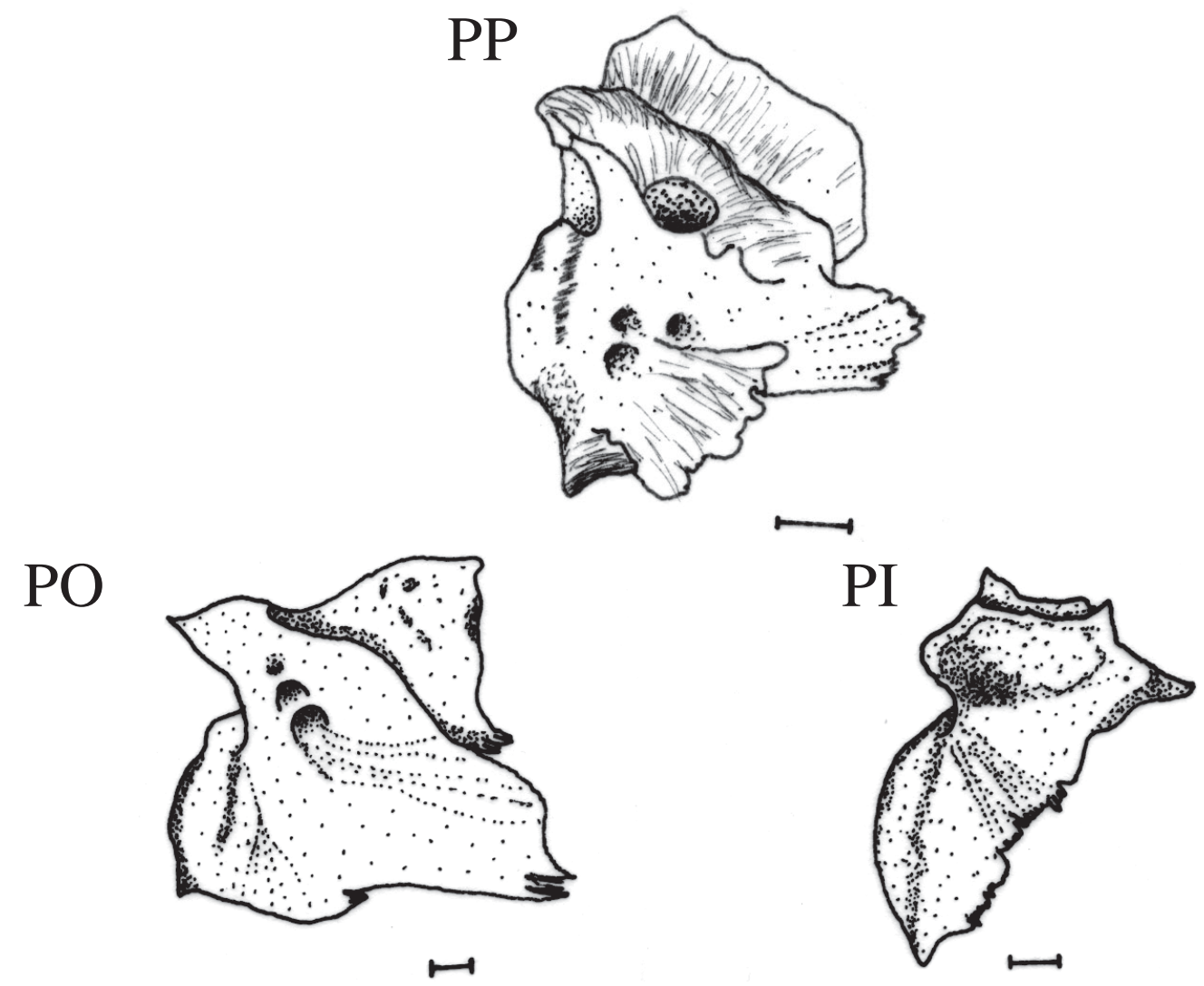

Fig. 2: Mesetmoides en vista lateral de Paralichthys patagonicus (PP), P. orbignyanus (PO) y $P$. isosceles (PI). Escala $1 \mathrm{~mm}$.

Mesethmoids of Paralichthys patagonicus (PP), P. orbignyanus (PO) and P. isosceles (PI) in lateral view. Scale bar indicates $1 \mathrm{~mm}$. 
El prevómer, hueso edéntulo y situado antero-inferiormente, acompaña el sentido de la torsión hacia el lado oculado y consecuentemente hay un desplazamiento anterior mayor del lado izquierdo. Esta torsión determina una asimetría entre el proceso izquierdo y el derecho de este hueso. De esta manera, el proceso izquierdo queda ubicado en una posición más o menos vertical, en la línea determinada por el eje medio del basioccipital y el segmento posterior del parasfenoides. Está articulado por sincondrosis con este último a través de la proyección espinosa posterior del prevómer que está inserta en una depresión ventral del extremo anterior del parasfenoides.
Dorsalmente, el cartílago etmovomerino pone en contacto el prevómer con la porción inferior del borde medio dorsoventral del mesetmoides. Posterolateralmente, sendas expansiones alares están suturadas con los paretmoides por los bordes anteroventrales de estos últimos (Fig. 1). No hay prácticamente diferencias morfológicas entre los huesos de las tres especies, excepto la presencia de una escotadura en la parte posterior y superior del prevómer bien desarrollada en $P$. orbignyanus y $P$. isosceles, y menos desarrollada en $P$. patagonicus (Fig. 3). Dicha escotadura o sínfisis delimita las proyecciones alares que están suturadas a los huesos paretmoides.
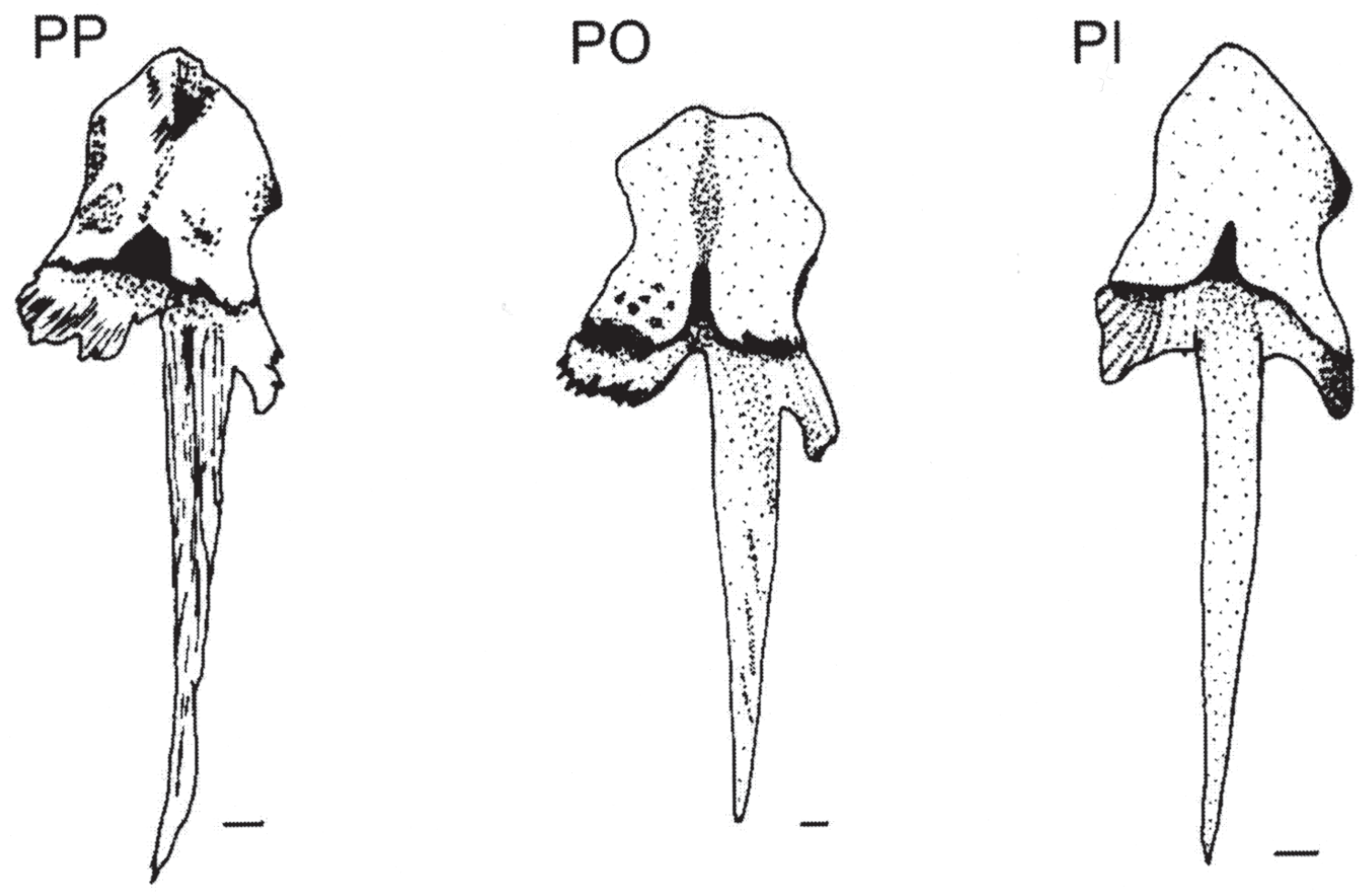

Fig. 3: Prevómeres en vista dorsal de Paralichthys patagonicus (PP), P. orbignyanus (PO) y $P$. isosceles (PI). Escala $1 \mathrm{~mm}$.

Prevomers of Paralichthys patagonicus (PP), P. orbignyanus (PO) and P. isosceles (PI) in dorsal view. Scale bar indicates $1 \mathrm{~mm}$. 
A los paretmoides también se los denomina etmoides laterales o pleuroetmoides (Hoshino \& Amaoka 1998). Algunos autores (Kyle 1921, Woolcott et al. 1968) los llaman prefrontales; sin embargo, el prefrontal es un hueso dérmico que pertenece a la serie circumorbitaria y que a veces se fusiona con el etmoides lateral formando un hueso de origen mixto (Rojo 1988), mientras que el paretmoides es de origen cartilaginoso. Los dos paretmoides (derecho e izquierdo) presentan una acusada asimetría (Fig. 4). El izquierdo está proyectado hacia adelante a través de un proceso espinoso anteroventral formando el margen anterior de la órbita izquierda. Posteriormente está suturado fuertemente con el frontal izquierdo. Por su borde anterodorsal está unido al mesetmoides, mientras que anteroventralmente está relacionado por sinartrosis con la proyección alar del prevómer. Woolcott et al. (1968) no observaron ninguna conexión entre el paretmoides izquierdo y el parasfenoides. En nuestro caso, la unión de los dos huesos se hizo manifiesta en las tres especies de Paralichthys estudiadas (Fig. 1B). El paretmoides derecho es un hueso grande con una expansión alar, laminar, que recorre el borde dorsolateral del mismo. Posteriormente presenta dos procesos espinosos (tres en $P$. isosceles). El superior está articulado por sinartrosis con el frontal derecho constituyendo junto con este el borde superior de la órbita, mientras que el inferior lo hace con el parasfenoides. Por el borde interno está suturado fuertemente con el mesetmoides y ventralmente con el prevómer. Cada paretmoides está perforado por un gran orificio que es el foramen olfatorio. El foramen es más pequeño en $P$. isosceles que en $P$. patagonicus y $P$. orbignyanus, tomando en cuenta ejemplares de la misma talla. En $P$. isosceles el proceso espinoso del paretmoides izquierdo está menos desarrollado que en las otras dos especies. Dicho proceso demarca en la cara interna del hueso una fosa, ausente en $P$. patagonicus y $P$. orbignyanus. Las distinciones entre los paretmoides derechos de las tres especies son aún mayores. El borde postero-superior del hueso presenta una concavidad en $P$. patagonicus, es convexo en $P$. isosceles y aserrado en $P$. orbignyanus. El foramen olfatorio está delimitado exteriormente por un repliegue óseo que en $P$. isosceles constituye una concavidad más profunda que en las otras dos especies.
Los nasales son pequeños huesos de canal (extensiones óseas del canal sensorial supraorbitario derecho e izquierdo) de forma cilíndrica. Están fuertemente adheridos a la piel y por consiguiente tienden a perderse durante la cocción. Woolcott et al. (1968) encuentran un solo nasal; sin embargo en el presente estudio se constató la presencia de ambos (Fig. 5). El nasal izquierdo se halla ubicado dorsalmente al foramen del paretmoides izquierdo. El derecho, y debido al proceso de torsión, toma una posición más central y se ubica en la parte superior del mesetmoides. Díaz de Astarloa (1991) menciona que en $P$. patagonicus estos huesecillos mostraron diversas formas; cilíndricas y piramidales. Los huesos descriptos de forma piramidal, en realidad corresponden al hueso lacrimal derecho, ubicado muy cerca del hueso nasal.

\section{Región orbitaria}

Esta región también se halla afectada por la torsión y consecuentemente los huesos que la componen presentan diferentes grados de asimetría. Todos los huesos presentes en esta región son pares. Los pterosfenoides son los únicos endocondrales. Los huesos escleróticos (anteriores y posteriores), frontales e infraorbitarios son de origen dérmico. El ojo derecho (superior) está implantado en una órbita ósea formada por los frontales, posteriormente y por los paretmoides y mesetmoides, anteriormente. El ojo izquierdo (inferior), en cambio, no queda encapsulado en órbita alguna sino que está situado directamente sobre el músculo aductor mandibular y sostenida por el endopterigoides subyacente.

A los infraorbitarios, en algunos trabajos, se los llama suborbitarios (Amaoka 1969). Sin embargo Cervigón (1980) aclara: "Se denominan suborbitarios a los huesos que se encuentran en la región de la mejilla en algunas formas primitivas como Chondrostei y Lepisosteus". Futch et al. (1972) mencionan como característica de Achirus lineatus (Linnaeus, 1758) (Soleidae) la ausencia de la serie de huesos infraorbitarios típica de la mayoría de los Teleósteos. Cervigón (1985) también afirma que normalmente no existen estos huesos en las especies de los géneros Achirus y Trinectes; no obstante el autor 


\section{Izquierdo}

PP

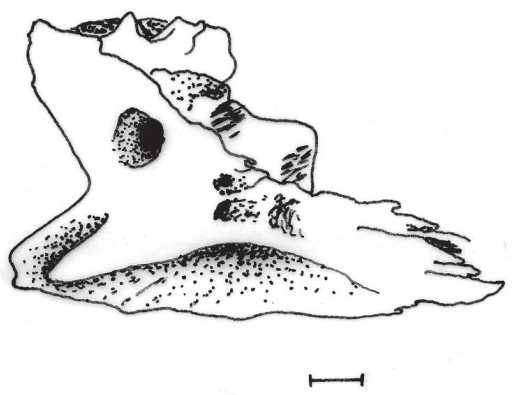

$\mathrm{PO}$

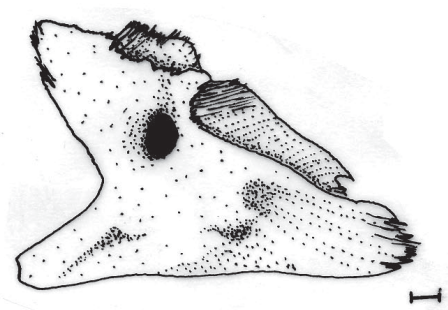

PI

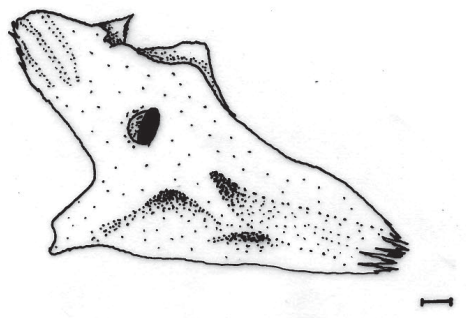

Derecho
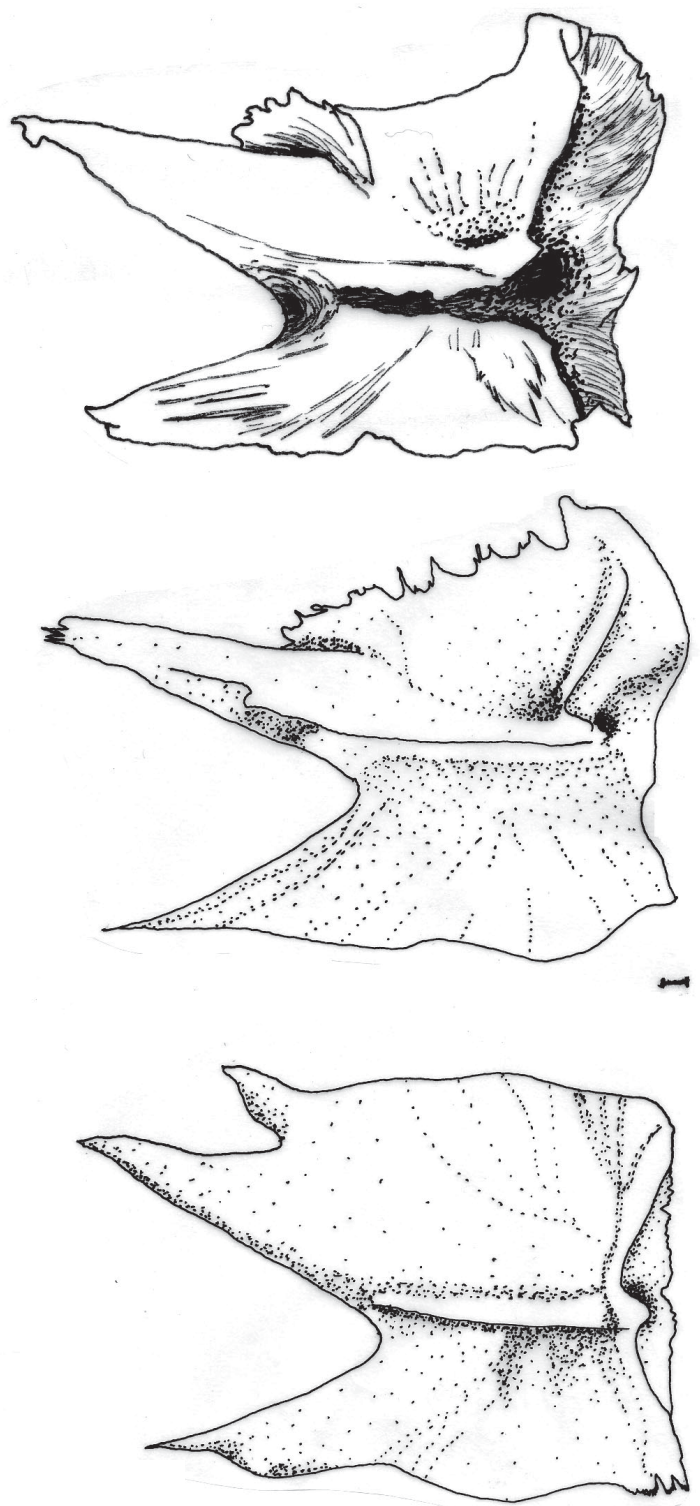

Fig. 4: Paretmoides izquierdo y derecho en vista lateral de Paralichthys patagonicus (PP), $P$. orbignyanus $(\mathrm{PO})$ y $P$. isosceles $(\mathrm{PI})$. Escala $1 \mathrm{~mm}$.

Left and right parethmoids of Paralichthys patagonicus (PP), P. orbignyanus (PO) and P. isosceles (PI) in lateral view. Scale bar indicates $1 \mathrm{~mm}$.

encuentra una serie de tres huesecillos en un ejemplar de T. paulistanus (Miranda Ribeiro, 1915) y en otro de A. lineatus, aclarando que posiblemente sean infraorbitarios. Woolcott et al. (1968) se refieren a la presencia de un solo hueso lacrimal de forma triangular débilmente adherido a la región etmoidal del lado oculado en el género Paralichthys, pero no mencionan otros de la serie. Amaoka (1969) encuentra una serie de huesos infraorbitarios presentes en la familia Paralichthyidae. También se hallan presentes en los siguientes grupos: Psettodidae, Scophthalmidae, Pleuronectinae, Achirinae, en los géneros Lepidoblepharon, Citharoides y Eucitharus de la familia Citharidae, y en los géneros Pseudorhombus y posiblemente 
Tephrinectes de la familia Paralichthyidae (Hensley \& Ahlstrom 1984). La presencia en Tephrinectes sinensis (Lacepède 1802) ha sido recientemente confirmada (Hoshino \& Amaoka 1998). Amaoka (1969) denomina al lacrimal como hueso preorbitario. Menciona que generalmente es más grande que cualquiera de los otros infraorbitarios y está rígidamente adherido al proceso lateral del paretmoides por un ligamento. Asimismo aclara que el preorbitario del lado ciego está bien desarrollado en Paralichthyidae, pero ausente en Bothidae. El lacrimal del lado izquierdo, de forma triangular, está unido ligeramente a la proyección anteroventral del paretmoides, superficialmente por encima de la protuberancia del cuello del maxilar y cubriendo en parte la cabeza del palatino (Fig. 6). Dicho hueso coincide con la descripción dada por Woolcott et al. (1968) para el lacrimal. Los autores, sin embargo, no mencionan el correspondiente al lado ciego. En este trabajo, dicho huesecillo presentó una forma piramidal, aplastado lateralmente. La forma es semejante a la observada en otra especie del género, Paralichthys olivaceus (Amaoka 1969). El lacrimal del lado ciego está unido a la proyección anterodorsal del etmoides lateral por una membrana cartilaginosa y cubre parcialmente el proceso palatino, pero no llega a superponerse al maxilar (Fig. 7). La serie infraorbitaria del lado oculado constituye un anillo óseo que bordea por detrás y por debajo al ojo inferior (Fig. 6). Estos huesos de canal sirven de vehículo a la rama infraorbitaria del sistema de la línea lateral que de un canal del hueso frontal está prolongada a través del lacrimal. En el lado ciego, la serie de huesos del canal sensorial se extiende anteriormente del hueso frontal derecho, recorre todo el borde lateral del paretmoides y concluye en el hueso lacrimal (Fig. 7). El número de huesos de la serie en el lado oculado es mayor en $P$. patagonicus $($ rango $=14-18$, media $=15.4$ ) que en $P$. orbignyanus $($ rango $=14-16$, media $=$ 14.7) y $P$. isosceles $($ rango $=12-15$, media $=$ 13.3). En el lado derecho, el número es superior en $P$. orbignyanus (6), siguiendo $P$. isosceles $($ rango $=4-6$, media $=5.14)$ y $P$. patagonicus $($ rango $=4-5$, media $=4.66$ ). Amaoka (1969) señala un número de cinco huesos más el preorbitario (lacrimal) en el lado ciego de $P$. olivaceus. Los huesos lacrimales en el lado oculado son morfológicamente diferentes en las tres especies. En $P$. orbignyanus y $P$. isosceles la longitud de la rama superior del hueso es la mitad más corta que la inferior, mientras que en $P$. patagonicus dicha longitud es ligeramente más corta, pero nunca la mitad. Las ramas superior e inferior son mucho más delgadas en $P$. isosceles, siendo más gruesas y robustas en $P$. patagonicus y $P$. orbignyanus. En el punto de confluencia de ambas ramas existe una profunda concavidad en $P$. isosceles, menos marcada en $P$. patagonicus y totalmente ausente en $P$. orbignyanus. Por último, la rama inferior describe un recorrido recto en $P$. patagonicus y $P$. orbignyanus, y curvo en $P$. isosceles (Fig. 8A). El hueso lacrimal correspondiente al lado ciego también es diferente de acuerdo a la especie de que se trate. En $P$. orbignyanus y $P$. patagonicus presenta una forma más o menos circular, aplastado lateralmente, mientras que en $P$. isosceles alcanza una forma piramidal a triangular, perforado por varios canales (Fig. 8B).

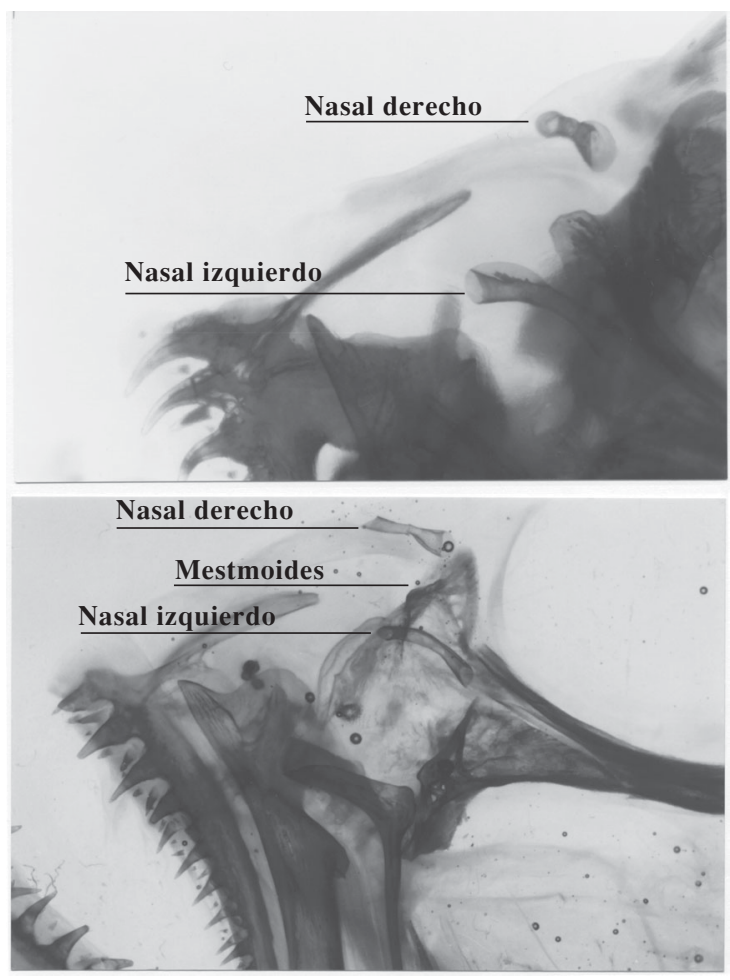

Fig. 5: Huesos nasales de Paralichthys patagonicus (arriba) y P. orbignyanus (abajo).

Nasal bones of Paralichthys patagonicus (upper) and $P$. orbignyanus (lower). 


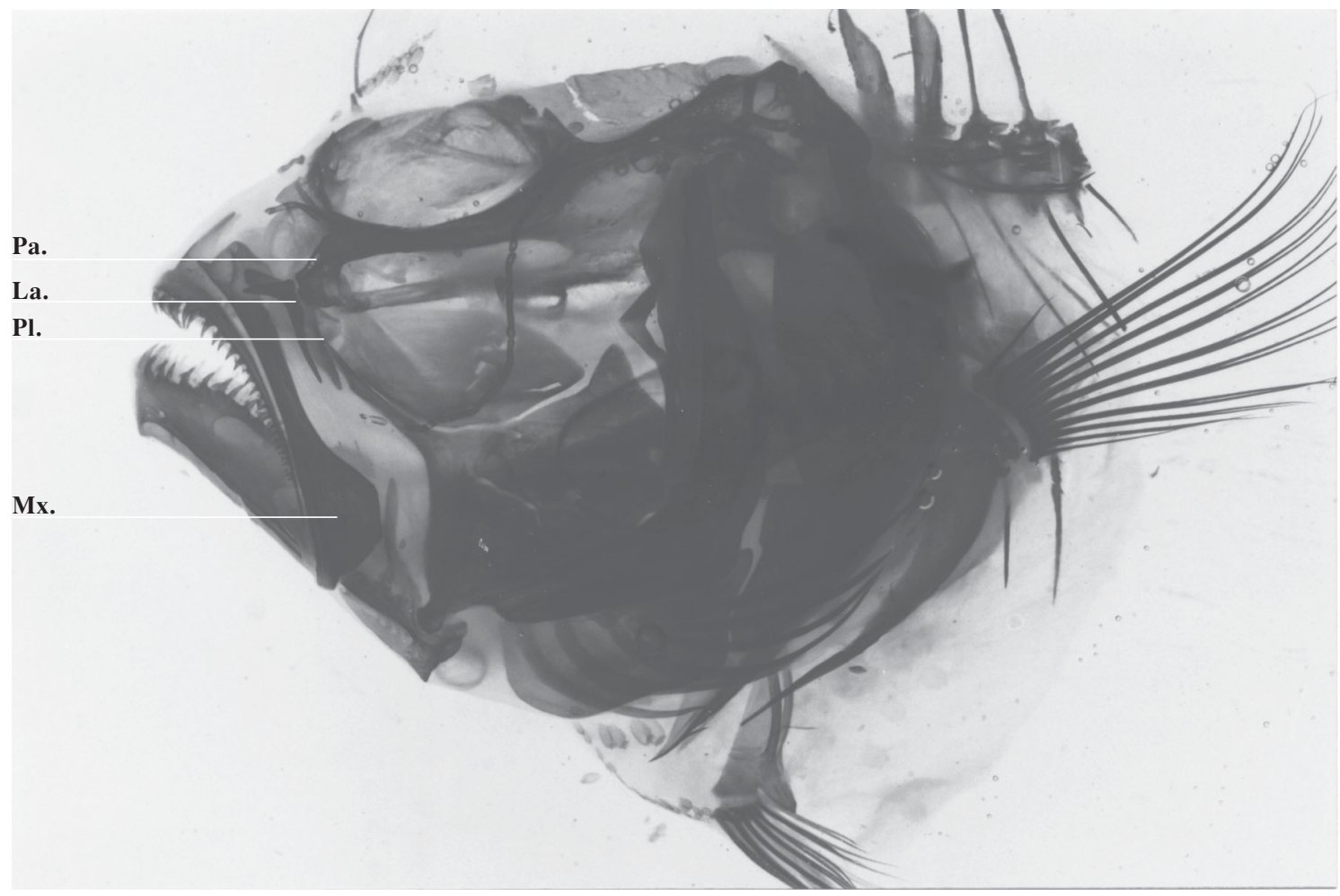

Fig. 6: Neuro y branquiocráneo de Paralichthys isosceles mostrando la serie de huesos infraorbitarios (I.or.) y el lacrimal (La.) del lado izquierdo. Ver otras abreviaturas en el texto.

Neurocranium and branchicranium of Paralichthys isosceles showing the infraorbital series of bones (I.or.) and lachrymal (La.) on left side. See other abbreviations on text.

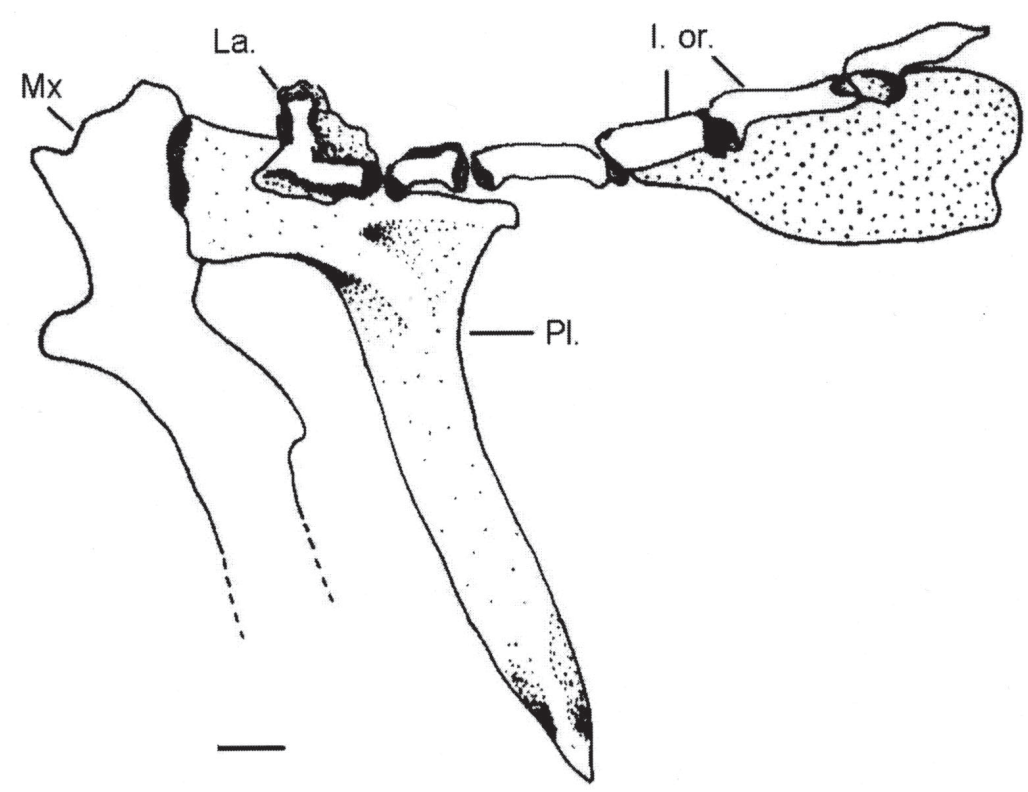

Fig. 7: Serie de huesos infraorbitarios (I.or) sobre el lado ciego de Paralichthys patagonicus. Ver otras abreviaturas en el texto. Escala $1 \mathrm{~mm}$.

Infraorbital bone-series (I.or.) on blind side of Paralichthys patagonicus. See other abbreviations on text. Scale bar indicates $1 \mathrm{~mm}$. 
(A)

PP
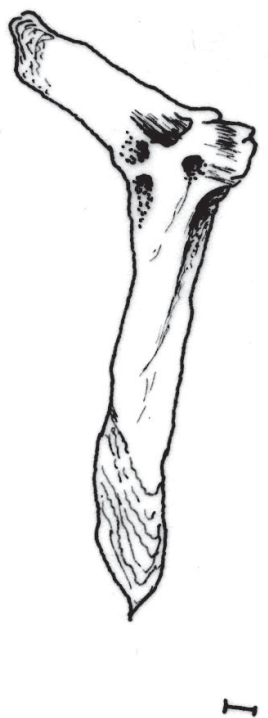

$\mathrm{PO}$

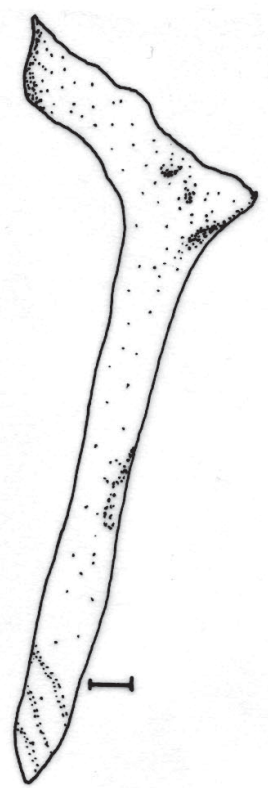

PI

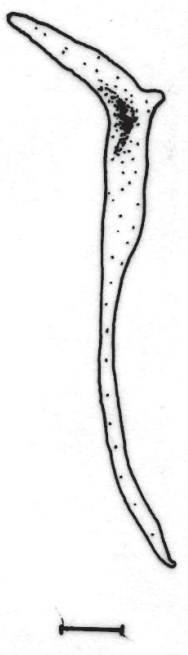

(B)
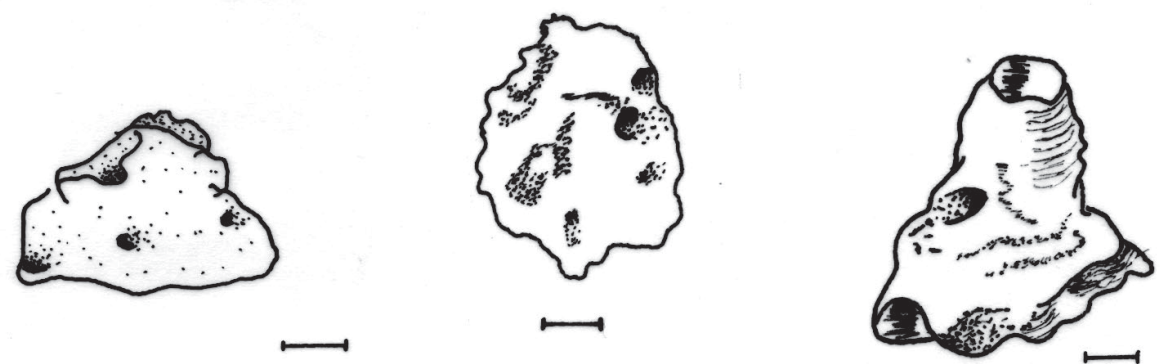

Fig. 8: Huesos lacrimales izquierdo (A) y derecho (B) de Paralichthys patagonicus (PP), $P$. orbignyanus $(\mathrm{PO})$ y $P$. isosceles $(\mathrm{PI})$ en vista lateral. Escala $1 \mathrm{~mm}$.

Left (A) and right (B) lachrymal bones of Paralichthys patagonicus (PP), P. orbignyanus (PO) and P. isosceles (PI) in lateral view. Scale bar indicates $1 \mathrm{~mm}$.

Los frontales contribuyen a formar los márgenes dorsoposterior, posterior e inferior de la órbita. El frontal izquierdo (Fig. 9, izquierda) ha sufrido un desplazamiento ventral y una torsión a lo largo de su eje longitudinal, que se hace más notoria en su parte anterior. Presenta una cresta en su parte media dirigida verticalmente, que se continúa con una cresta oblicua del frontal derecho. Ambas forman el borde posterior de la órbita. Anteriormente, el hueso está articulado por sinartrosis con el paretmoides izquierdo y con el mesetmoides. Hacia atrás y dorsalmente está suturado con el supraoccipital y parietal. Su borde posterolateral está relacionado con el pterótico, esfenótico, pterosfenoides y parasfenoides, a través de sinartrosis dentadas (Fig.1). Un sistema de canales atraviesan el hueso, a través de los cuales pasa la rama izquierda del sistema laterosensorial proveniente del pterótico, con 
que es continuado en el mesetmoides y la serie infraorbitaria. El frontal derecho constituye aproximadamente la mitad del margen dorsal de la órbita (Fig. 9, derecha). Presenta una proyección ventral curvada que se adelgaza a la mitad de su recorrido, para luego ensancharse anterodorsalmente en un pequeño proceso lateral bífido. Dicha proyección circunda interiormente el lado izquierdo de la órbita apoyándose en el frontal de ese mismo lado y conectándose hacia adelante con el mesetmoides. Tres crestas caracterizan al frontal derecho. Una en sentido oblicuo que, junto a la del frontal izquierdo, conforman el margen dorsoposterior y posterior de la órbita. Una segunda junto a la cresta del supraoccipital, constituyen la cresta medio dorsal del neurocráneo. La tercera constituye, junto al parietal y pterótico, el borde lateral derecho de la caja craneana. Hacia adelante, el frontal derecho está suturado fuertemente al paretmoides derecho. Hacia atrás y dorsalmente está relacionado con el supraoccipital y parietal. Ventralmente está suturado con el pterótico, esfenótico, pterosfenoides y parasfenoides. Todas las articulaciones son por sinartrosis dentadas. Los huesos frontales izquierdos presentan diferencias morfológicas entre las tres especies de Paralichthys. La rama o brazo anterior que se sutura al paretmoides es más ancha en $P$. orbignyanus mientras la menor anchura corresponde a $P$. isosceles. La longitud del brazo anterior, en relación al largo total del hueso, es un $55 \%$ en $P$. orbignyanus, $66 \%$ en $P$. patagonicus y $75 \%$ en $P$. isosceles. Esta relación está referida al mayor tamaño del ojo en $P$. isosceles respecto a las otras dos especies (ver Fig. 1). La cara interna del frontal derecho, que limita con el parasfenoides, presenta un borde saliente en $P$. orbignyanus y $P$. patagonicus, estando ausente en $P$. isosceles. En esta última especie el frontal derecho no está suturado con el parasfenoides.

Los pterosfenoides quedan delimitados por los frontales anteriormente, el parasfenoides por debajo, los esfenóticos por encima y hacia atrás por los proóticos (Fig. 1C). En ejemplares juveniles son difíciles de observar y se hallan incompletamente osificados (Woolcott et al. 1968). Los autores denominan en su trabajo a estos huesos como alisfenoides. Rojo (1988) recomienda rechazar este nombre ya que este hueso no es homólogo al alisfenoides del cráneo de los mamíferos. En Paralichthys patagonicus y $P$. orbignyanus ambos pterosfenoides son ligeramente asimétricos entre sí, siendo el derecho un poco más grande (Fig. 10A y 10B). En $P$. isosceles, en cambio, son marcadamente asimétricos. El izquierdo es más o menos circular y comprimido. El derecho presenta una expansión membranosa anterior que da al hueso una forma más o menos rectangular (Fig. 10C).

Los huesos escleróticos consisten en dos segmentos semicirculares (anterior y posterior) conectados por cartílago en la superficie lateral interna y por membranas corneales en la cara externa. Cada ojo presenta un par de estos segmentos. No se encontraron diferencias morfológicas entre los huesos de las tres especies.

\section{Región otico-occipital}

Los huesos de esta región forman la bóveda craneana y cápsula ótica. Proveen protección al cerebro, nervios craneales y sistema de equilibrio, y dan soporte al branquiocráneo, columna vertebral y cintura pectoral. La región está compuesta por huesos de origen cartilaginoso tanto pares (esfenóticos, pteróticos, proóticos, epioccipitales, intercalares y exoccipitales) como huesos impares (supraoccipital y basioccipital), y elementos dérmicos pares (parietales, postemporales y extraescapulares) e impares (parasfenoides).

Los esfenóticos no sufren una notable modificación por la torsión, siendo el derecho e izquierdo aproximadamente simétricos entre sí. Anterodorsalmente se unen a los frontales y posterodorsalmente a los pteróticos. Ventralmente están articulados por sinartrosis a los pterosfenoides, y un poco más hacia atrás con los proóticos con quienes comparten la formación de la fosa de articulación del proceso anterior del hiomandibular (Fig. 1B). Por encima de esta concavidad, un surco, también compartido con el pterótico, sirve de punto de origen de un importante músculo facial: el elevador del arco palatino. El proceso membranoso anterior que está articulado con el frontal es más corto y ancho en $P$. isosceles que en $P$. patagonicus y $P$. orbignyanus (Fig. 11C).

Los pteróticos son huesos grandes, más o menos simétricos, siendo el derecho ligeramente más grande que el izquierdo. Cada uno de ellos presenta una proyección curva 
digitiforme anterior que junto al parietal, que está inmediatamente por encima, forma el borde posterolateral del neurocráneo. El extremo de dicho proceso está suturado con el frontal. En su borde posteroinferior cada pterótico está suturado con el exoccipital. Siguiendo hacia adelante está articulado por sinartrosis con el borde posterodorsal del proótico y anteriormente con el borde posterior del esfenótico. Apoyados inmediatamente por encima se sitúan, el parietal hacia adelante y el epioccipital por detrás (Fig. 1B). El pterótico presenta tres orificios de comunicación del canal sensorial: el anterior para el canal temporal, el inferior para la rama preopérculomandibular, y el superior para el hueso extraescapular. La longitud del proceso anterior en relación al largo total del hueso es de un $44 \%$ en $P$. orbignyanus, un $40 \%$ en $P$. patagonicus y un $31 \%$ en $P$. isosceles. Las mismas proporciones se mantuvieron tanto para el hueso izquierdo como derecho (Fig. 12).

\section{Izquierda}

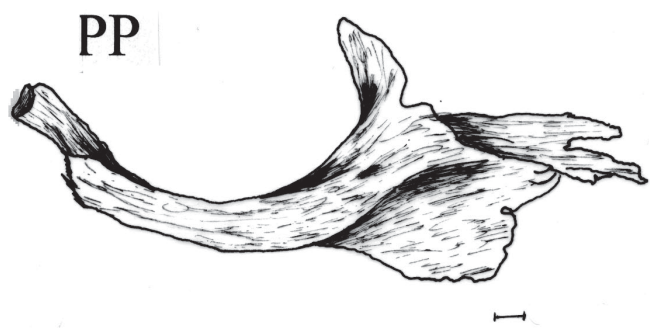

PO

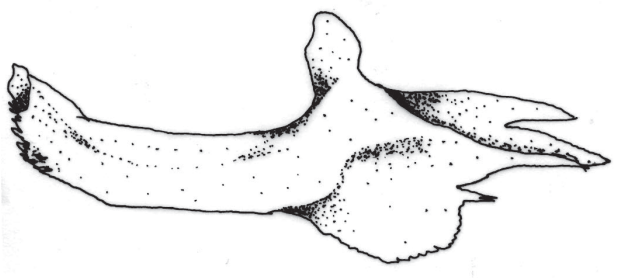

$\mapsto$

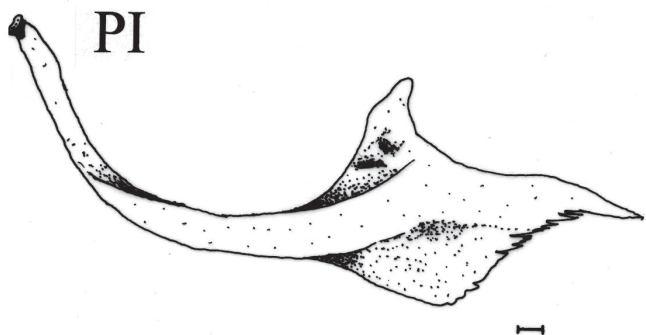

Derecha
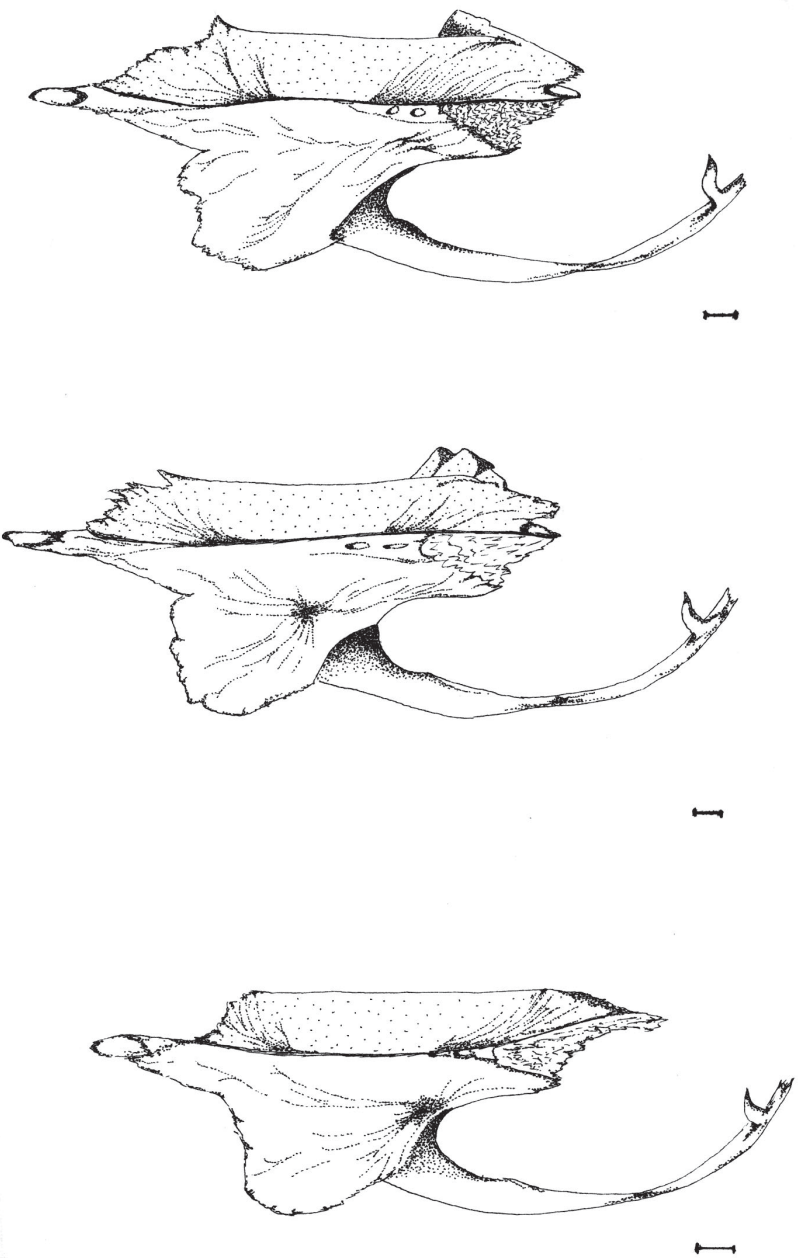

Fig. 9: Frontales izquierdo y derecho de Paralichthys patagonicus (PP), P. orbignyanus (PO) y $P$. isosceles (PI) en vista lateral. Escala $1 \mathrm{~mm}$.

Left and right frontals of Paralichthys patagonicus (PP), P. orbignyanus (PO) and P. isosceles (PI) in lateral view. Scale bar indicates $1 \mathrm{~mm}$. 

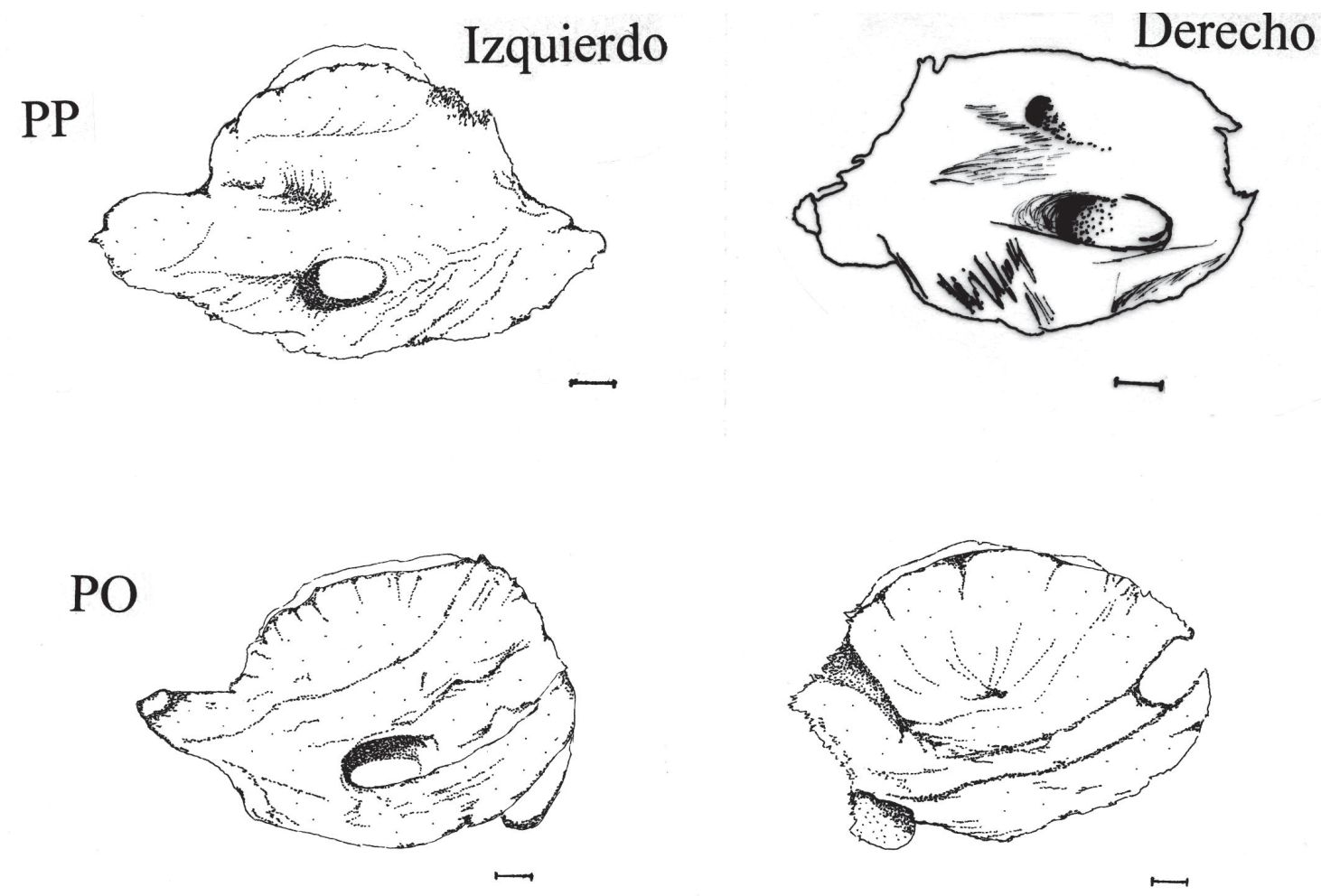

PI
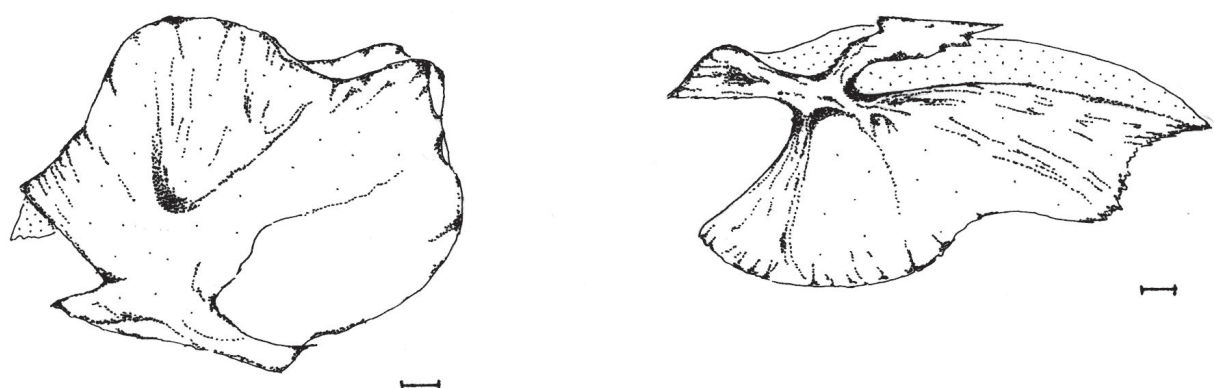

Fig. 10: Pterosfenoides izquierdo y derecho de Paralichthys patagonicus (PP), P. orbignyanus (PO) y $P$. isosceles $(\mathrm{PI})$ en vista lateral. Escala $1 \mathrm{~mm}$.

Left and right Pterosphenoids of Paralichthys patagonicus (PP), P. orbignyanus (PO) and P. isosceles (PI) in lateral view. Scale bar indicates $1 \mathrm{~mm}$. 

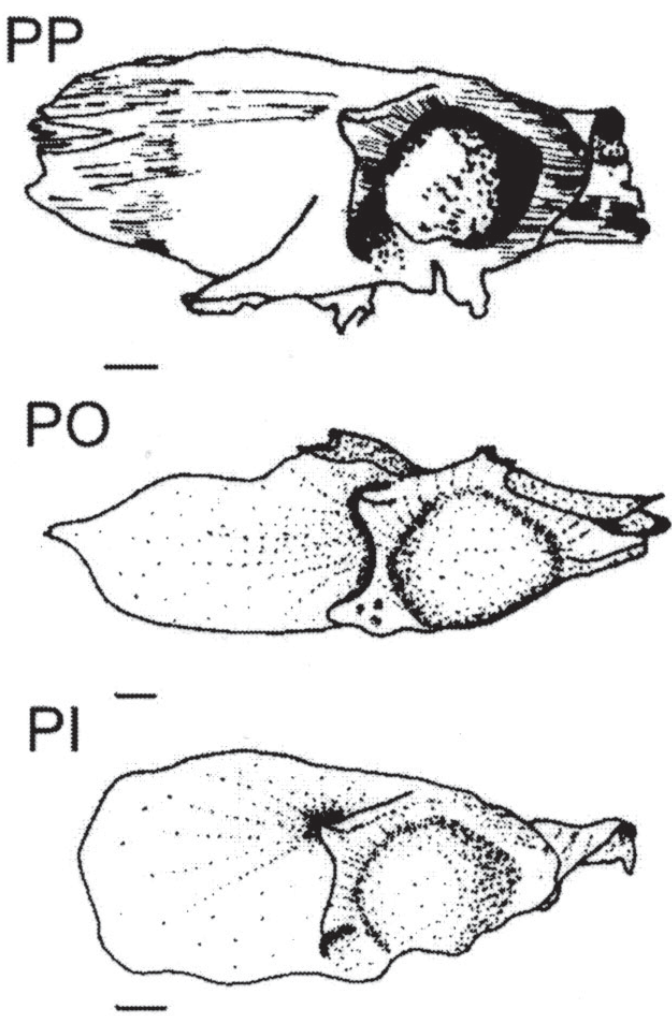

Fig. 11: Esfenóticos derechos de Paralichthys patagonicus (PP), $P$. orbignyanus (PO) y $P$. isosceles $(\mathrm{PI})$ en vista lateral. Escala $1 \mathrm{~mm}$.

Right sphenotics of Paralichthys patagonicus (PP), $P$. orbignyanus (PO) and $P$. isosceles (PI) in lateral view. Scale bar indicates $1 \mathrm{~mm}$.

Los proóticos son los huesos más grandes de la serie ótica. De forma más o menos cuadrangular, en su parte anterodorsal constituyen la otra mitad de la fosa hiomandibular, que comparten con los esfenóticos a los cuales están suturados. La superficie posterior de cada proótico es convexa y delgada; está suturada con el borde anterior, también convexo y delgado, del basioccipital, formando con este último la concavidad donde están alojados los otolitos sagitta. Ventralmente están articulados por sinartrosis con el parasfenoides. Con este último delimitan un foramen para el paso de las carótidas internas. Hacia adelante se ponen en contacto con el pterosfenoides. Por el borde posterodorsal están suturados con el pterótico (Fig. 1). Dorsoanteriormente el proótico está perforado por un foramen que da paso a la cámara
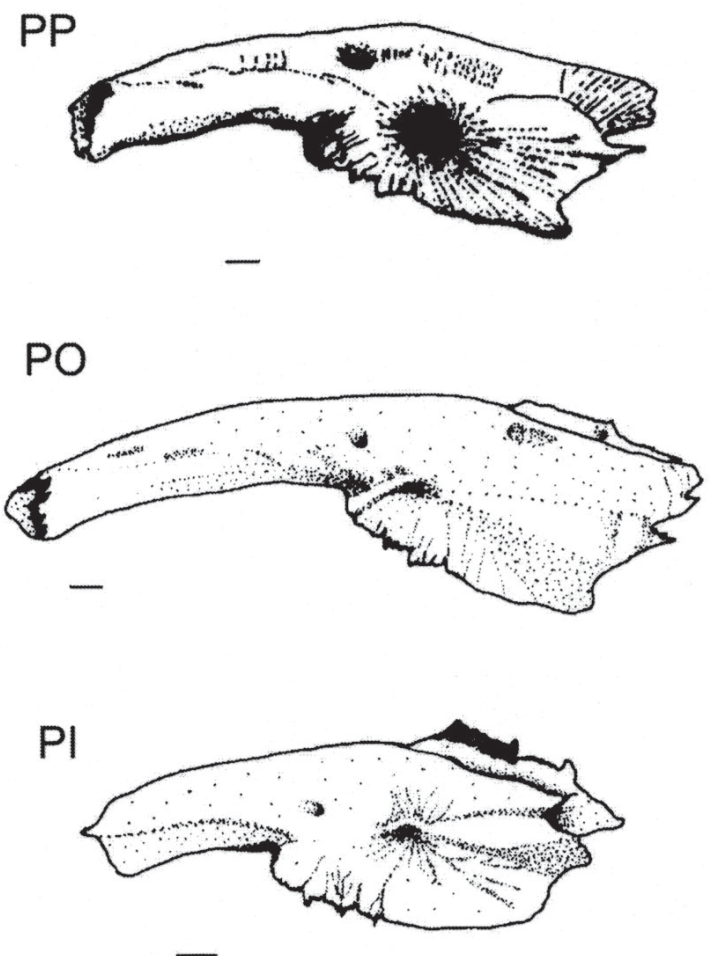

Fig. 12: Pteróticos derechos de Paralichthys patagonicus $(\mathrm{PP}), P$. orbignyanus $(\mathrm{PO})$ y $P$. isosceles (PI) en vista lateral. Escala $1 \mathrm{~mm}$.

Right pterotics of Paralichthys patagonicus (PP), $P$. orbignyanus (PO) and P. isosceles (PI) in lateral view. Scale bar indicates $1 \mathrm{~mm}$.

trigéminofacial (Topp \& Cole 1968). No se han observado diferencias de tamaño entre los huesos del lado izquierdo y derecho de las tres especies estudiadas. En Paralichthys patagonicus y $P$. orbignyanus, el foramen que da paso a la cámara trigéminofacial, se encuentra subdividido internamente por un puente óseo constituyendo dos pequeños orificios a través de los cuales pasan la vena yugular y la arteria orbital. Dicho foramen, en $P$. isosceles, se encuentra subdividido por un puente óseo incompleto o ausente (Fig. 13C). En la superficie de sutura del proótico con el esfenótico se constituye la fosa de articulación del hiomandibular. En ese punto el proótico presenta un proceso espinoso bien desarrollado que está proyectado verticalmente en $P$. patagonicus y $P$. isosceles, pero poco conspicuo, y a veces ausente, en $P$. orbignyanus (Fig. 13). 


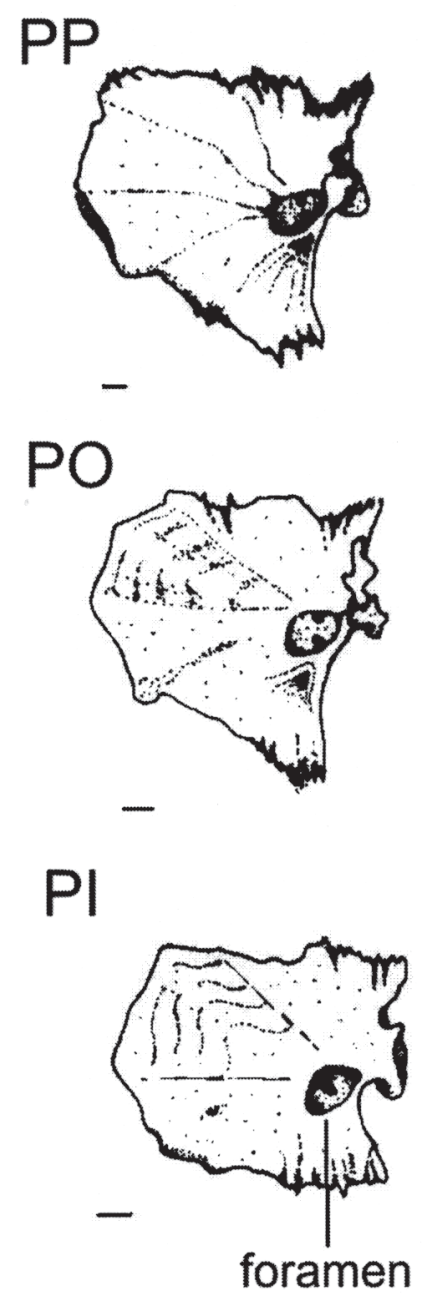

Fig. 13: Proóticos izquierdos de Paralichthys patagonicus (PP), P. orbignyanus (PO) y $P$. isosceles $(\mathrm{PI})$ en vista lateral. Escala $1 \mathrm{~mm}$.

Left prootics of Paralichthys patagonicus (PP), $P$. orbignyanus (PO) and $P$. isosceles (PI) in lateral view. Scale bar indicates $1 \mathrm{~mm}$.

La morfología del otolito sagitta ha sido intensamente estudiada como medio para la identificación de los restos de peces fósiles (Bauzá-Rullán 1957), para la estimación de la edad (Casselman 1983, Fabré \& Cousseau 1990) y en taxonomía (Bauzá-Rullán 1956, Fabré 1988, Vilela 1988, Aloisi 1990, Volpedo \& Echeverría 1997). En las tres especies de Paralichthys estudiadas, los otolitos sagitta, derecho e izquierdo, son asimétricos entre sí. El otolito izquierdo es de forma subtriangular con rostro anguloso y romo en $P$. patagonicus. En $P$. orbignyanus y $P$. isosceles es más o menos cuadrangular con rostro anguloso y redondeado en $P$. orbignyanus y prominente y redondeado en $P$. isosceles. La cisura es marcada en las tres especies, pero poco hendida en $P$. patagonicus y $P$. isosceles. La cara interna es levemente convexa en $P$. patagonicus y $P$. isosceles, pero con surco bien profundo en la primera especie. La cara interna es bien convexa y con un profundo surco en $P$. orbignyanus. El ostium es abierto en las tres especies. Alrededor del surco hay una depresión en forma de $\mathrm{V}$ menos profunda, presente en $P$. patagonicus y $P$. isosceles. No se observa dicha depresión en $P$. orbignyanus. El borde posterior muestra una escotadura en la parte media en $P$. orbignyanus y $P$. isosceles, siendo menos marcada en esta última especie. En $P$. patagonicus, la mencionada escotadura está corrida hacia el borde dorsal, delimitando un proceso más o menos cuadrangular. El borde dorsal es irregular, con una elevación media en $P$. orbignyanus, ausente en $P$. patagonicus y $P$. isosceles. El borde ventral es liso en $P$. patagonicus y $P$. isosceles; irregular y a veces liso en $P$. orbignyanus. La cara externa es cóncava y lisa en las tres especies (Fig. 14, izquierda).

El otolito derecho es de forma subtriangular en $P$. patagonicus, oblongo y a veces subtriangular en $P$. orbignyanus, y aproximadamente cuadrangular en $P$. isosceles. Rostro anguloso y romo con cisura marcada y levemente hendida en $P$. orbignyanus y $P$. isosceles. En $P$. orbignyanus la cisura está profundamente hendida. La cara interna es levemente convexa en $P$. patagonicus y $P$. isosceles, si bien $P$. patagonicus presenta un surco más profundo. En $P$. orbignyanus es bien convexa y con surco profundo. El ostium es abierto anteriormente en las tres especies. $\mathrm{Al}$ igual que en el otolito izquierdo, se manifiesta una depresión en forma de $\mathrm{V}$ alrededor del surco en $P$. patagonicus y $P$. isosceles, pero no en $P$. orbignyanus. El borde posterior presenta una escotadura media en $P$. patagonicus y $P$. orbignyanus, y apenas manifiesta en $P$. isosceles. El borde dorsal es irregular y con una elevación media en $P$. orbignyanus; es también irregular, pero sin dicha elevación en las otras dos especies. El borde ventral es liso en $P$. patagonicus y $P$. isosceles; festoneado o irregular en $P$. orbignyanus. La cara externa es cóncava y lisa en las tres especies de Paralichthys (Fig. 14, derecha). 

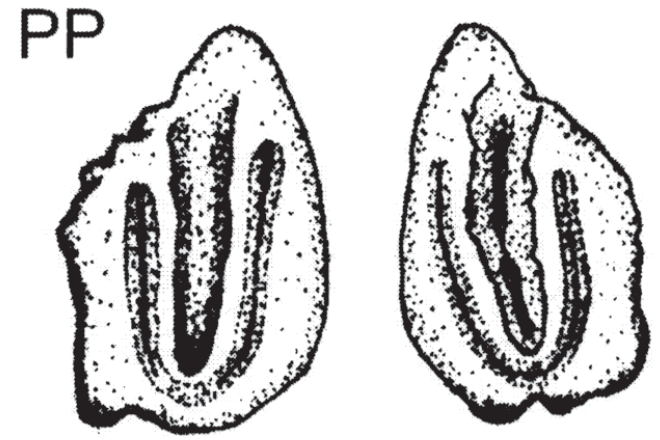

$\mathrm{PO}$
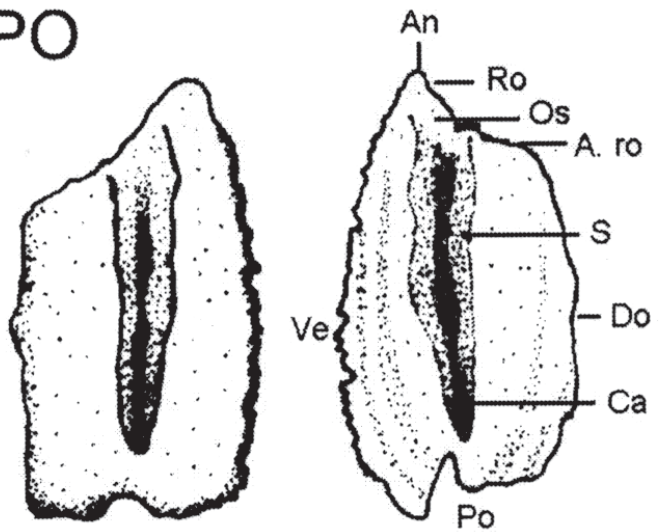

izquierdo
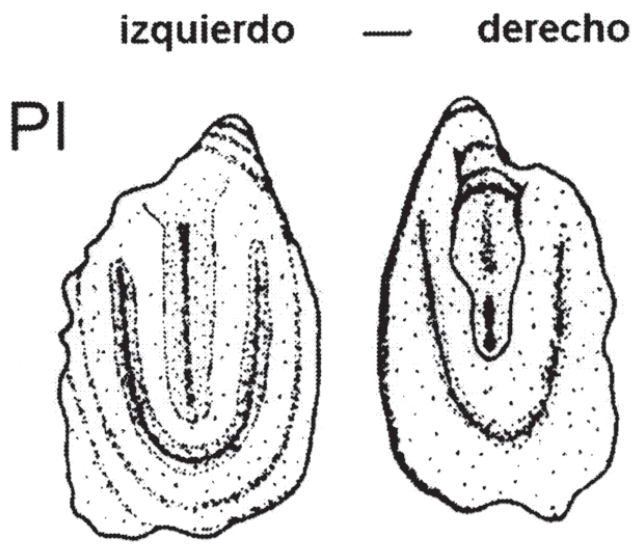

Fig. 14: Otolitos sagittae de Paralichthys patagonicus (PP), P. orbignyanus (PO) y $P$. isosceles (PI) en vista lateral. An: borde anterior; Ca: cauda; Do: margen dorsal; Os: ostium; Po: borde posterior; Ro: rostrum; S: sulcus; Ve: margen ventral. Escala $1 \mathrm{~mm}$.

Sagittae otoliths of Paralichthys patagonicus (PP), $P$ orbignyanus $(\mathrm{PO})$ and $P$. isosceles $(\mathrm{PI})$ in lateral view. An: anterior edge; Ca: cauda; Do: dorsal margin; Os: ostium; Po: posterior edge; Ro: rostrum; S: sulcus; Ve: ventral margin. Scale bar indicates $1 \mathrm{~mm}$.
Existen además diferencias morfométricas en los otolitos sagittae de las tres especies, como lo demuestran los trabajos realizados por Fabré (1988) para $P$. isosceles y Volpedo \& Echeverría (1997) para $P$. patagonicus y $P$. orbignyanus.

Los epioccipitales también se denominan epióticos. Según Patterson (1975) el epioccipital es una osificación de la región occipital que ha invadido la región ótica. Propone, por lo tanto, el término epioccipital para reemplazar el comúnmente utilizado de epiótico. Estos huesos constituyen el extremo posterodorsal de la caja craneana. Prácticamente no son afectados por el proceso de torsión. Se caracterizan por la presencia de dos procesos posterolaterales que delimitan una concavidad que sirve de inserción al extremo dorsal del postemporal. Hacia adelante están articulados por sinartrosis con el parietal; por el borde lateral interno lo hacen con el supraoccipital y hacia abajo con el pterótico; inferoposteriormente están suturados con el exoccipital (Fig. 1). Superficialmente el epioccipital está relacionado con la rama vertical del lateral extraescapular y con el dorsal extraescapular, de forma tubular. Los dos extraescapulares se hallan fuertemente adheridos a la piel, por lo que no hay sutura alguna con el epioccipital. No hubieron diferencias morfológicas entre los huesos de las tres especies de Paralichthys (Fig. 15).

Los intercalares son los huesos más pequeños de la serie ótica, pudiendo pasar inadvertidos cuando se realiza la desarticulación del neurocráneo. Woolcott et al. (1968) y Pérez \& Werner (1986) no describen estos huesos, probablemente debido a esta última característica. Están ubicados, a modo de cuña, entre la superficie posterior del pterótico y el borde anterior del exoccipital. Sobre ellos se inserta el ligamento del brazo inferior del postemporal. En las tres especies estudiadas la forma del hueso resultó ser irregular, no encontrándose características distintivas que permitan diferenciar a las tres especies, posiblemente debido a su morfología (Fig. 16).

Los exoccipitales están ubicados en la cara posterior de la caja craneana, por debajo de los epioccipitales. Hacia abajo están suturados fuertemente con el basioccipital con el que forman la articulación tricondilar con el atlas. Hacia arriba están suturados internamente entre 
sí, delimitando las paredes del foramen magnum. Anteriormente están articulados por sinartrosis con el pterótico y entre ellos, el diminuto intercalar. Dos prominentes forámenes atraviesan el cuerpo de cada exoccipital: el superior, permite el paso del 9o nervio craneal (glosofaríngeo) y el inferior lo hace para el $10^{\circ}$ nervio craneal (vago). Los dos exoccipitales son simétricos. Las diferencias de los huesos en las tres especies están dadas principalmente en el tamaño de los cóndilos occipitales. En $P$. isosceles constituyen una superficie de articulación menor que en $P$. patagonicus y orbignyanus (Fig. 17B). La osificación del hueso es otro carácter distintivo. En ejemplares de la misma talla, Paralichthys isosceles presenta una débil osificación (huesos más delgados y quebradizos) que en las otras dos especies del género. La forma del hueso, visto lateralmente, es más o menos piramidal, con una superficie de articulación espinosa en $P$. isosceles y menos manifiesta en $P$. patagonicus y $P$. orbignyanus.
El supraoccipital cubre la superficie dorsoposterior del cráneo. Visto desde arriba, es de forma romboidal con una cresta mediodorsal, que junto a la correspondiente cresta del hueso frontal derecho, conforman la quilla frontosupraoccipital del neurocráneo. Hacia adelante está articulado por sinartrosis con los frontales, a los costados con los parietales, posterolateralmente con los epioccipitales y posteriormente se pone en contacto con los exoccipitales (Fig. 1). Su superficie plana sirve de inserción a la porción anterior de la musculatura epaxial del cuerpo. En $P$. patagonicus y $P$. orbignyanus, la cresta supraoccipital es más baja que en $P$. isosceles. En esta última especie, la parte basal del supraoccipital es más corta que la cresta supraoccipital, mientras que en las otras dos especies la longitud es la misma (Fig. 18C). La cresta supraoccipital se ubica en la línea media del hueso en $P$. orbignyanus, mientras que en $P$. patagonicus se halla desplazada ligeramente hacia la derecha. En $P$. isosceles, ese
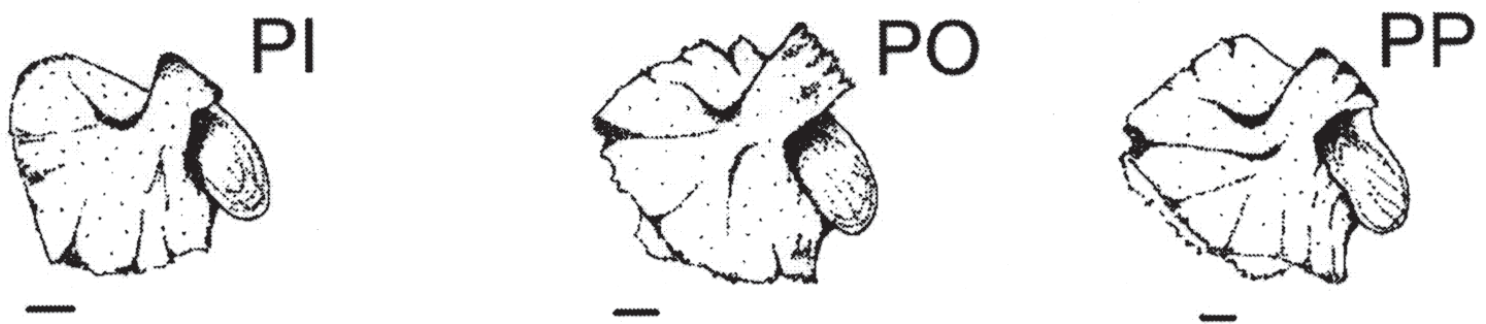

Fig. 15: Epioccipitales de Paralichthys patagonicus (PP), P. orbignyanus (PO) y P. isosceles (PI) en vista lateral. Escala $1 \mathrm{~mm}$.

Epioccipitals of Paralichthys patagonicus (PP), P. orbignyanus (PO) and P. isosceles (PI) in lateral view. Scale bar indicates $1 \mathrm{~mm}$.
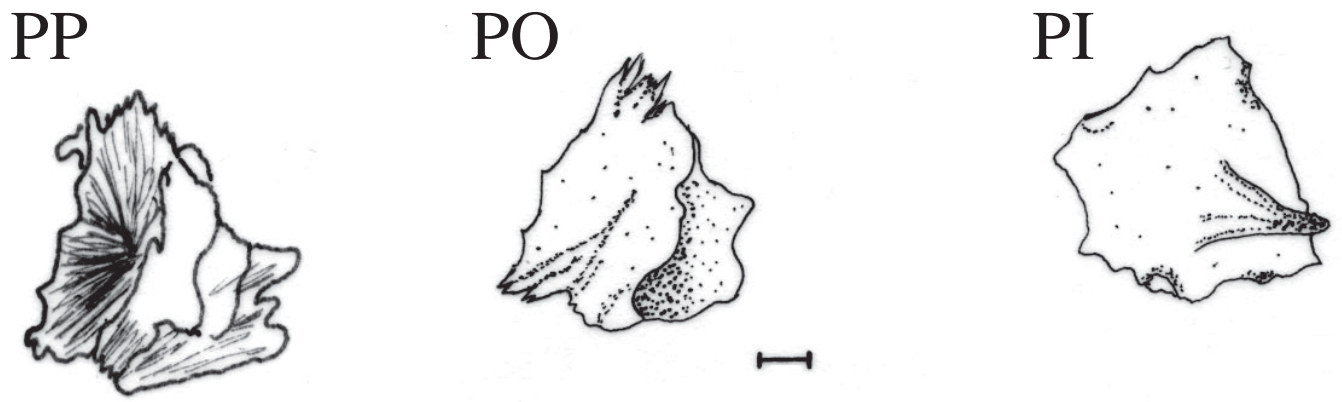

Fig. 16: Intercalares derechos de Paralichthys patagonicus (PP), P. orbignyanus (PO) y $P$. isosceles (PI) en vista lateral. Escala $1 \mathrm{~mm}$.

Right intercalars of Paralichthys patagonicus (PP), P. orbignyanus (PO) and P. isosceles (PI) in lateral view. Scale bar indicates $1 \mathrm{~mm}$. 
desplazamiento es mayor por lo que las partes basales derecha e izquierda son asimétricas entre sí. En $P$. orbignyanus existe hacia la derecha de la cresta supraoccipital otra pequeña quilla mediodorsal, más corta y baja (Fig. 18B). Dicha quilla está ausente en los huesos de $P$. patagonicus y $P$. isosceles.

Los parietales están ubicados dorsalmente en la parte posterior de la caja craneana. Están articulados con los frontales hacia adelante y con los epioccipitales por detrás. Están superpuestos ampliamente al borde de los pteróticos delimitando junto a estos el borde posterolateral del neurocráneo (Fig. 1). Por sus bordes internos están articulados por sinartrosis al supraoccipital. Ambos parietales no son del todo simétricos. El derecho, ligeramente más pequeño, presenta una cresta que se continúa con el epioccipital, más elevada que el correspondiente al parietal izquierdo. La relación longitud/ancho del hueso es de 2,93 en $P$. orbignyanus, 2,75 en $P$. patagonicus y 2,36 en $P$. isosceles (Fig. 19C).

El basioccipital se halla ubicado en la parte posteroventral del neurocráneo. Posee el cóndilo

\section{PP}

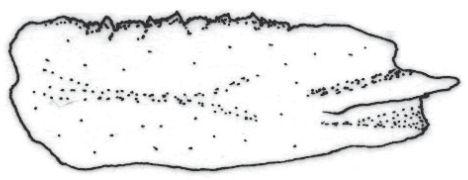

$\mathrm{PO}$

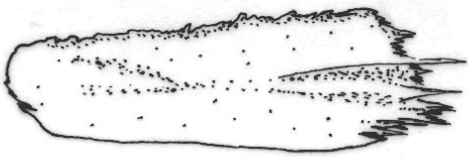

PI

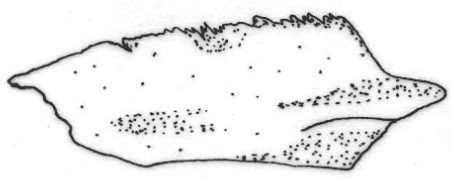

Fig. 17: Exoccipitales izquierdos de Paralichthys patagonicus (PP), P. orbignyanus (PO) y $P$. isosceles (PI) en vista lateral. Escala $1 \mathrm{~mm}$.

Left exoccipitals of Paralichthys patagonicus (PP), $P$ orbignyanus (PO) and $P$. isosceles $(\mathrm{PI})$ in lateral view. Scale bar indicates $1 \mathrm{~mm}$ de articulación con la primera vértebra formando con su cara dorsal el piso del foramen magnum. Dos crestas dorsolaterales están articuladas, cada una de ellas, con los exoccipitales situados inmediatamente por encima. El extremo dorsoanterior de dichas crestas se ponen en contacto ligeramente con una proyección posteroventral del pterótico. Los basioccipitales en las tres especies de Paralichthys son similares entre sí. Las pocas diferencias halladas se refieren al borde posterodorsal del hueso que forma el piso del foramen magnum y a la ubicación de la apertura u orificio de salida del notocordio. En $P$. isosceles dicho borde presenta una concavidad o fosa, ausente en las otras dos especies (Fig. 20A). El orificio para el paso del notocordio está situado centralmente en el cóndilo del basioccipital en $P$. isosceles, mientras que en $P$. patagonicus y $P$. orbignyanus está desplazado hacia arriba (Fig. 20B).

El parasfenoides recorre longitudinalmente la base del neurocráneo desde el basioccipital hasta la región olfatoria. Woolcott et al. (1968) y Pérez \& Werner (1986) señalan dos torsiones en el hueso: una conspicua hacia la derecha, a la altura de la fosa hiomandibular, y la segunda menor cerca del borde posterior de la órbita izquierda. En el presente caso, hay una sola torsión, que se manifiesta en la parte anterior, hacia el lado ciego, antes de encontrar al prevómer (Fig. 1B). El parasfenoides presenta un proceso laminar ascendente, de cada lado, en su parte media que está articulado por sinartrosis con el frontal hacia adelante, el pterosfenoides en la parte media y el proótico por detrás. Estos procesos se unen hacia abajo constituyendo una quilla ventral a cuyos lados se origina un poderoso músculo de la cavidad bucal: el adductor arcus palatini. Anteriormente está articulado fuertemente con el paretmoides derecho, pero ligeramente con el izquierdo. Una larga excavación, que se extiende hacia atrás, recibe la proyección posterior y espinosa del prevómer. Posteriormente su cara dorsal está articulada por sincondrosis con el basioccipital. El proceso laminar del lado izquierdo se encuentra más desarrollado que el correspondiente al lado derecho en $P$. isosceles. En las otras dos especies, dichos procesos son prácticamente similares en tamaño. El proceso lateral izquierdo, en relación a la longitud del hueso es más largo en $P$. patagonicus y $P$. orbignyanus $(28-30 \%)$ que en $P$. isosceles $(20-24 \%)$ (Fig. 21). 

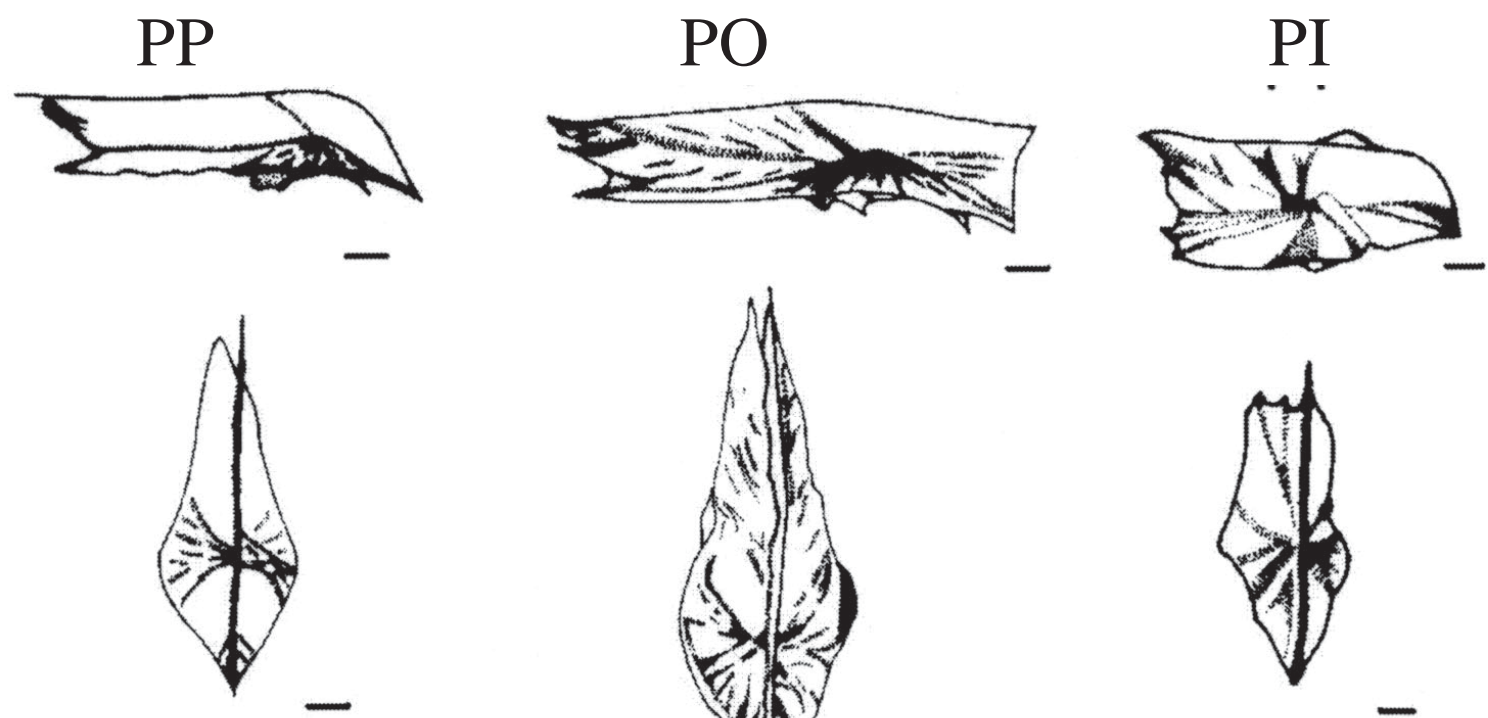

Fig. 18: Supraoccipitales de Paralichthys patagonicus (PP), P. orbignyanus (PO) y P. isosceles (PI) en vistas lateral (arriba) y dorsal (abajo). Escala $1 \mathrm{~mm}$.

Supraoccipitals of Paralichthys patagonicus (PP), P. orbignyanus (PO) and P. isosceles (PI) in lateral (upper) and dorsal (lower) views. Scale bar indicates $1 \mathrm{~mm}$.

PI

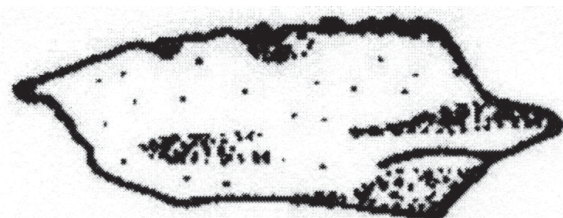

$\mathrm{PO}$

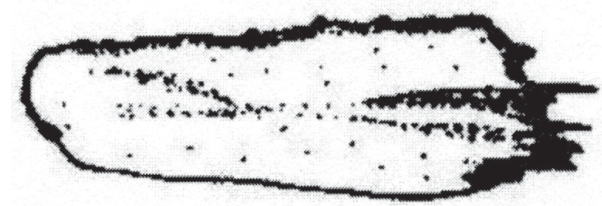

$\mathrm{PP}$

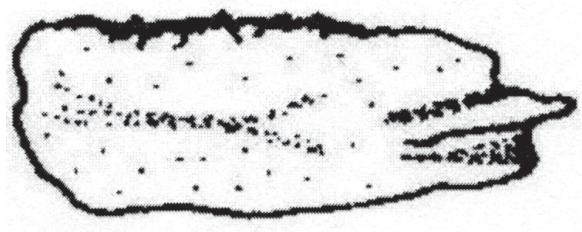

Fig. 19: Parietales izquierdos de Paralichthys patagonicus (PP), P. orbignyanus (PO) y $P$. isosceles (PI) en vista dorsal. Escala $1 \mathrm{~mm}$.

Left parietals of Paralichthys patagonicus (PP), $P$. orbignyanus (PO) and $P$. isosceles (PI) in dorsal view. Scale bar indicates $1 \mathrm{~mm}$.
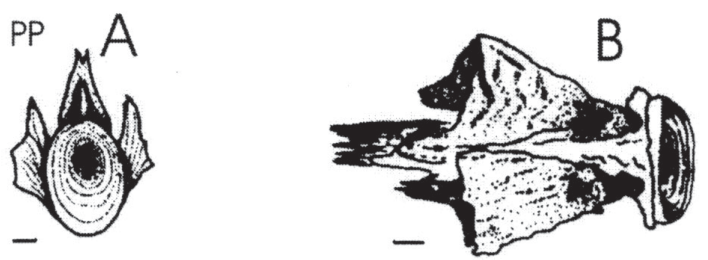

PO
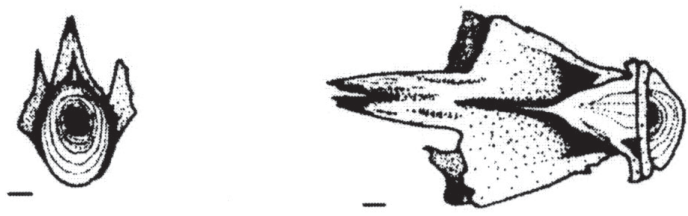

PI
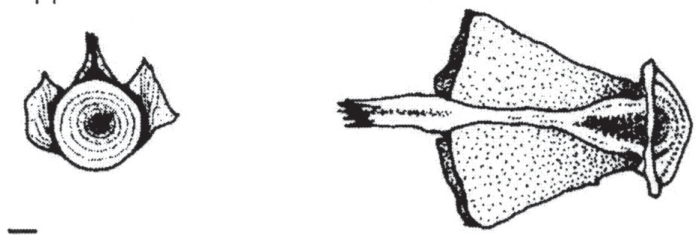

Fig. 20: Basioccipitales de Paralichthys patagonicus (PP), $P$. orbignyanus $(\mathrm{PO})$ y $P$. isosceles (PI) en vistas posterior (A) y dorsal (B). Escala $1 \mathrm{~mm}$.

Basioccipitals of Paralichthys patagonicus (PP), $P$ orbignyanus (PO) and P. isosceles (PI) in rear (A) and dorsal (B) views. Scale bar indicates $1 \mathrm{~mm}$. 


\section{PP}

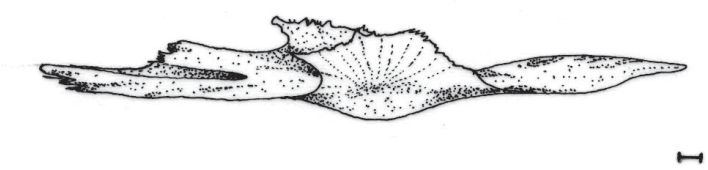

\section{$\mathrm{PO}$}

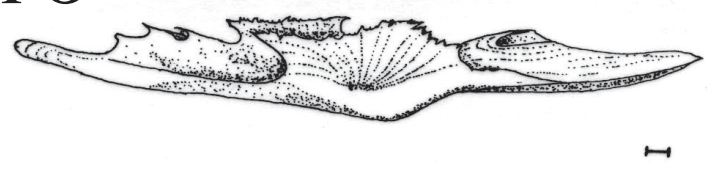

PI

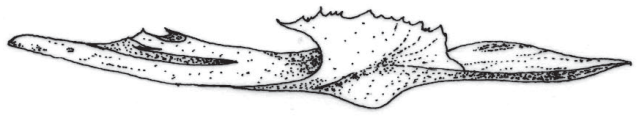

Fig. 21: Parasfenoides de Paralichthys patagonicus $(\mathrm{PP}), P$. orbignyanus $(\mathrm{PO})$ y $P$. isosceles $(\mathrm{PI})$ en vista lateral. Escala $1 \mathrm{~mm}$.

Parasphenoids of Paralichthys patagonicus (PP), $P$ orbignyanus (PO) and $P$. isosceles $(\mathrm{PI})$ in lateral view. Scale bar indicates $1 \mathrm{~mm}$.
Los postemporales unen la cintura escapular al cráneo por medio de los supracleitra. El extremo superior se apoya sobre la superficie dorsal de la proyección lateral del epioccipital. Anteroventralmente se unen a través de un fuerte ligamento a los intercalares. En sentido oblicuo presentan un canal a través del cual pasa el sistema laterosensorial proveniente del cuerpo. La forma del hueso es la de un martillo aplanado con la cabeza dirigida hacia abajo. Posteroventralmente presentan una muesca interna que une fuertemente el postemporal con el supracleitrum.

Los huesos derecho e izquierdo son simétricos en las tres especies estudiadas. Existe un proceso espinoso dirigido anteriormente en el tercio inferior de la cara interna del hueso Dicho proceso está bien desarrollado en $P$. patagonicus y $P$. isosceles, pero menos evidente en $P$. orbignyanus. La anchura del hueso en relación a su longitud, es mayor en $P$. isosceles (55-58\%) que en $P$. orbignyanus y $P$. patagonicus $(47-50$ \%) (Fig. 22).

Los extraescapulares son pequeños huesos pares, de canal, cilíndricos, uno con forma de "Y" (extraescapular lateral) y el otro tubular (extraescapular dorsal) (Fig. 23). Están situados en el extremo posterolateral del neurocráneo en

\section{PP}

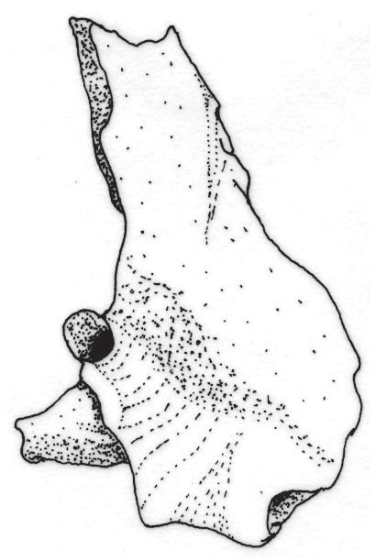

PO

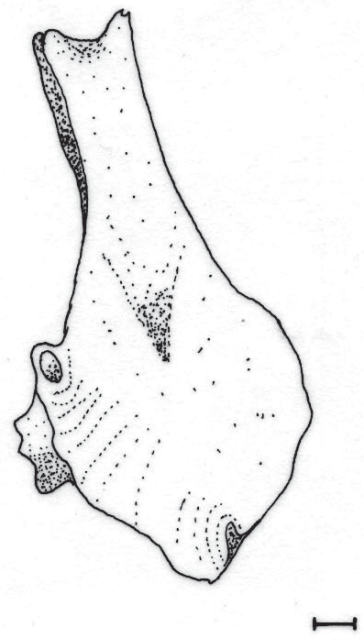

PI

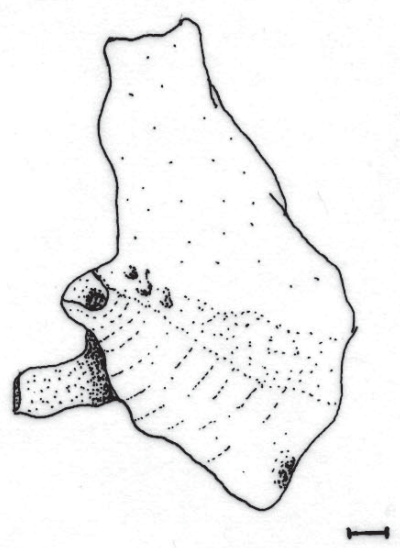

Fig. 22: Postemporales izquierdos de Paralichthys patagonicus (PP), P. orbignyanus (PO) y $P$. isosceles (PI) en vista dorsal. Escala $1 \mathrm{~mm}$.

Left postemporals of Paralichthys patagonicus (PP), P. orbignyanus (PO) and P. isosceles (PI) in dorsal view. Scale bar indicates $1 \mathrm{~mm}$. 


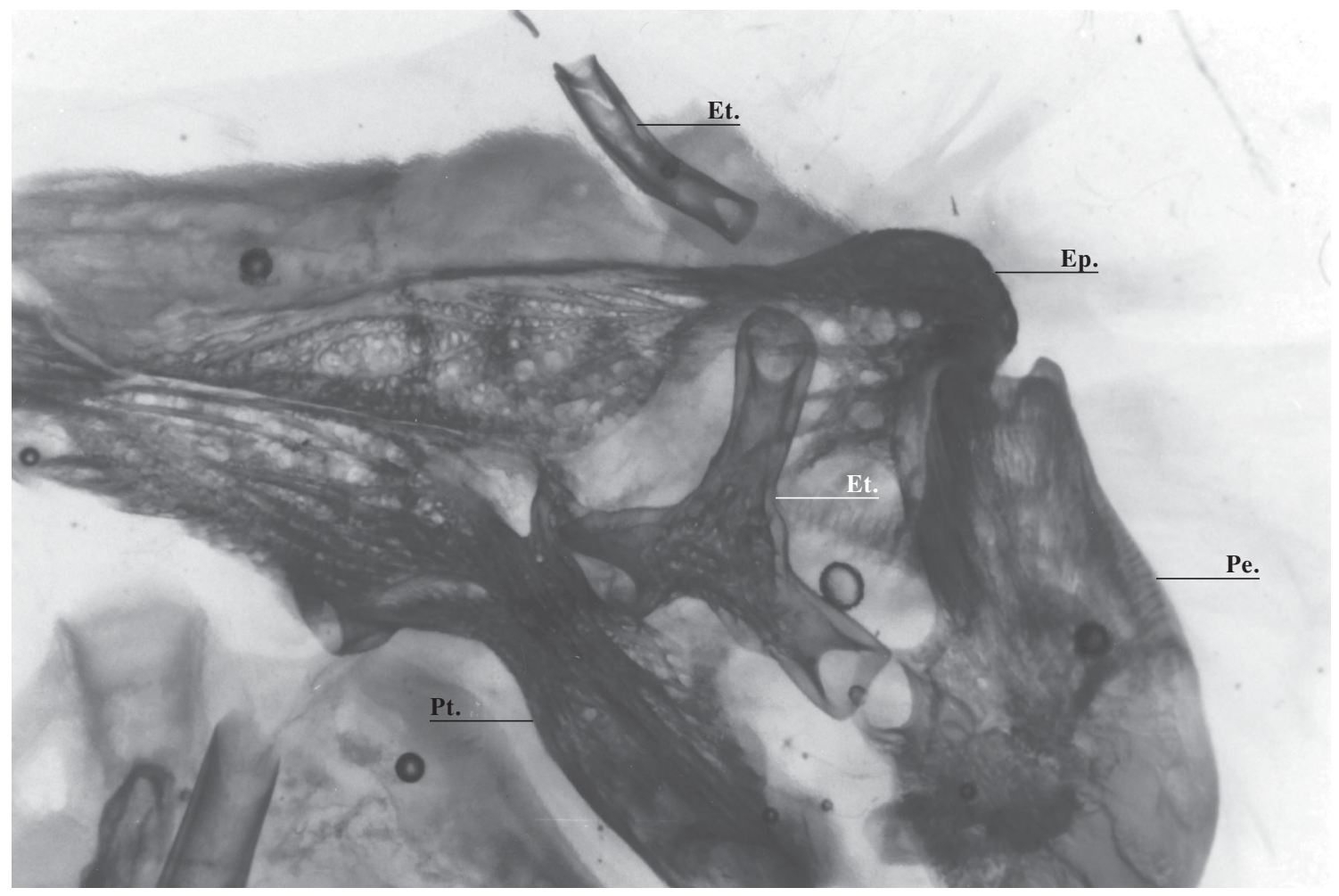

Fig. 23: Parte posterolateral de un neurocráneo de Paralichthys isosceles teñido y diafanizado, mostrando los huesos extraescapulares (Et.). Ver otras abreviaturas en el texto. Escala $1 \mathrm{~mm}$.

Posterolateral part of a cleared and stained neurocranium of Paralichthys isosceles showing the tube-like extrascapulars (Et.). See others abbreviations on the text. Scale bar indicates $1 \mathrm{~mm}$.

una leve concavidad formada por el epioccipital, la porción posterodorsal del pterótico y parte del parietal. Presentan tres orificios que marcan la unión de los canales sensoriales temporal (postorbital), supratemporal y postemporal. La rama temporal (postorbital) del extrascapular lateral es más corta y evidente en $P$. isosceles y $P$. orbignyanus, y poco notable en $P$. patagonicus (Fig. 24B).

\section{Características generales del branquiocráneo}

La asimetría de los elementos del branquiocráneo no es tan marcada como la del neurocráneo, y aunque indirectamente está relacionada con la torsión del cráneo por la migración ocular, parece consecuencia más directa de la morfología funcional y del diferente mecanismo del movimiento de las mandíbulas de cada lado, como lo explica Yazdani (1969) en su trabajo sobre la función adaptativa de las mandíbulas de los
Pleuronectiformes con los hábitos alimenticios. El branquiocráneo (Fig. 25) para un mejor análisis comparativo, fue dividido en cinco regiones: mandibular, palatocuadrado, hioideo, opercular y branquial.

\section{Arco mandibular}

Esta región está compuesta por la mandíbula superior, constituida por los huesos premaxilar y maxilar, y la mandíbula inferior, formada por los huesos dentario, anguloarticular y retroarticular (Fig. 26). El premaxilar y dentario son los únicos elementos de la serie que llevan dientes.

El premaxilar (Fig. 27) constituye el borde superior de la boca. Lleva tres procesos que lo caracterizan: el proceso ascendente, anterior, de posición vertical y comprimido lateralmente. Inmediatamente por detrás está el proceso articular, de posición oblicua, más corto y ancho, que se acopla dentro de una profunda 
PP

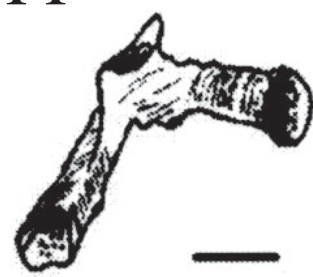

$\mathrm{PO}$

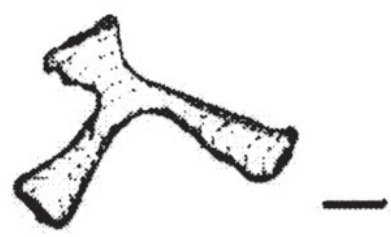

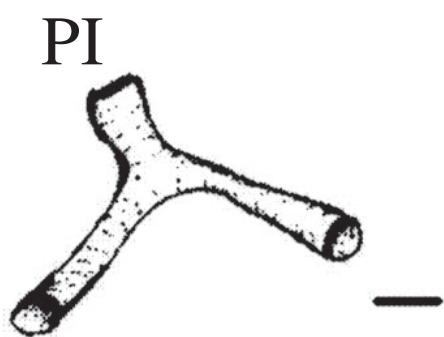

Fig. 24: Extraescapulares laterales izquierdos de Paralichthys patagonicus (PP), P. orbignyanus (PO) y $P$. isosceles (PI). Escala $1 \mathrm{~mm}$.

Left lateral extrascapulars of Paralichthys patagonicus (PP), P. orbignyanus (PO) and P. isosceles (PI). Scale bar indicates $1 \mathrm{~mm}$.

concavidad de la cabeza del maxilar. El tercero es el proceso maxilar del premaxilar, ubicado aproximadamente en la mitad del hueso; es de base ancha y de aspecto laminar. Los huesos derecho e izquierdo presentan unas leves asimetrías en $P$. patagonicus y $P$. orbignyanus. El proceso ascendente del lado oculado es un poco más largo, mientras que el proceso articular es más pequeño en el lado ciego. En $P$. orbignyanus el número de dientes del premaxilar izquierdo es superior al correspondiente del lado derecho (rango 29 - 42 y media 30,7 versus 19 27 y 22 , respectivamente). En P. isosceles no se observaron diferencias entre los huesos de ambos lados. Las diferencias encontradas entre los premaxilares de las tres especies fueron las siguientes (Fig. 27): (1) el proceso ascendente es relativamente más largo en $P$. orbignyanus que en las otras dos especies. (2) Los primeros dos dientes caniniformes son ligeramente más grandes que el resto de los dientes premaxilares en $P$. patagonicus y $P$. orbignyanus; en $P$. isosceles los primeros dientes no son mucho más grandes que el resto, y se van reduciendo de tamaño gradualmente. (3) El proceso ascendente del premaxilar se halla muy cerca del extremo anterior del hueso en $P$. isosceles, mientras que en $P$. patagonicus y $P$. orbignyanus, dicho proceso está un poco por debajo del extremo anterior. (4) Paralichthys patagonicus presentó un mayor número de dientes (rango 25 - 46, media 35,6, desvío estándar 4,7 y n $=35)$ que $P$. isosceles $(26$ - 49;31,2;3,5 y 23) y $P$. orbignyanus ( 25 - 42; 30,7; 5,5 y 10). Woolcott et al. (1968) no encuentran diferencias significativas en el número de dientes premaxilares en las tres especies que los autores estudiaron ( $P$. dentatus, $P$. lethostigma y $P$. albigutta).
El maxilar presenta dos procesos, dorsal o craneal y ventral o premaxilar, bien desarrollados. El proceso ventral delimita una profunda fosa de articulación con el premaxilar (Fig. 26). La parte media del hueso es aproximadamente cilíndrica y se continúa con una expansión laminar en el extremo posterior. En el borde superior del brazo del maxilar existe un pequeño proceso dirigido hacia atrás donde se inserta el ligamento primordial que se une con el tendón del músculo aductor mandibular. En las tres especies estudiadas el maxilar del lado ciego resultó ser más robusto, sobre todo en la parte media del hueso. El proceso espinoso en el cual está inserto el ligamento primordial está más desarrollado en el hueso de ese mismo lado. Las diferencias entre los maxilares de las tres especies son (Fig. 28): (1) El borde laminar del extremo posterior del hueso es oblicuo en relación a su eje longitudinal en $P$. isosceles, siendo recto y perpendicular a dicho eje en $P$. patagonicus y $P$. orbignyanus. (2) El proceso espinoso premaxilar del maxilar constituye una concavidad en forma de $\mathrm{V}$, bien notoria en $P$. isosceles, menos evidente en $P$. patagonicus y ausente en $P$. orbignyanus. (3) Una profunda fosa en el ángulo inferior entre el proceso premaxilar y el eje longitudinal del hueso está presente en $P$. isosceles, pero ausente en las otras dos especies. (4) La cabeza del maxilar, medida hasta el punto donde se inserta el ligamento primordial, en relación al largo total del hueso, es más larga en $P$. patagonicus (33 $35 \%$ ) que en $P$. isosceles (30 - $32 \%$ ), pero más corta que en $P$. orbignyanus (34 - $37 \%$ ). En otras especies de Paralichthys del Atlántico Noroccidental, los valores obtenidos fueron: 33 $\%$ para $P$. dentatus y $40 \%$ para $P$. lethostigma y $P$. albigutta (Woolcott et al. 1968). 

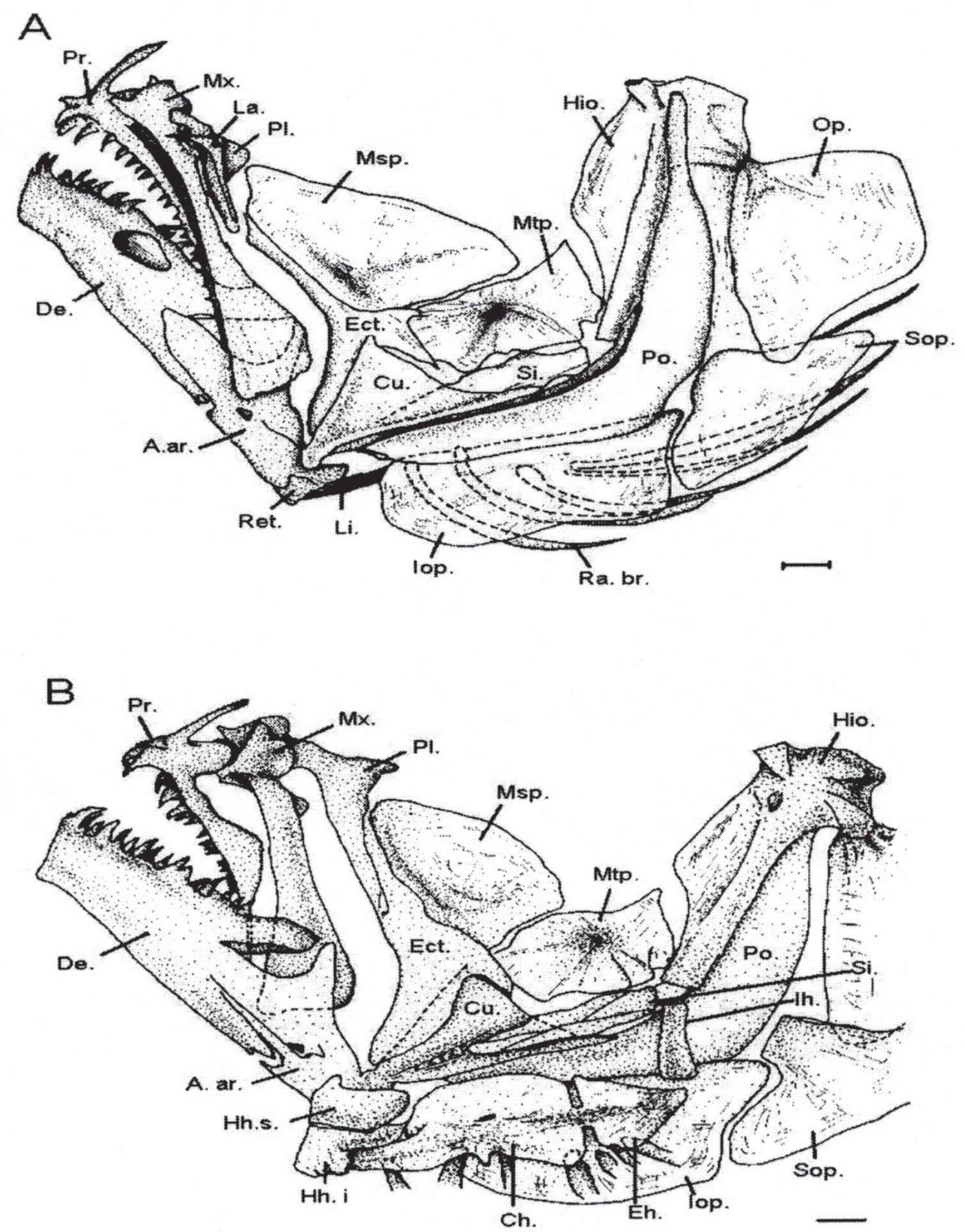

Fig. 25: Branquiocráneo de Paralichthys isosceles. A: lado izquierdo, vista externa; B: lado derecho, vista interna. Ver abreviaturas en el texto.

Branchiocranium of Paralichthys isosceles. A: left side, outer view. B: right side, inner view. See abbreviations on text. Scale bar indicates $1 \mathrm{~mm}$. 


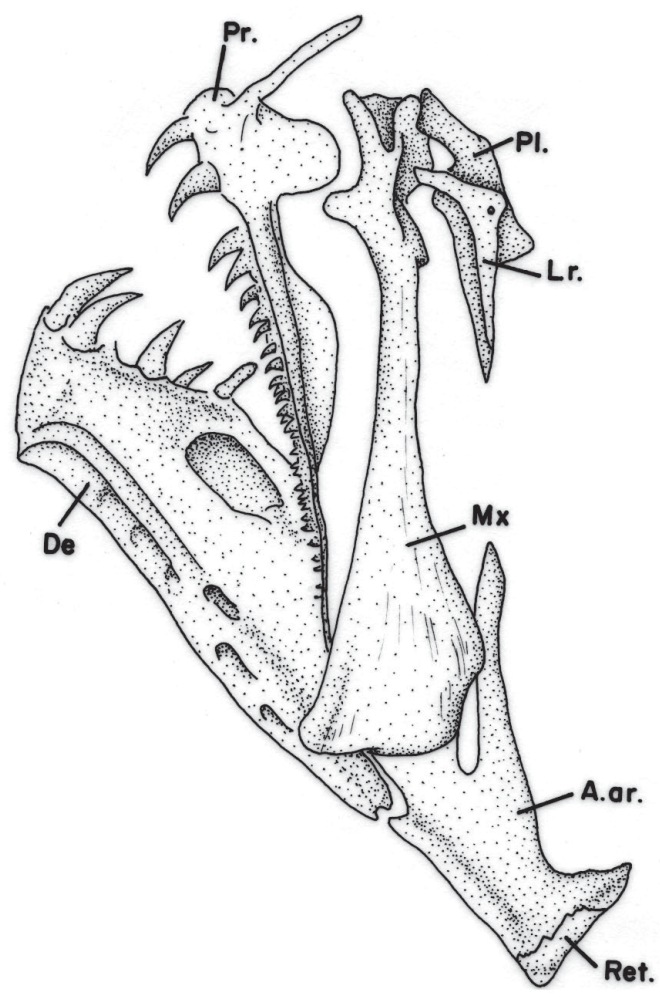

Fig. 26: Arco mandibular de Paralichthys patagonicus. Ver abreviaturas en el texto. Escala $1 \mathrm{~mm}$.

Mandibular arch of Paralichthys patagonicus. See abbreviations on text. Scale bar indicates $1 \mathrm{~mm}$.

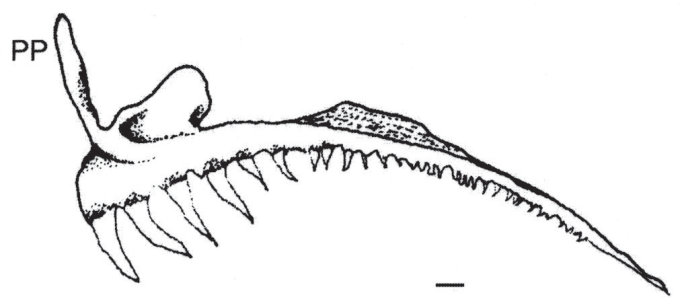

$\mathrm{PO}$

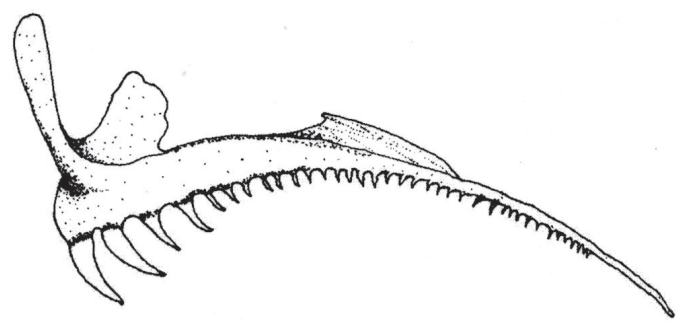

PI

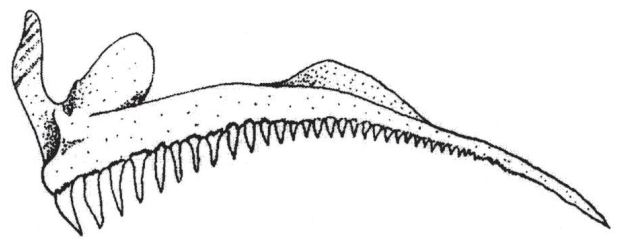

Fig. 27: Premaxilares de Paralichthys patagonicus (PP), $P$. orbignyanus (PO) y $P$. isosceles (PI) en vista lateral del lado oculado. Escala $1 \mathrm{~mm}$.

Premaxillas of Paralichthys patagonicus (PP), $P$. orbignyanus (PO) and $P$. isosceles (PI) in lateral view from the eyed side. Scale bar indicates $1 \mathrm{~mm}$.

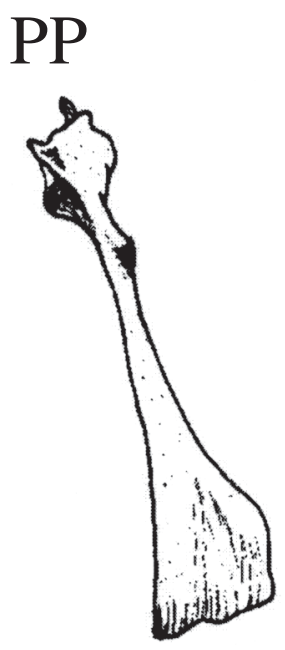

PO

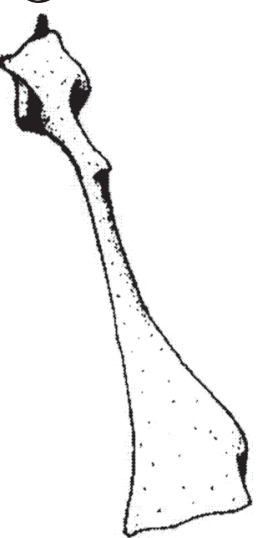

PI

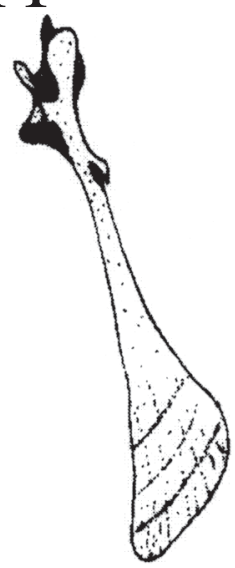

Fig. 28: Maxilares izquierdos de Paralichthys patagonicus (PP), P. orbignyanus (PO) y P. isosceles (PI) en vista lateral. Escala $1 \mathrm{~mm}$.

Left maxillas of Paralichthys patagonicus (PP), P. orbignyanus (PO) and P. isosceles (PI) in lateral view. Scale bar indicates $1 \mathrm{~mm}$. 
El dentario forma el borde inferior de la boca. Lleva dientes cónicos muy puntiagudos. Es de forma aproximadamente triangular con su borde posterior hendido en forma de horqueta constituyendo dos ramas, superior e inferior. Presenta un gran poro oval ubicado en la superficie laterodorsal cerca del centro del hueso. En el borde posterodorsal sobresale una proyección que se relaciona con el proceso coronoides del anguloarticular. Sobre la cara interna se visualiza una profunda concavidad donde se aloja el cartílago de Meckel y el extremo anterior del angular (Fig. 29). La longitud del dentario del lado oculado es más corta que la correspondiente al hueso del lado ciego en un $8 \%$ para $P$. orbignyanus, 8,3\% para $P$. patagonicus y 8,5\% para $P$. isosceles. La relación diámetro horizontal del poro oval y la longitud del dentario dio los siguientes valores: $10,8 \%$ en $P$. orbignyanus, 17 $\%$ en $P$. patagonicus y $20 \%$ en $P$. isosceles. El tamaño del poro fue casi el doble en $P$. isosceles que en $P$. orbignyanus (Fig. 30, izquierda). Si bien el número de dientes fue constante entre los huesos derecho e izquierdo en las tres especies, hubo diferencias merísticas interespecíficas. Paralichthys isosceles presentó los valores más altos $($ rango $=16-21 ;$ media $=18,5 ; \mathrm{DE}=2,6 ; \mathrm{n}=$ 10 ), le siguen $P$. orbignyanus (rango $=9-13$; media $=10,9 ; \mathrm{DE}=1,2 ; \mathrm{n}=23$ ) y P. patagonicus (rango $=6-9 ;$ media $=7,4 ; \mathrm{DE}=0,9 ; \mathrm{n}=35)$. Woolcott et al. (1968) encontraron resultados semejantes para la separación de las especies de Paralichthys por ellos estudiadas.

El extremo anterior, sumamente agudo, del anguloarticular se inserta en la concavidad del dentario. Presenta tres grandes procesos: dorsal o coronoides, dirigido anterodorsalmente; el posterior, también orientado hacia arriba y con una fosa de articulación para el cuadrado; y el proceso vertebral dirigido hacia adelante. Ventrolateralmente una serie de poros abren paso al canal sensorial proveniente del preopérculo y que se continúa en el dentario. En su parte interna y fuertemente adherido a la superficie cóncava, el anguloarticular lleva un pequeño huesecillo, más o menos alargado, llamado coronomeckeliano o sesamoide articular (Fig. 29). Sobre él se inserta el tendón de la división $\mathrm{A}_{2}$ del aductor mandibular, responsable en parte del mecanismo de cierre de la boca. Muchas veces este elemento pasa inadvertido y de ahí que en muchos trabajos no sea descripto. El cartílago de Meckel está reducido a una varilla cartilaginosa, que se inserta en una concavidad de la cara interna del anguloarticular y se prolonga hasta cerca de la cara interna del dentario (Fig. 29). En las tres especies el hueso del lado ciego resultó ser ligeramente más largo que el correspondiente al lado oculado. Las variaciones morfológicas interespecíficas fueron las siguientes (Fig. 30, derecha): (1) El proceso coronoides presenta una inclinación hacia adelante más pronunciada en $P$. isosceles que en las otras dos especies. (2) El borde anterior del proceso coronoides muestra una ondulación en $P$. orbignyanus y ausente en $P$. patagonicus y $P$. isosceles. (3) La relación longitud del canal óseo que encierra la rama sensorial anguloarticular, y la longitud total del hueso fue mayor en $P$. orbignyanus $(38 \%)$ y menor en $P$. patagonicus $(30 \%)$ y $P$. isosceles $(21 \%)$. (4) La altura del anguloarticular, medida desde el extremo del proceso coronoides al extremo del ventral, en relación al largo total del hueso fue de $47 \%$ en $P$. patagonicus y $P$. orbignyanus y $43 \%$ en $P$. isosceles.

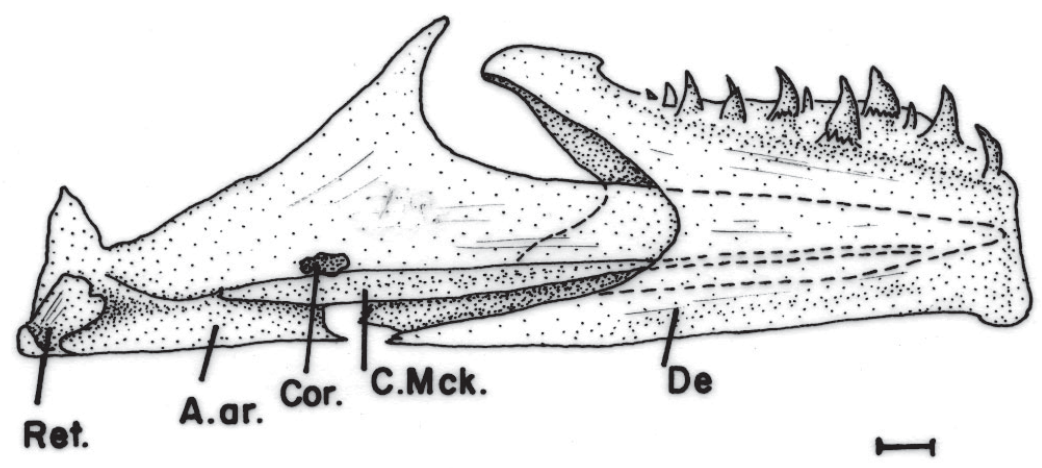

Fig. 29: Vista interna de la mandíbula inferior derecha de Paralichthys patagonicus. Ver abreviaturas en el texto. Escala $1 \mathrm{~mm}$.

Inner view of the right lower jaw of Paralichthys patagonicus. See abbreviations on text. Scale bar indicates $1 \mathrm{~mm}$. 


\section{PP}
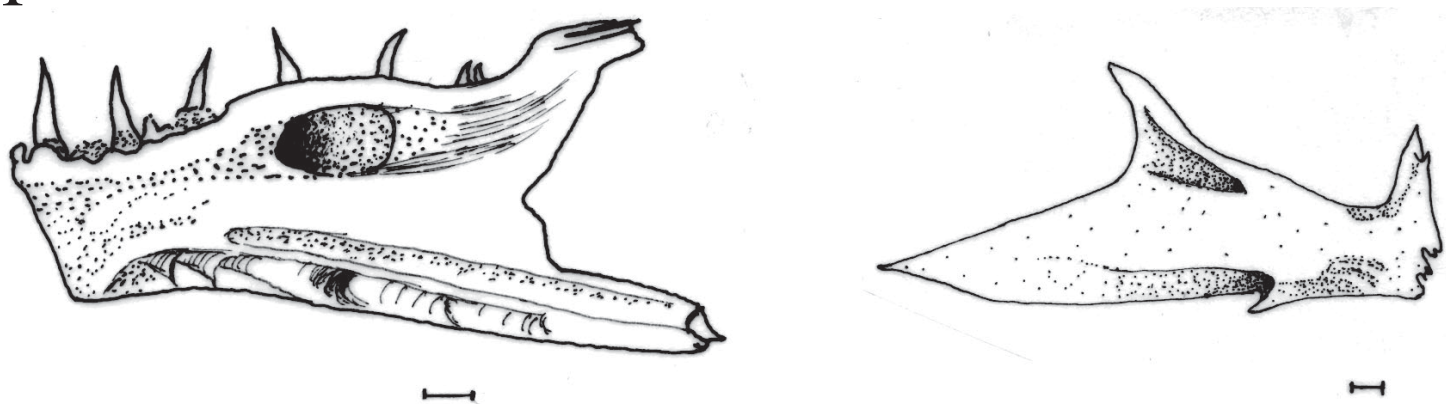

\section{$\mathrm{PO}$}
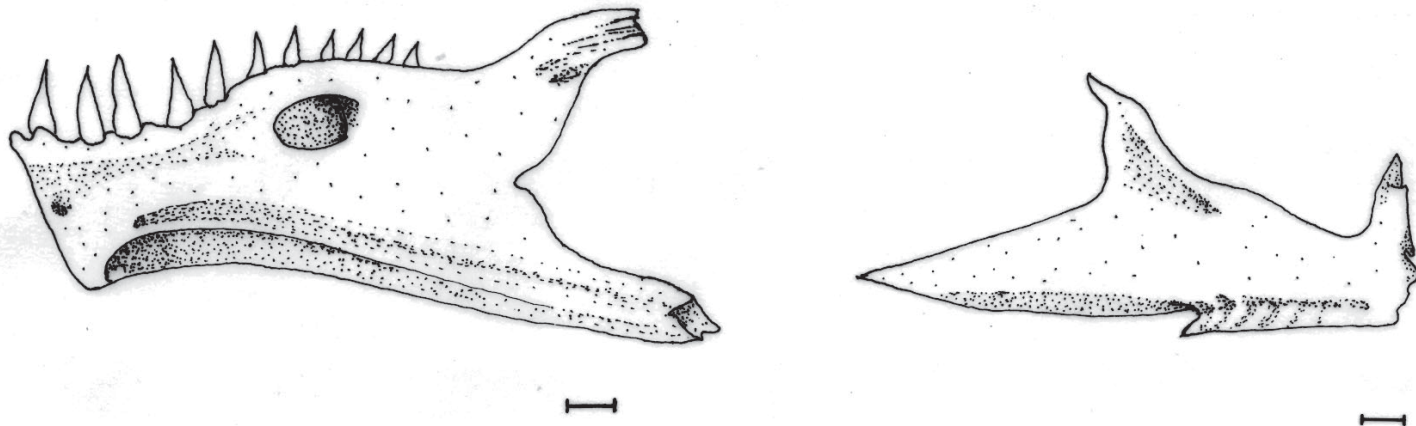

PI
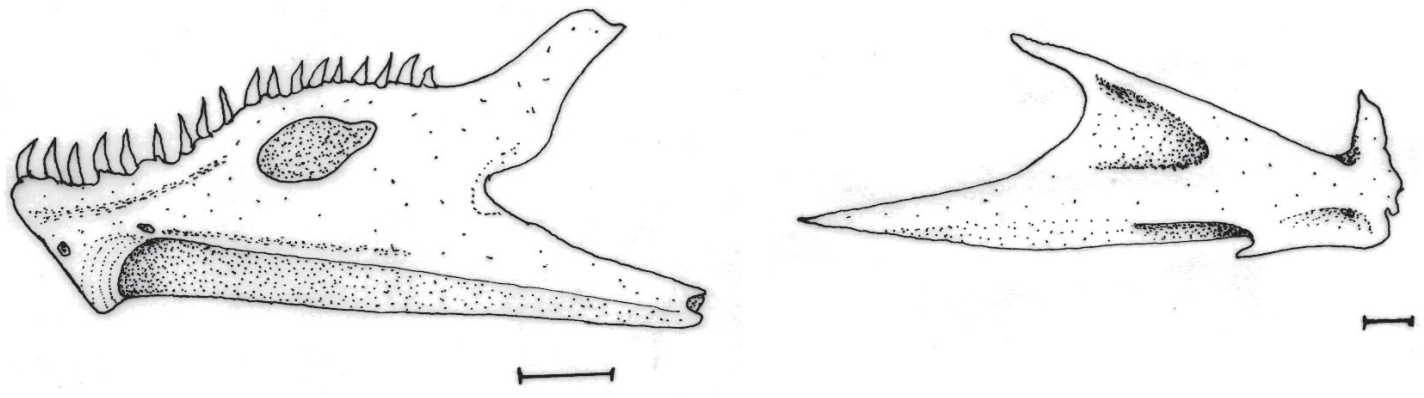

Fig. 30: Dentarios (izquierda) y anguloarticulares (derecha) de Paralichthys patagonicus (PP), $P$. orbignyanus $(\mathrm{PO})$ y $P$. isosceles $(\mathrm{PI})$ en vista lateral. Escala $1 \mathrm{~mm}$.

Dentary bones (left) and anguloarticular bones (right) of Paralichthys patagonicus (PP), P. orbignyanus (PO) and P. isosceles (PI) in lateral view. Scale bar indicates $1 \mathrm{~mm}$. 
El retroarticular está situado en el ángulo posteroinferior del anguloarticular y recibe el ligamento proveniente del interopérculo (Fig. 31). Presenta dos procesos espinosos, uno superior más desarrollado y otro inferior. No se hallaron diferencias entre los huesos de los dos lados en ninguna de las tres especies estudiadas.

\section{Arco palatocuadrado}

El arco está formado por los huesos pares palatino, ectopterigoides, mesopterigoides, metapterigoides, cuadrado, simpléctico e hiomandibular. Está articulado al neurocráneo anteriormente por el contacto palatinoprevómer y posteriormente, a través del arco hioideo, por la articulación por diartrosis metapterigoides-hiomandibular.

Los palatinos son huesos edéntulos, asimétricos; el derecho es más grande que el izquierdo (Fig. 32). Tienen la forma de un "martillo con mango corto" (palatino izquierdo) y con "mango largo" (palatino derecho). Por el extremo anterior se relacionan dorsalmente con el paretmoides y mesetmoides a través de un ligamento, y ventralmente, en una ligera depresión, se inserta el fuerte ligamento que une el palatino al borde del maxilar. Por el lado interno también se unen por un ligamento ancho y fuerte con el prevómer. Posteroventralmente están articulados por sincondrosis con el ectopterigoides y dorsalmente tocan ligeramente la proyección anterior del mesopterigoides. El lacrimal cubre parcialmente al palatino (Fig. 26). En el lado oculado, el ancho de la cabeza del palatino, en relación a la longitud del hueso es más larga en $P$. orbignyanus $(22 \%)$ que en $P$.patagonicus $(18 \%)$ y $P$. isosceles (12\%) (Fig. 32, arriba). En el lado ciego, el "mango" del palatino es más largo en $P$. isosceles (60-61\% de la longitud del hueso) que en $P$. orbignyanus (50-52\%) y P. patagonicus (48-51 $\%)$ (Fig. 32, abajo).

El ectopterigoides es un hueso curvado en forma de boomerang con un proceso laminar ubicado en la cara convexa de la superficie posterior. Por su extremo anterior está articulado con el "mango" del palatino y con el mesopterigoides por la cara lateral interna. Su rama inferior se prolonga por toda la longitud del borde anterior del cuadrado, al cual está íntimamente unido por sincondrosis y el proceso laminar está articulado por sinartrosis escamosa al metapterigoides (Fig. 25). Woolcott et al. (1968) relacionan a este hueso con el maxilar; sin embargo, en el presente trabajo no se halló vinculación alguna con dicho hueso. Los dos ectopterigoides son asimétricos entre sí. El del lado ciego tiene forma de $\mathrm{L}$ y es más robusto que el del lado oculado (Fig. 33, arriba). La longitud de la rama superior (medida desde el extremo superior al centro del margen anterior) dividida por la longitud de la rama inferior (medida desde el extremo inferior al centro del margen anterior) es 1,2 veces en $P$. isosceles, 1,1 veces en $P$. patagonicus y 1,07 en $P$. orbignyanus. El proceso laminar, ubicado en la cara convexa de la superficie posterior, presenta el borde dorsal liso, curvado hacia abajo y termina en un proceso espinoso en $P$. patagonicus y $P$. orbignyanus. En $P$. isosceles, en cambio, dicho borde está cortado por una escotadura con márgenes aserrados que delimita dos procesos espinosos, uno dorsal y otro ventral (Fig. 33, abajo).

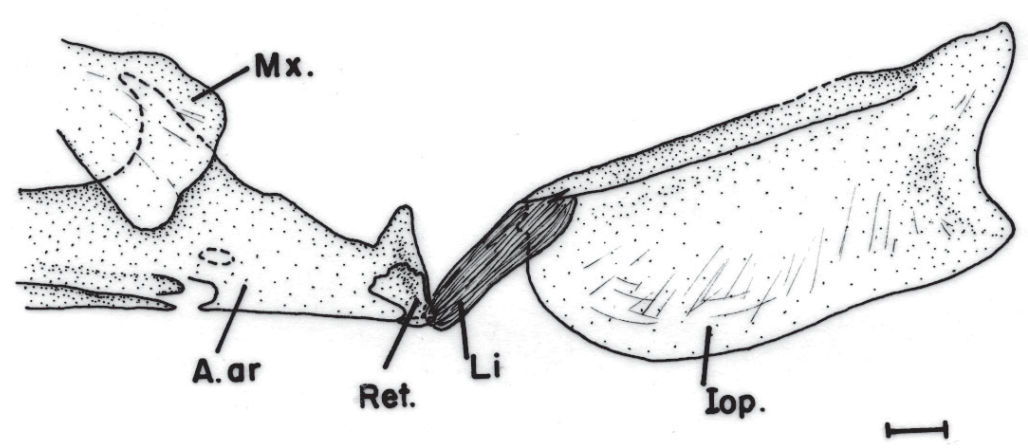

Fig. 31: Vista lateral externa de la conexión mandíbula inferior - serie opercular de Paralichthys patagonicus. Ver abreviaturas en el texto. Escala $1 \mathrm{~mm}$.

Outer lateral view of the lower jaw - opercle series joint in Paralichthys patagonicus. See abbreviations on text. Scale bar indicates $1 \mathrm{~mm}$. 
PP
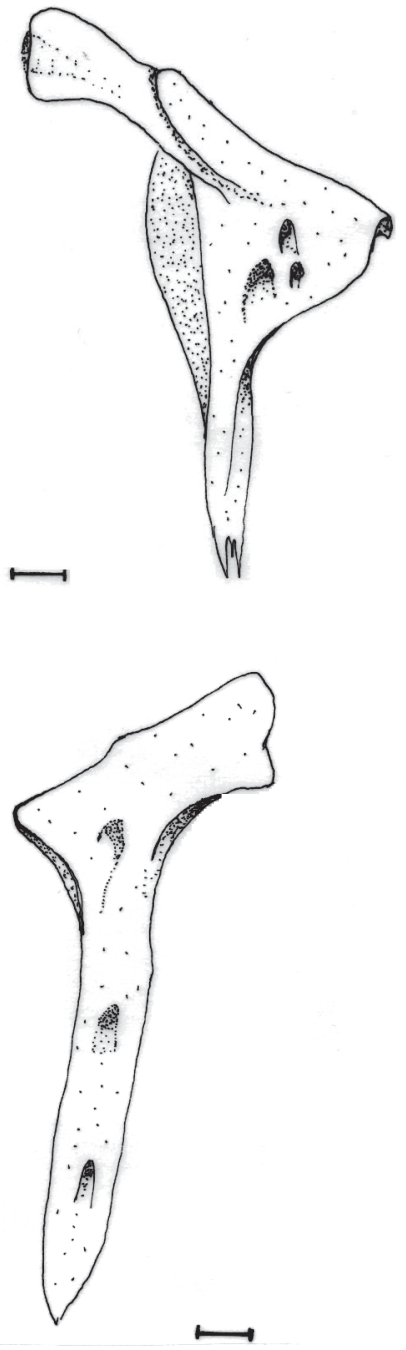

$\mathrm{PO}$
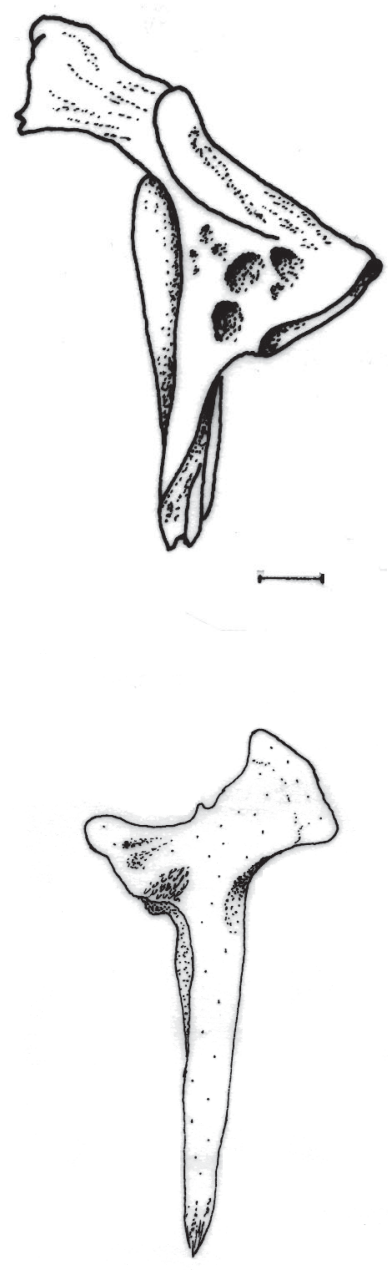

PI

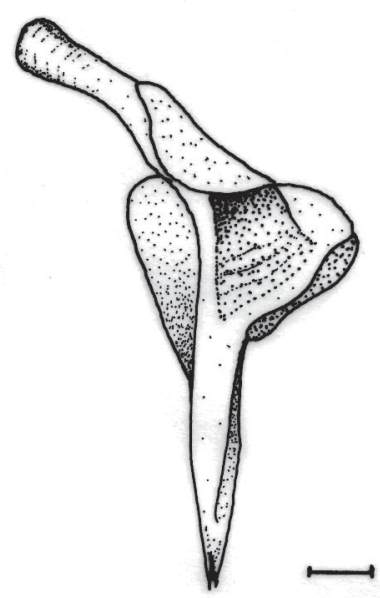

Fig. 32: Huesos palatinos de Paralichthys patagonicus (PP), P. orbignyanus (PO) y P. isosceles (PI) en lados oculado (arriba) y ciego (abajo). Escala $1 \mathrm{~mm}$.

Palatine bones of Paralichthys patagonicus (PP), P. orbignyanus (PO) and P. isosceles (PI) on eyed (upper) and blind (lower) sides. Scale bar indicates $1 \mathrm{~mm}$.

El mesopterigoides, también denominado endopterigoides, es un hueso laminar, de forma oval, en estrecho contacto con la superficie cóncava de la rama superior del ectopterigoides. Hacia atrás se contacta por sinartrosis escamosa con el metapterigoides. Su forma aplanada ayuda a sostener el ojo inferior que no queda contenido en una órbita ósea. No se evidenció contacto alguno entre este hueso y el cuadrado, siendo dicha relación más notoria en otros Pleuronectiformes como en especies de los géneros Achirus y Trinectes (Futch et al. 1972,
Cervigón 1985). Los mesopterigoides derecho e izquierdo son asimétricos entre sí. El derecho es entre un 30 y un $40 \%$ más ancho, en relación al largo del hueso, que el izquierdo en las tres especies de Paralichthys. El mesopterigoides del lado oculado presenta su borde posterior aserrado, con una proyección espinosa, también aserrada, en la cara ventral de $P$. patagonicus. El borde posterior es liso en $P$. orbignyanus como en $P$. isosceles, pero $P$. orbignyanus presenta un proceso espinoso ventral, indistinto o ausente en P. isosceles (Fig. 34). 


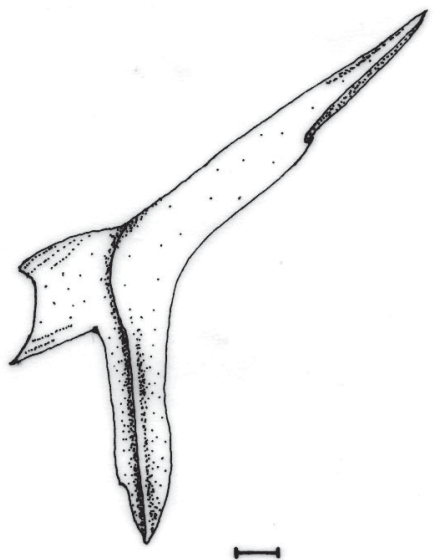

PP

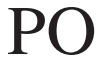

PI
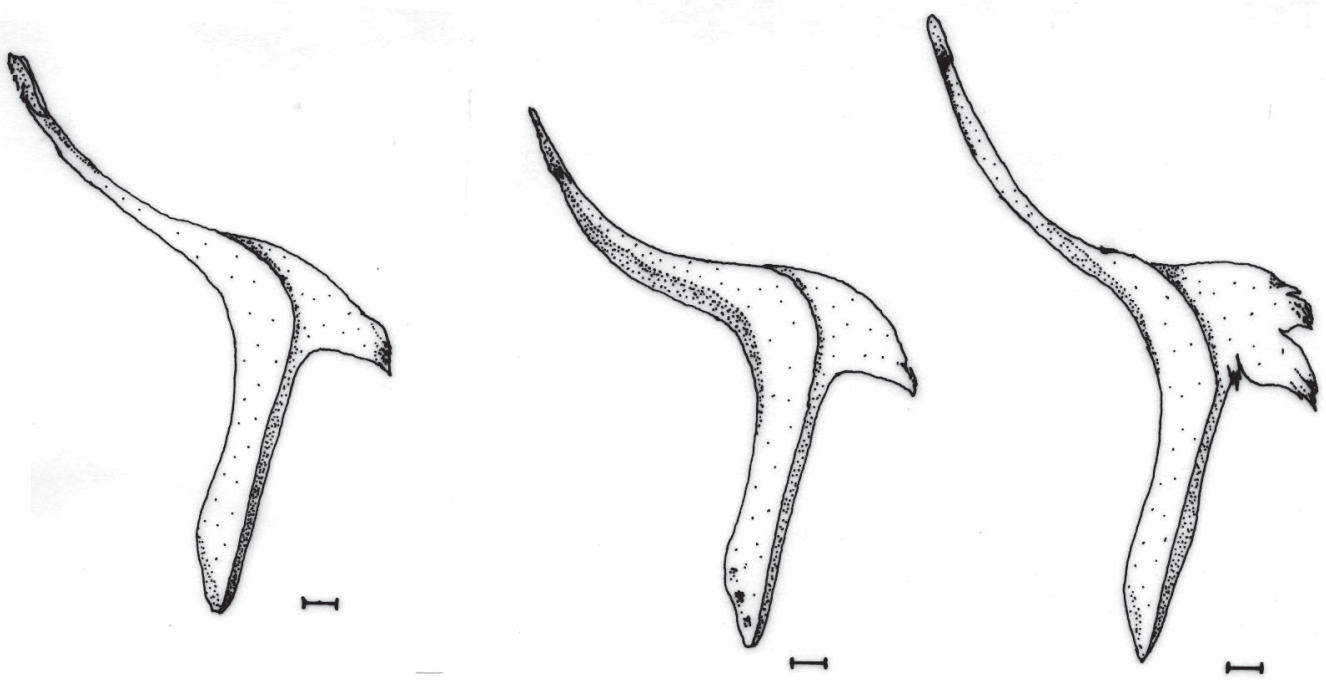

Fig. 33: Ectopterigoides derecho de Paralichthys patagonicus (arriba) y ectopterigoides izquierdos (abajo) de Paralichthys patagonicus (PP), P. orbignyanus (PO) y P. isosceles (PI) en vista lateral. Escala $1 \mathrm{~mm}$.

Right ectopterygoid of Paralichthys patagonicus (upper) and left ectopterygoids (lower) of Paralichthys patagonicus (PP), $P$. orbignyanus $(\mathrm{PO})$ and $P$. isosceles (PI). Scale bar indicates $1 \mathrm{~mm}$.
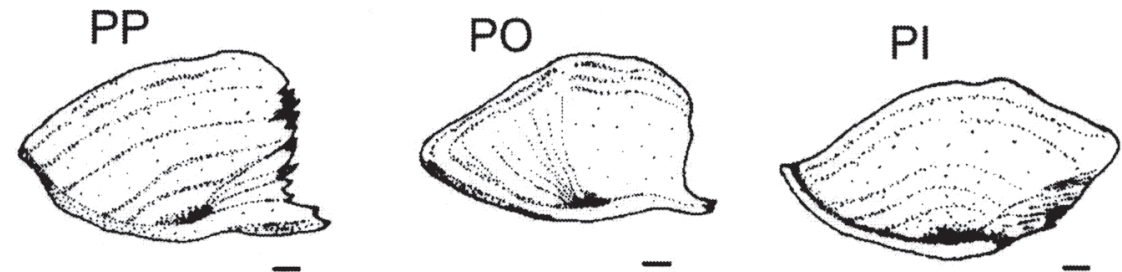

Fig. 34: Mesopterigoides izquierdos de Paralichthys patagonicus (PP), P. orbignyanus (PO) y $P$. isosceles (PI) en vista lateral. Escala $1 \mathrm{~mm}$.

Left mesopterygoids of Paralichthys patagonicus (PP), $P$. orbignyanus (PO) and $P$. isosceles (PI) in lateral view. Scale bar indicates $1 \mathrm{~mm}$. 
El metapterigoides es un hueso aplanado, cuadrangular con bordes irregulares. Anteriormente está articulado por sinartrosis escamosa con el endopterigoides y con el proceso laminar del ectopterigoides. Ventralmente está relacionado con el cuadrado y posteriormente con la parte superior del simpléctico y el extremo inferior del hiomandibular (Fig. 25). La parte superior del borde anterior se proyecta en un proceso espinoso, más desarrollado en $P$. patagonicus y $P$. isosceles, que en $P$. orbignyanus. El margen posterior, que está articulado con el simpléctico, presenta en la parte inferior una escotadura bien notoria en $P$. patagonicus y $P$. orbignyanus y ausente en $P$. isosceles. En esta última especie dicho margen presenta serraciones, ausentes en las otras dos especies del género.

El cuadrado presenta forma triangular. Está articulado anteriormente con el ectopterigoides, posteriormente con el preopérculo y dorsalmente con el metapterigoides y mesopterigoides. En el vértice inferior un amplio cóndilo transversal articula en su correspondiente fosa en el anguloarticular. La cara interna del cuadrado posse un surco profundo que aloja los dos tercios inferiores del simpléctico. La longitud del borde posterior del hueso, incluyendo el proceso espinoso dorsal, en relación al ancho máximo del hueso es de 1,6 veces en $P$. patagonicus, 1,7 en $P$. orbignyanus y 1,5 en $P$. isosceles (Fig. 35, abajo).
PP
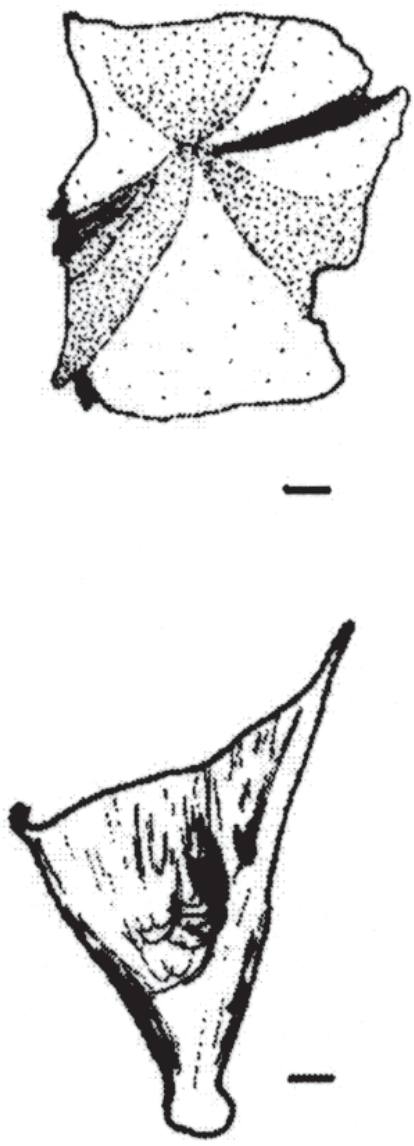

$\mathrm{PO}$
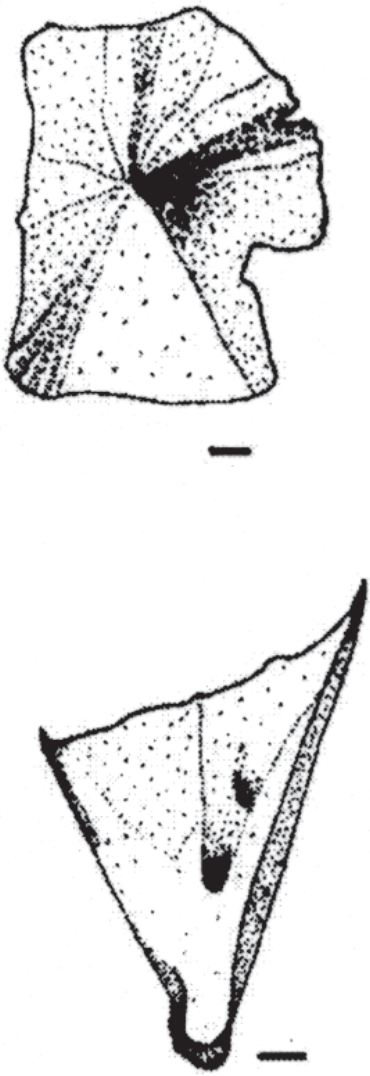

PI

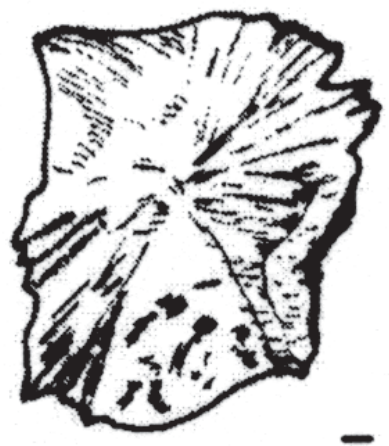

Fig. 35: Metapterigoides (arriba) y cuadrado (abajo) de Paralichthys patagonicus (PP), $P$. orbignyanus (PO) y $P$. isosceles $(\mathrm{PI})$ en vista lateral. Escala $1 \mathrm{~mm}$.

Metapterygoid (upper) and quadrate (lower) bones of Paralichthys patagonicus (PP), P. orbignyanus (PO) and P. isosceles (PI) in lateral view. Scale bar indicates $1 \mathrm{~mm}$. 
El simpléctico es un hueso pequeño, comprimido lateralmente, cuyo extremo inferior, delgado, está insertado a modo de cuña en la cara interna del cuadrado. En sus límites anterior y posterior se encuentran el metapterigoides y preopérculo, respectivamente. Su extremo superior está conectado con el hiomandibular a través de un disco de cartílago (Fig. 25B). El simpléctico tiene forma de bastón con sus bordes anteriores y posteriores más o menos irregulares en $P$. orbignyanus. En $P$. patagonicus presenta en el borde anterior una ligera concavidad y una proyección más o menos espinosa dirigida hacia adelante. En $P$. isosceles no se manifiesta tal concavidad, en cambio, hay una expansión laminar bastante amplia con bordes aserrados (Fig. 38, abajo). La relación ancho/largo del hueso es de 20 - $31 \%$ en $P$. orbignyanus, 22 - $28 \%$ en $P$. patagonicus y $35-50 \%$ en $P$. isosceles.

El hiomandibular es un hueso en forma de L invertida que conecta el arco palatocuadrado y la serie opercular al neurocráneo. Tres procesos de articulación o cóndilos caracterizan el extremo superior del hiomandibular: el primero, de posición dorsoanterior, es un cóndilo cubierto de un disco de cartílago que encaja en una fosa conformada por la unión del esfenótico y proótico; el segundo, ubicado dorsalmente, se inserta en una muesca longitudinal del pterótico, y el tercero, situado posteroventralmente, conecta el hiomandibular con el opérculo. En el extremo inferior del eje longitudinal o "columna" del hiomandibular, una conexión cartilaginosa relaciona al hueso por diartrosis con el simpléctico e interhial (Fig. 25B). Anterolateralmente una expansión laminar une el hiomandibular con el metapterigoides. Posterolateralmente, a lo largo del eje vertical, presenta una superficie de articulación por sincondrosis con el preopérculo. Un fuerte canal o cresta dorsolateral se extiende desde el extremo inferior hasta el superior, donde se curva anteriormente hasta confluir con el cóndilo dorsoanterior. Antes de converger con dicho proceso, el canal se interrumpe delimitando una pequeña espina. El área situada por detrás del canal longitudinal sirve de articulación para el hueso preopercular. En las tres especies de Paralichthys analizadas, el hueso del lado ciego es de un 5 a un $6 \%$ más corto que el del lado oculado. El ancho máximo del hiomandibular (medido desde el extremo del cóndilo anterodorsal al borde exterior del cóndilo ventroposterior), en relación al largo total del hueso (medido desde el extremo ventral al borde dorsal del cóndilo anterodorsal) es 20 . $24 \%$ en $P$. isosceles, $26-28 \%$ en $P$. patagonicus y $30-34 \%$ en $P$. orbignyanus (Fig. 39, arriba). La expansión membranosa anterior del hiomandibular involucra al cóndilo articular dorsoanterior en P. isosceles. En las otras dos especies dicha membrana deja libre la mayor parte del cóndilo. Además, en $P$. isosceles, existe un orificio ubicado en la base del cóndilo anterior, ausente en $P$. patagonicus y $P$. orbignyanus.

\section{Arco hioideo}

Los huesos de esta región conectan los arcos branquiales con el suspensorio, dan soporte esquelético a la lengua y toma parte en los movimientos de apertura de la boca y succión para la alimentación y respiración. El arco hioideo está compuesto de los hipohiales (superior e inferior), ceratohial (ceratohial anterior), epihial (ceratohial posterior), interhial, siete radios branquióstegos y dos huesos medianos impares, el basihial y el urohial (Fig. 25B).

El hipohial superior (Fig. 36, arriba), de aspecto más o menos rectangular, está ubicado en la mitad superior de la concavidad formada por el ceratohial. Por debajo está articulado por sinartrosis con el hipohial inferior. Por el margen dorsal cóncavo y hacia el lado interno está ubicado entre los dos hipohiales, el primer basibranquial hacia atrás y el extremo posterior del basihial hacia adelante. Aproximadamente en su parte media, el hueso presenta un foramen para el paso de la arteria hioidea aferente proveniente del epi- y ceratohial. El ancho del hueso, en relación a su longitud total, es de $53 \%$ en Paralichthys isosceles, $66 \%$ en $P$. patagonicus y $80 \%$ en $P$. orbignyanus.

De forma triangular, el hipohial inferior presenta en su cara interna una concavidad ventral en la cual se aloja la rama antero-ventral del ceratohial. Por su borde dorsal está articulado con el hipohial superior a través de una delgada lámina de cartílago reforzada por pequeñas serraciones del hueso que interdigitan con sus correspondientes del hueso derecho. La altura máxima del hueso, en relación a su longitud máxima, es de $62 \%$ en $P$. orbignyanus, $58 \%$ en $P$. isosceles y $62,5 \%$ en $P$. patagonicus (Fig. 36, abajo). 


\section{PP}
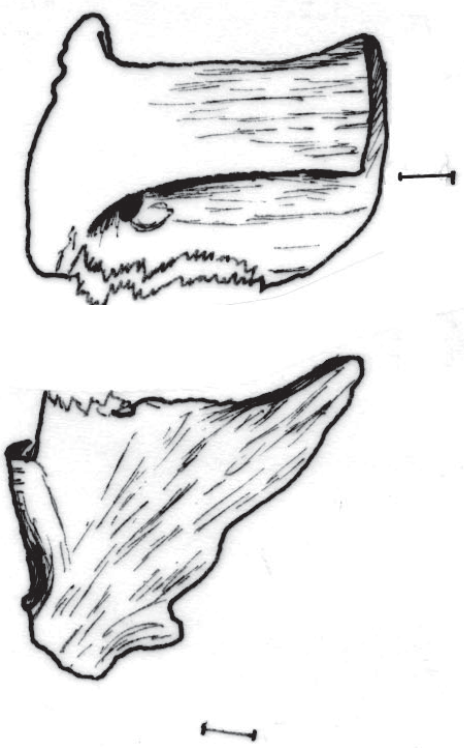

$\mathrm{PO}$
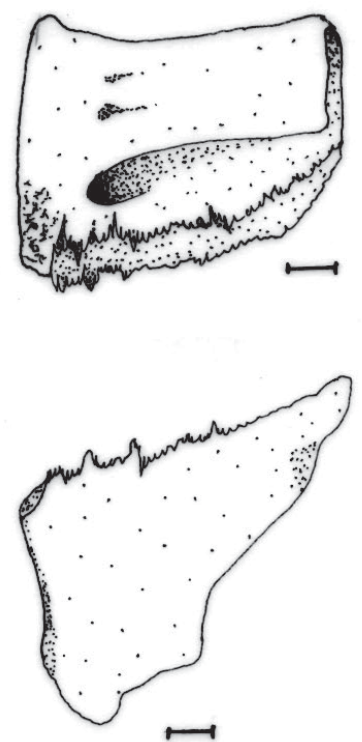

PI
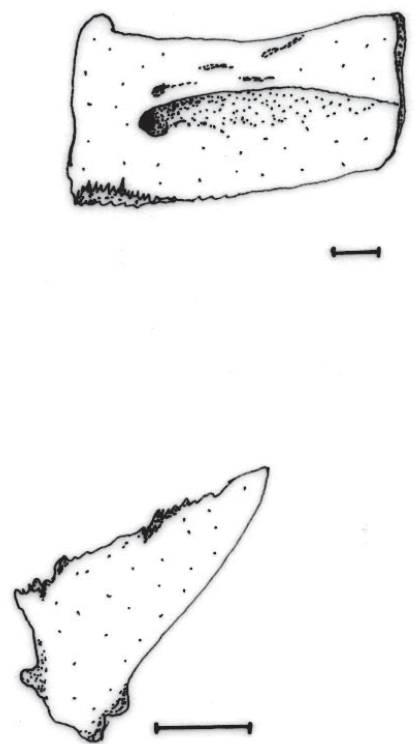

Fig. 36: Huesos hipohiales dorsal (arriba) y ventral (abajo) de Paralichthys patagonicus (PP), $P$. orbignyanus (PO) y $P$. isosceles (PI) en vista lateral. Escala $1 \mathrm{~mm}$.

Dorsal (upper) and vental (lower) hypohyals of Paralichthys patagonicus (PP), P. orbignyanus (PO) and P. isosceles (PI). Scale bar indicates $1 \mathrm{~mm}$.

El ceratohial es de forma semicircular, con una rama anteroventral a la cual se unen los hipohiales inferior y superior. Está perforado por una escotadura u orificio a través del cual pasa la arteria hioidea (Rojo 1988). Está articulado por sinartrosis dentada con el epihial por medio de una delgada lámina de cartílago y por una serie de procesos espinosos que interdigitan con sendos procesos provenientes del epihial. Por su parte ventral, el ceratohial soporta los cuatro primeros radios branquiostegos, de los cuales el primero y el cuarto se articulan por la cara externa del hueso, y el segundo y tercero están alojados en dos concavidades en el borde ventral del mismo (Fig. 37A). El margen dorsal del hueso es convexo en $P$. patagonicus y $P$. orbignyanus, mientras que en $P$. isosceles es comúnmente recto o cóncavo (Fig. 37B, C y D). La altura máxima del hueso (desde el borde dorsal al ventral), en relación a la longitud total (desde el extremo de la proyección anterior hasta el borde posteroventral, excluidos los procesos odontoides) es mayor en $P$. orbignyanus (rango $65-70 \%$; media $66 \%$ ) y menor en $P$. isosceles (47 - $53 \% ; 49 \%$ ), con valores intermedios para $P$. patagonicus $(60-63 \% ; 62 \%)$. El orificio que da paso a la arteria hioidea es más grande, en porcentaje del largo del hueso, en $P$. patagonicus $(29 \%)$ que en $P$. isosceles $(17 \%)$ y $P$. orbignyanus $(15 \%)$.

El epihial es un hueso de forma triangular con vértice dirigido hacia atrás y levemente inclinado hacia arriba. Dicha inclinación forma una pequeña concavidad donde está articulada por diartrosis el interhial. En la parte media del borde anterior, unas proyecciones digitiformes lo suturan fuertemente al ceratohial. Inferiormente, sobre la cara externa del hueso, están articulados el sexto y séptimo radio branquiostegos, mientras que el quinto radio lo hace en la zona de unión entre el epihial y el ceratohial (Fig. 37A). El borde dorsal del hueso presenta una ligera curvatura convexa en $P$. patagonicus, más pronunciada en $P$. isosceles y ausente en $P$. orbignyanus. En esta última especie dicho borde es recto, con una pequeña concavidad antes del cóndilo articular que sostiene al interhial. La cara externa del epihial está atravesada por dos canales, uno superior y otro inferior. El canal superior está ausente en $P$. isosceles y apenas marcado en $P$. orbignyanus (Fig. 38, arriba). 


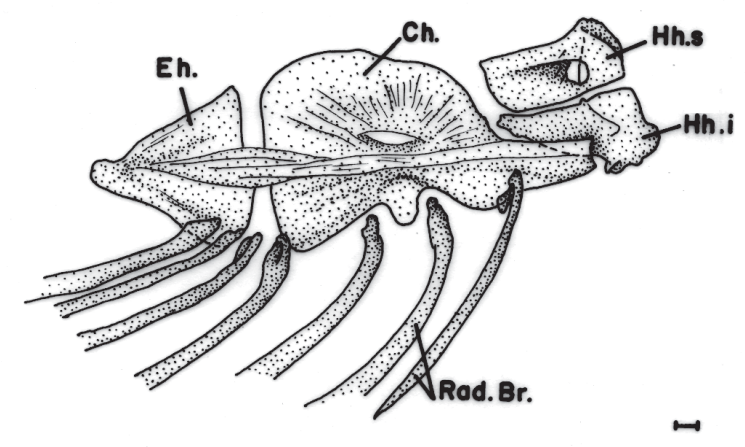

PP

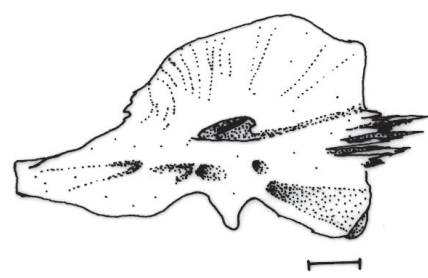

PI

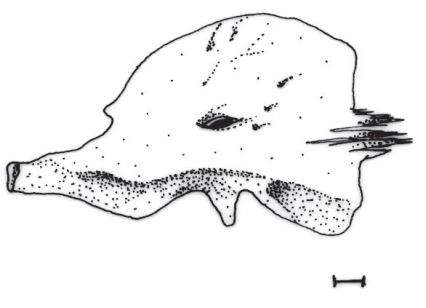

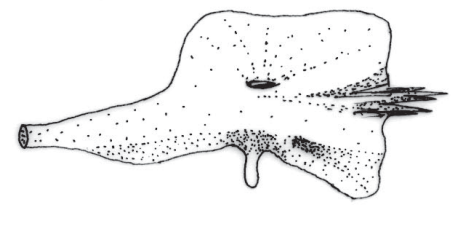

Fig. 37: Arco hioideo derecho de Paralichthys orbignyanus (A) y ceratohiales de Paralichthys patagonicus $(\mathrm{PP}), P$. orbignyanus $(\mathrm{PO})$ y $P$. isosceles $(\mathrm{PI})$ en vista lateral. Ver abreviaturas en el texto. Escala $1 \mathrm{~mm}$.

Right hyoid arc of Paralichthys orbignyanus (A) and certohyals of Paralichthys patagonicus (PP), P. orbignyanus (PO) and $P$. isosceles (PI) in lateral view. See abbreviations on text. Scale bar indicates $1 \mathrm{~mm}$.

El interhial, también llamado stilohial, es un pequeño hueso cilíndrico situado entre el extremo posterodorsal del epihial y la conexión cartilaginosa del simpléctico con el hiomandibular. Está unido, por medio de un fuerte ligamento, a la cara interna del preopérculo (Fig. 25B). El interhial en $P$. isosceles tiene una forma más globosa, tipo "botella", mientras que en $P$. patagonicus y $P$. orbignyanus, es de tipo bastoncito. La cara externa del hueso presenta en $P$. isosceles un canal bien notorio, mientras que en $P$. patagonicus dicho canal es menos notable y está ausente en $P$. orbignyanus (Fig. 38, medio). El ancho del hueso en relación a su largo es de 28 $37 \%$ en $P$. isosceles, 36 - $38 \%$ en $P$. orbignyanus y $38-41 \%$ en $P$. patagonicus.

El número de radios branquiostegos es de siete en las tres especies e incrementan su tamaño de adelante hacia atrás. Cuatro están articulados al ceratohial, dos al epihial y uno en el espacio entre estos dos huesos (Fig. 37A). No se verificaron diferencias morfológicas en ninguna de las especies estudiadas.
El basihial o glosohial es un pequeño hueso edéntulo en forma de espátula que forma el esqueleto de la lengua. Su extremo anterior es suavemente redondeado en $P$. patagonicus y romo en las otras dos especies de Paralichthys estudiadas. Posteriormente se une por medio de un disco cartilaginoso con el primer basibranquial. Queda encerrado, hacia los costados, por los hipohiales superiores. No hubieron diferencias entre los huesos de las tres especies.

El urohial tiene forma de "boomerang" con vértice dirigido hacia atrás. Los dos brazos anteriores presentan una proyección membranosa. La rama superior está unida, a través de fuertes ligamentos, al glosohial, primer basibranquial e hipohiales superior e inferior (Fig. 40). La rama inferior llega hasta los primeros radios branquiostegos. Posteriormente el urohial se desdobla en dos expansiones laminares laterales que se sitúan delante del borde anterior de la rama inferior de los cleitra. Amaoka (1969) considera al urohial constituido por las siguientes partes: una principal en forma de varilla y rodeada por 
PP

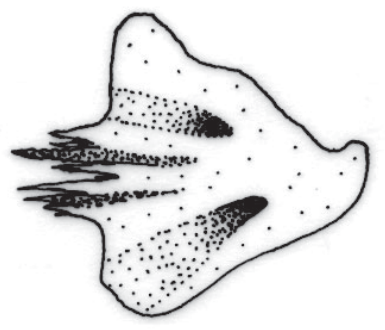

$\mapsto$
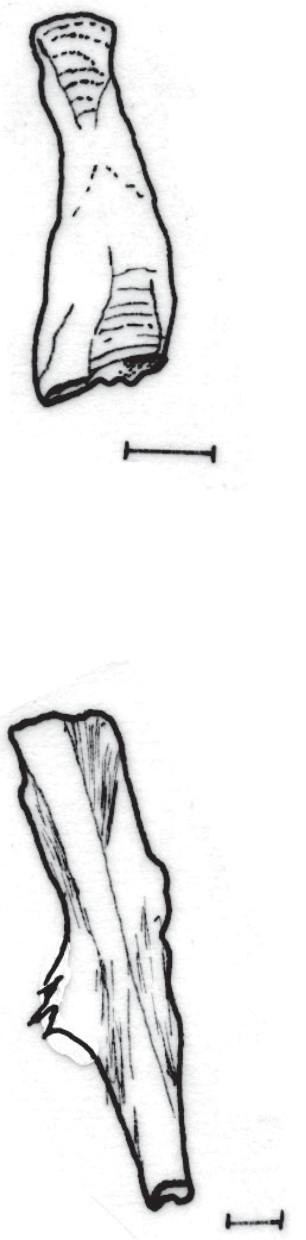

PO
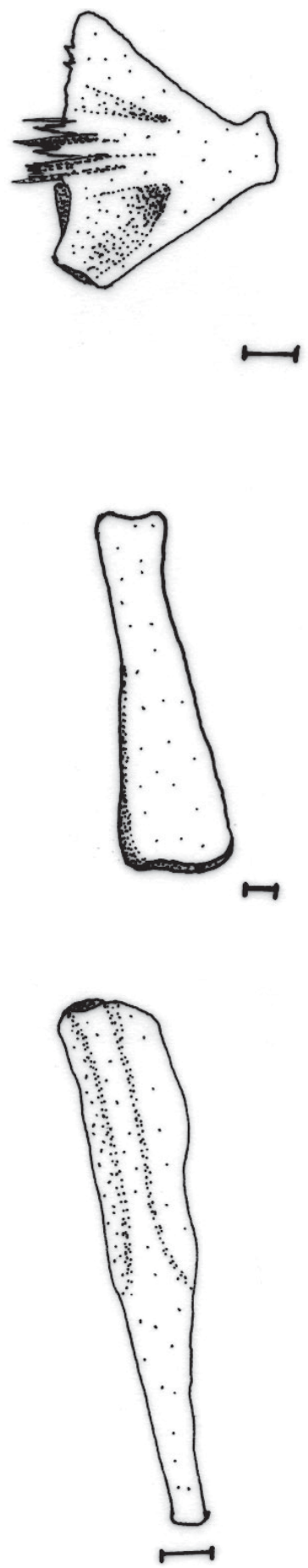

PI

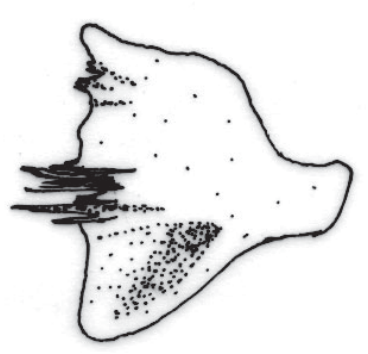

$\longrightarrow$
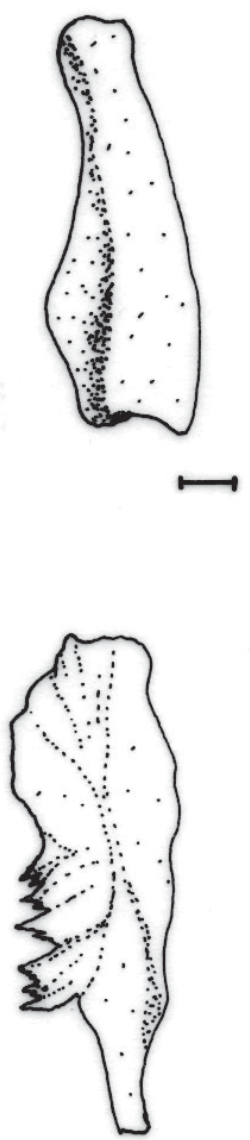

Fig. 38: Huesos epihial (arriba), interhial (medio) y simpléctico (abajo) de Paralichthys patagonicus (PP), P. orbignyanus (PO) y $P$. isosceles (PI) en vista lateral. Escala $1 \mathrm{~mm}$.

Epihyal (upper), interhyal (middle), and symplectic (lower) bones of Paralichthys patagonicus (PP), P. orbignyanus (PO) and $P$. isosceles $(\mathrm{PI})$ in lateral view. Scale bar indicates $1 \mathrm{~mm}$. 
una fina membrana (en nuestro caso, las dos ramas superior e inferior con las expansiones laminares correspondientes); una apófisis cardíaca (vértice posterior) y una parte sciática, conectada supraposteriormente con el cleitrum por una delgada membrana (parte laminar de la rama inferior). Las siguientes son las diferencias morfológicas y morfométricas observadas entre las tres especies de Paralichthys analizadas (Fig. 39, abajo): (1) en $P$. isosceles las dos ramas (superior e inferior) se aproximan entre sí delimitando un ángulo de 25 a $28^{\circ}$. En $P$. patagonicus y $P$. orbignyanus ese ángulo es de 47 a $50^{\circ}$; (2) la expansión laminar del brazo superior finaliza en el extremo anterior de dicha rama en $P$. isosceles, mientras que en $P$. patagonicus y $P$. orbignyanus ese extremo queda libre de tal expansión; (3) el borde dorsal de la lámina ósea es convexo anteriormente en las tres especies, con márgenes aserrados en $P$. patagonicus y $P$. orbignyanus, y lisos en $P$. isosceles; (4) la rama inferior presenta un canal longitudinal en $P$. isosceles; está menos marcado en $P$. patagonicus y ausente en $P$. orbignyanus; (5) la longitud de la rama inferior es un $78 \%$ la longitud de la superior en $P$. orbignyanus, un $83 \%$ en $P$. isosceles y un $84 \%$ en $P$. patagonicus; (6) la altura máxima de la rama superior, incluyendo la expansión ósea, en relación a la longitud de dicha rama fue de 25 - $27 \%$ (media $26 \%$ ) en $P$. patagonicus, $29-32 \%$ (31\%) P. orbignyanus y $32-40 \%(37 \%)$ en $P$. isosceles.

\section{PP}
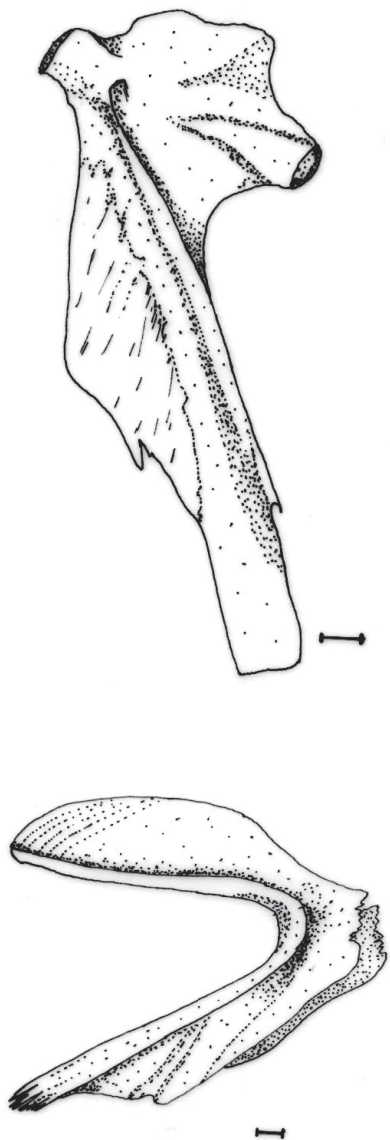
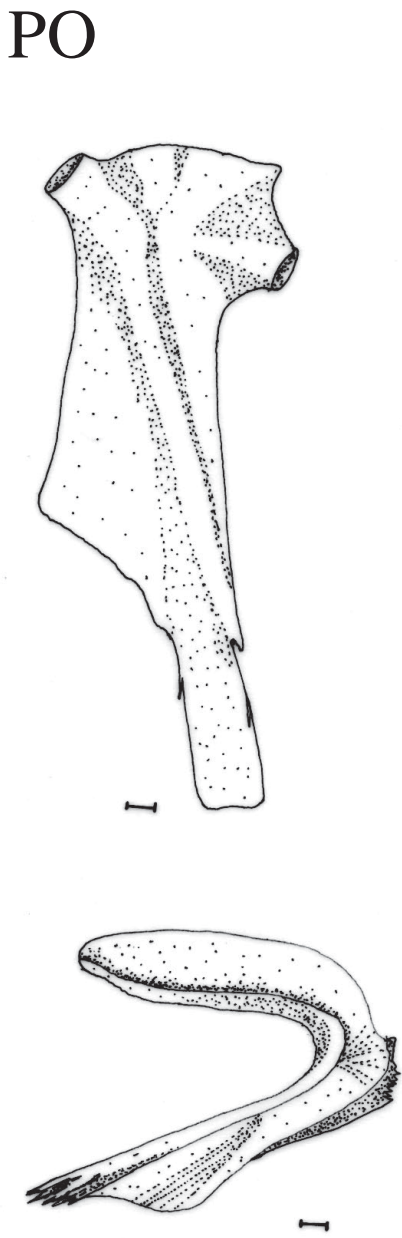

PI
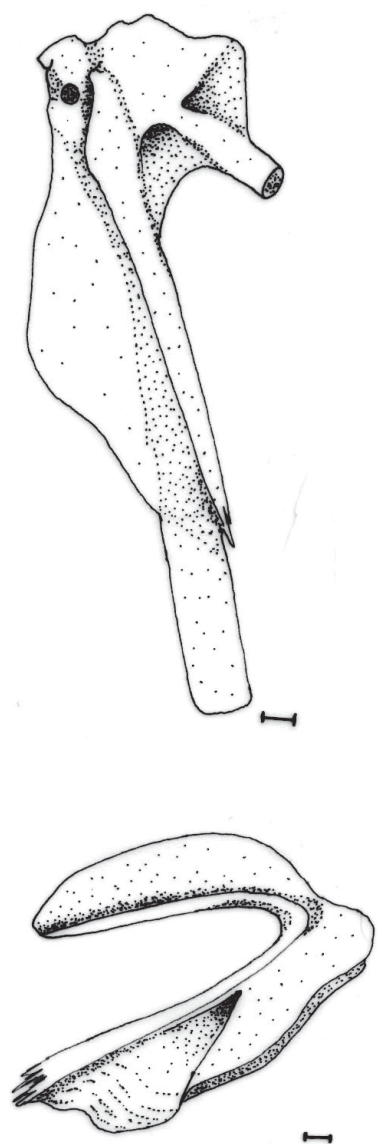

Fig. 39: Huesos hiomandibular (arriba) y urohial (abajo) de Paralichthys patagonicus (PP), $P$. orbignyanus $(\mathrm{PO})$ y $P$. isosceles $(\mathrm{PI})$ en vista lateral. Escala $1 \mathrm{~mm}$.

Hyomandibula (upper) and urohyal (lower) bones of Paralichthys patagonicus (PP), P. orbignyanus (PO) and $P$. isosceles (PI) in lateral view. Scale bar indicates $1 \mathrm{~mm}$. 


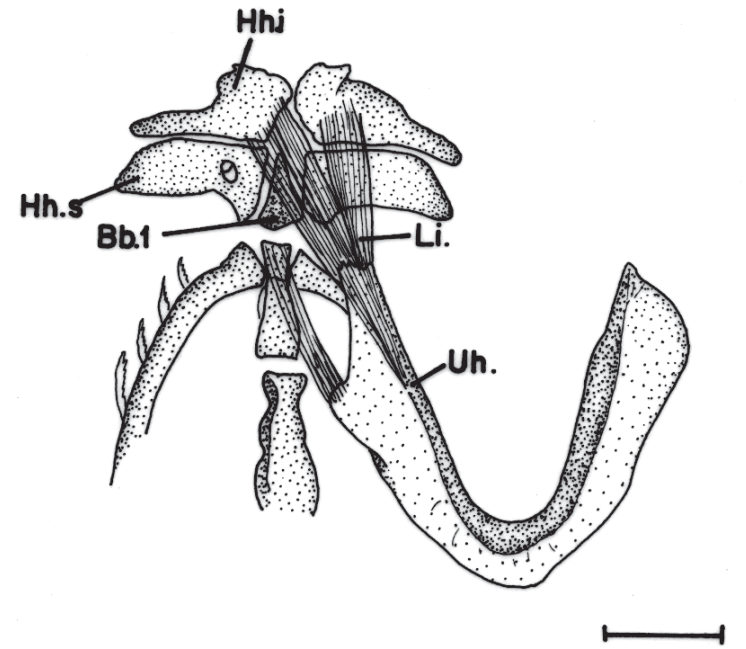

Fig. 40: Uniones ligamentarias del brazo inferior del urohial de Paralichthys isosceles en vista ventral. Escala $1 \mathrm{~mm}$.

Joints by ligaments of the lower arm of the urohyal of Paralichthys isosceles in ventral view. Scale bar indicates $1 \mathrm{~mm}$.

\section{Aparato opercular}

La serie está constituida por cuatro huesos comprimidos lateralmente que cubren y protegen los arcos branquiales. Todos ellos son pares y de origen dérmico (preopérculo, opérculo, subopérculo e interopérculo).

El preopérculo presenta una forma de media luna o en L, con la rama superior más prolongada. Tanto el derecho como el izquierdo son levemente asimétricos. Las ramas superior e inferior son un poco más largas en el preopérculo izquierdo (lado oculado). Asimismo el preopérculo del lado ciego es un poco más ancho que el del lado oculado. Esta asimetría está más marcada en $P$. isosceles (Fig. 41C, arriba). Todo el borde anterior de la rama superior está insertado en un surco posterolateral del proceso simpléctico del hiomandibular. El borde anterior de la rama inferior está articulado por sincondrosis con el margen posterior del cuadrado. Todo el borde ventral del hueso se superpone al margen dorsal del interopérculo. El extremo anterior se une al anguloarticular por medio de un ligamento. Por el lado interno y justo en el ángulo de unión de las ramas superior e inferior, se une al interhial a través de un fuerte ligamento. No se encontraron diferencias morfológicas en los huesos de las tres especies (Fig. 41, arriba).
El opérculo es sumamente delgado, laminar en toda su extensión. En la parte dorsoanterior presenta un cóndilo de articulación con el proceso posterior del hiomandibular. Ventralmente se contacta por sinartrosis escamosa con el subopérculo y posteriormente constituye el margen de apertura a la cámara branquial. Woolcott et al. (1968) encontraron diferencias en la forma del opérculo, hecho que les resultó útil para separar las especies del género Paralichthys por ellos estudiadas. Se encontraron las siguientes diferencias (Fig. 41, medio): (1) en $P$. patagonicus, el borde superior del opérculo presenta una muesca o escotadura en la zona de unión de la parte laminar del hueso con el cóndilo articular del hiomandibular. Dicha concavidad está ligeramente presente en $P$. orbignyanus y ausente en $P$. isosceles; (2) en $P$. patagonicus y $P$. orbignyanus la forma del hueso es más o menos triangular, mientras que en $P$. isosceles es cuadrangular; (3) el ancho máximo del hueso en relación a su longitud es de $63-70 \%$ (media $65 \%$ ) en $P$. orbignyanus, $63-73 \%(68 \%)$ en $P$. isosceles y de $64-71 \%$ (69\%) en P. patagonicus.

El subopérculo es un hueso delgado y laminar, de forma oval. Presenta un proceso posterodorsal, delgado y largo que recorre todo el margen ventral del opérculo. Por su borde anterior se relaciona con el interopérculo con el cual está unido por medio de una membrana. Si bien Woolcott et al. (1968) establecen diferencias específicas en la longitud del proceso membranoso del subopérculo, no pudo tomarse en cuenta debido al proceso de cocción para la preparación del esqueleto que distorsionó el frágil proceso. Se registró una asimetría respecto al ancho de los huesos derecho e izquierdo. El del lado derecho resultó ser un $11 \%$ más ancho que el izquierdo en las tres especies. Las diferencias interespecíficas observadas fueron (Fig. 41, abajo): (1) el proceso medio anterior que se une por cartílago con el preopérculo está muy desarrollado y emarginado del cuerpo laminar del subopérculo en $P$. orbignyanus. En $P$. patagonicus la emarginación mencionada es menor, y mucho menor aún en $P$. isosceles. En las primeras dos especies el borde ventral del proceso anterior conforma con el margen ventroanterior del subopérculo una concavidad a la cual se une con el borde superior del interopérculo. Esa concavidad está ausente en $P$. isosceles. (2) La 


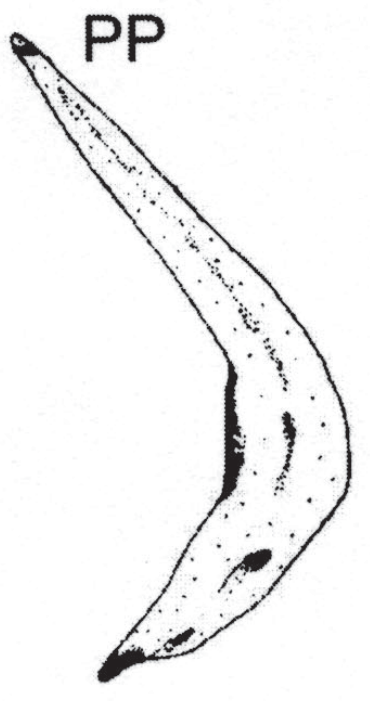

$\mathrm{PO}$
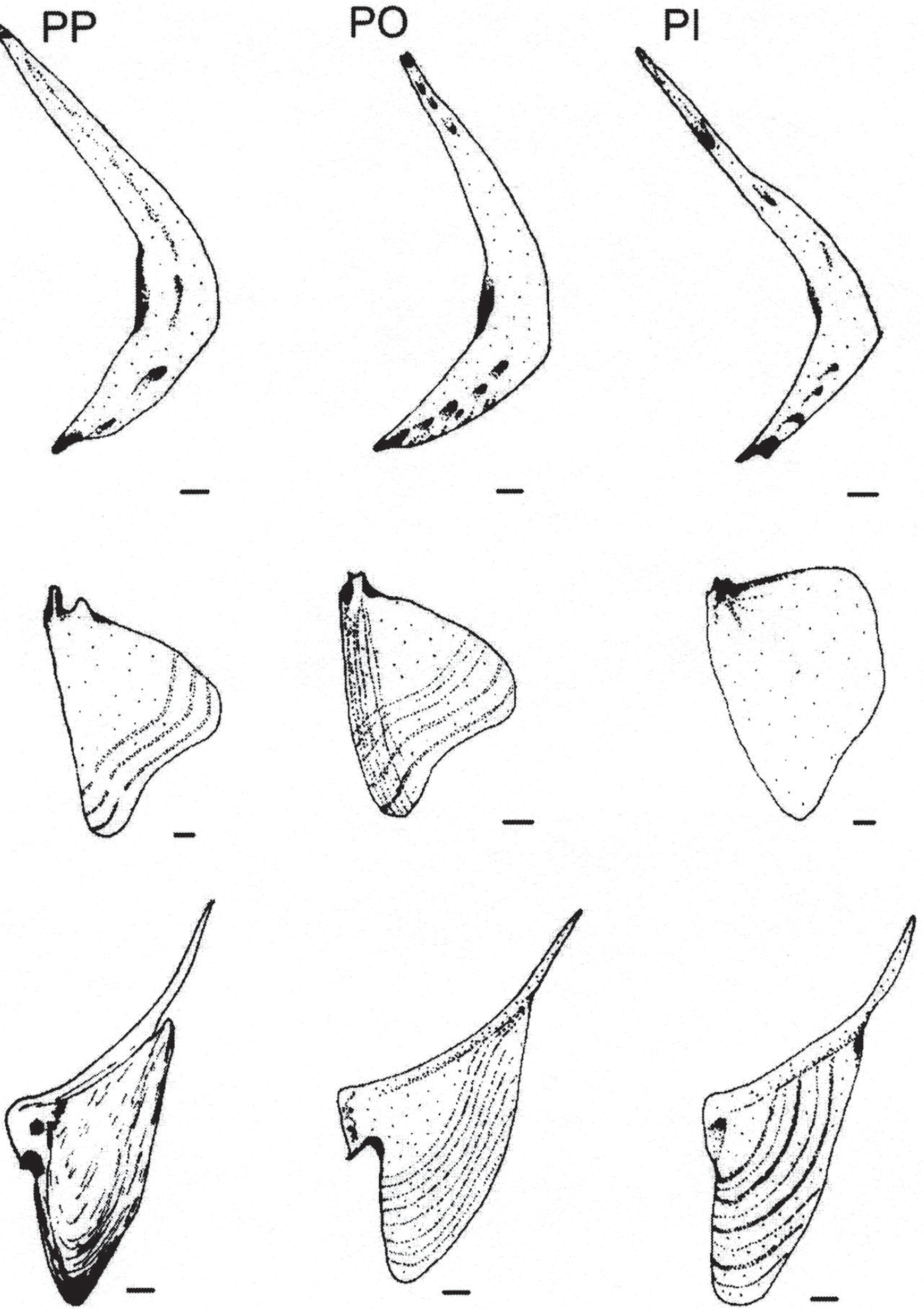

Fig. 41: Huesos preopercular (arriba), opercular (medio) y subopercular (abajo) de Paralichthys patagonicus $(\mathrm{PP}), P$. orbignyanus (PO) y $P$. isosceles $(\mathrm{PI})$ en vista lateral. Escala $1 \mathrm{~mm}$.

Peopercle (upper), opercle (middle) and subopercle (lower) bones of Paralichthys patagonicus (PP), P. orbignyanus (PO) and $P$. isosceles $(\mathrm{PI})$ in lateral view. Scale bar indicates $1 \mathrm{~mm}$. 
longitud del proceso medio anterior, en relación a la longitud del subopérculo fue menor en $P$. orbignyanus (rango $18-23 \%$; media $20 \%$ ), mayor en $P$. isosceles (26 - $32 \%$; $29 \%)$ y con valores intermedios en $P$. patagonicus (17 - $25 \%$; $23 \%$ ). (3) La relación ancho/largo del subopérculo, indica que $P$. isosceles presenta un subopérculo más estrecho $(31 \%)$ que en las otras dos especies $(36 \%)$.

El interopérculo es el hueso menos laminar de la serie. Su borde anterior está cubierto levemente por la rama inferior del preopérculo, al cual se une por medio de tejido conectivo. Su borde superior cóncavo encaja perfectamente en una convexidad del subopérculo. Por su cara interna un fuerte ligamento une el borde anterior del interopérculo con el epihial. Abundante tejido conectivo relaciona a este hueso con el interhial y ceratohial. Coincidentemente con Cervigón (1985), no se halló ninguna sutura entre el interopérculo y el epihial, como mencionan Futch et al. (1972: 45) para Achirus lineatus. La única relación existente se manifestó a través de tejido conectivo. Inferiormente, un fuerte ligamento une el interopérculo con el retroarticular (Fig. 26). Se observó una asimetría entre los huesos izquierdo y derecho en las tres especies. Mientras que en $P$. isosceles el interopérculo del lado oculado fue un $10,4 \%$ más largo que su correspondiente al lado ciego, en $P$. orbignyanus fue del $4,5 \%$ y en $P$. patagonicus del 5,5\%. La anchura del hueso cabe dos veces en su longitud en $P$. isosceles, 1,4 veces en $P$. orbignyanus y 1,7 en $P$. patagonicus (Fig. 42).

\section{Arcos branquiales}

Los cinco arcos branquiales dan sostén a las branquias, músculos respiratorios, branquispinas y dientes faríngeos. La serie está compuesta de cuatro basibranquiales, tres pares de hipobranquiales, cinco pares de ceratobranquiales, cuatro pares de epibranquiales y cuatro pares de faringobranquiales (Fig. 43). Amaoka (1969) denomina al quinto par de ceratobranquiales como huesos faríngeos inferiores y a los faringobranquiales como huesos faríngeos superiores.

\section{PP}

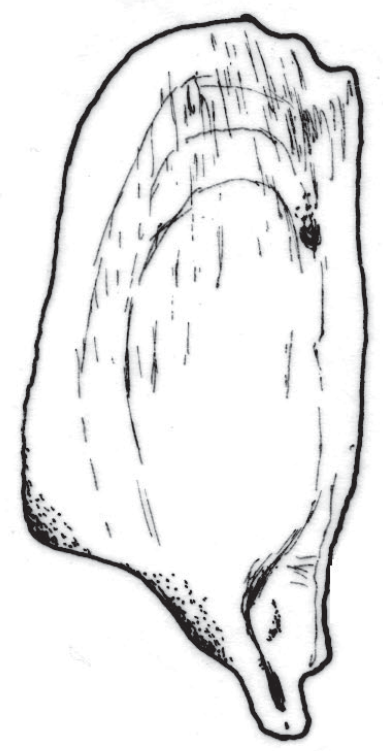

PO

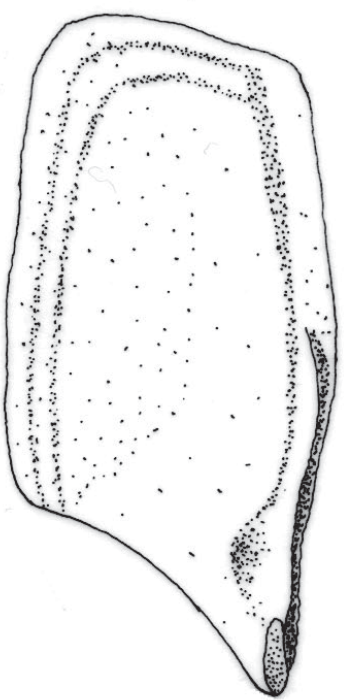

PI

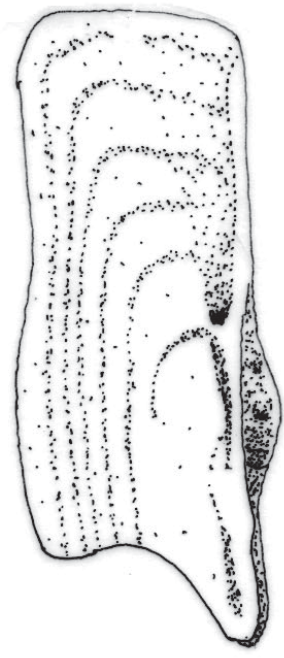

Fig. 42: Interopérculos de Paralichthys patagonicus (PP), P. orbignyanus (PO) y P. isosceles (PI) en vista lateral. Escala $1 \mathrm{~mm}$.

Interopercles of Paralichthys patagonicus (PP), P. orbignyanus (PO) and P. isosceles (PI) in lateral view. Scale bar indicates $1 \mathrm{~mm}$. 


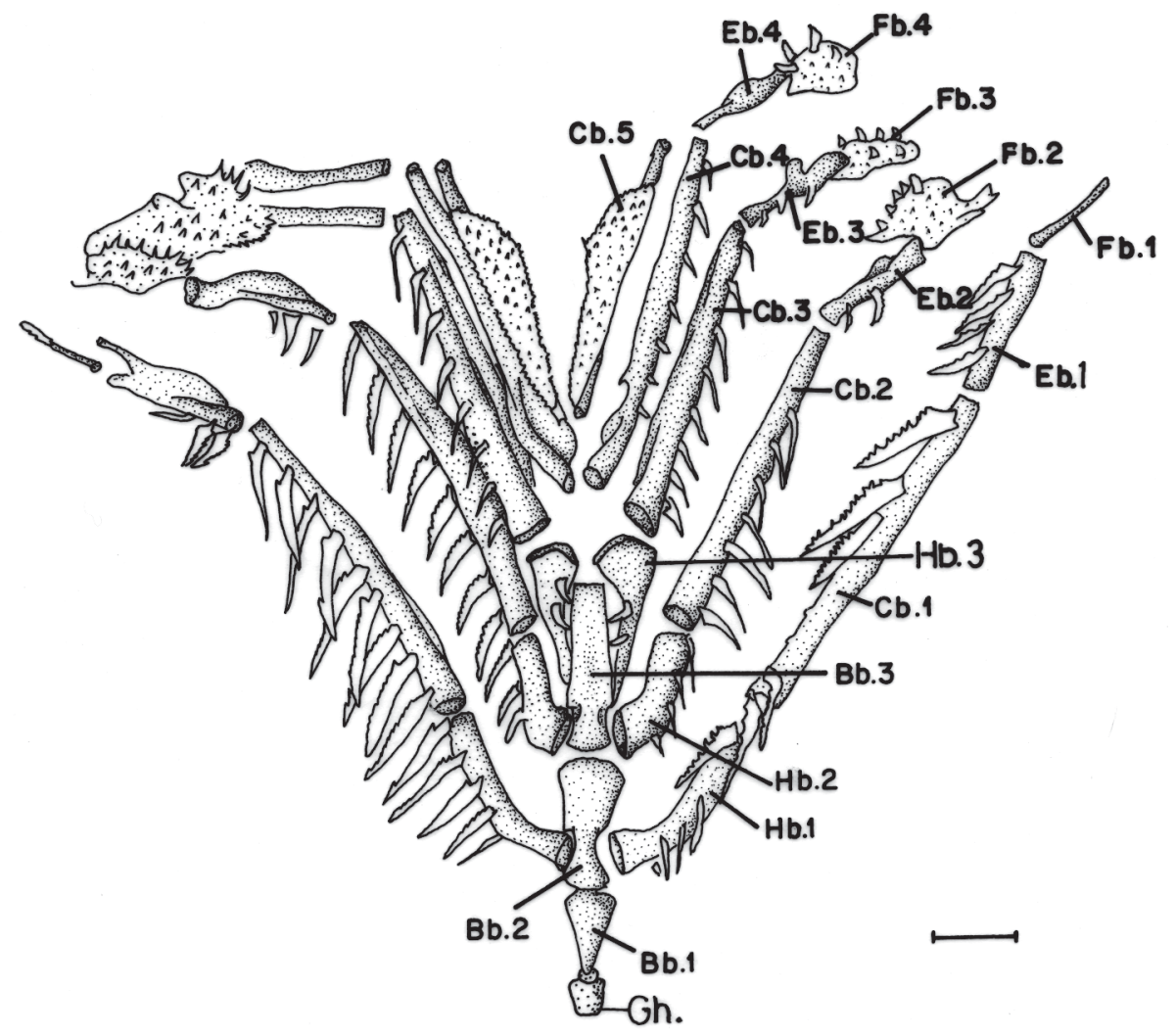

Fig. 43: Arcos branquiales de Paralichthys orbignyanus en vista dorsal. Escala $1 \mathrm{~mm}$. Branchial arches of Paralichthys orbignyanus in dorsal view. Scale bar indicates $1 \mathrm{~mm}$.

Los cuatro basibranquiales están situados en la línea media del piso de la faringe. El primero se ubica entre el basihial adelante, y el segundo basibranquial atrás, con el cual está relacionado a través de una sutura. Los hipohiales lo encierran por el costado. Unos fuertes ligamentos unen el primer basibranquial con los hipohiales inferiores y con el urohial. En las tres especies examinadas la forma de este hueso resultó ser triangular con la base articulada con el segundo basibranquial y el vértice dirigido anteriormente (Fig. 43). Un pequeño proceso redondeado dirigido dorsalmente caracteriza al extremo anterior. Dicho proceso está más desarrollado en $P$. patagonicus que en las otras especies. El segundo basibranquial está relacionado lateralmente con los extremos redondeados de los primeros hipobranquiales. La forma del hueso es aproximadamente rectangular en vista dorsal con una leve constricción cerca del extremo anterior (Fig. 43). El tercer basibranquial está ubicado por detrás del segundo. A los costados está relacionado con los segundos y terceros hipobranquiales. El cuarto basibranquial es cartilaginoso.

Los primeros hipobranquiales son huesos robustos, largos, ensanchados anteriormente. Están articulados con las concavidades laterales del segundo basibranquial. Hacia atrás están articulados con los primeros ceratobranquiales. En su superficie dorsal llevan branquispinas (tres a cuatro en $P$. patagonicus y $P$. isosceles; cinco a seis en $P$. orbignyanus). Paralichthys patagonicus presenta un hipobranquial relativamente más corto (33\% de la longitud total de la rama inferior del primer arco) que $P$. orbignyanus (35\%) y $P$. isosceles (43\%). Los segundos hipobranquiales son huesos más cortos que los primeros. Por su extremo anterior están articulados en unas concavidades laterales de la parte anterior del tercer basibranquial. Posteriormente están relacionados con los segundos ceratobranquiales. También llevan 
branquispinas; el rango es de dos a tres para $P$. isosceles y $P$. patagonicus, y cuatro para $P$. orbignyanus. No se observaron diferencias en la morfología y morfometría entre los hipobranquiales de las tres especies. Los terceros hipobranquiales están situados a ambos lados del tercer basibranquial. Presentan una delgada proyección anterior que está prolongada hasta la parte anterior y ventral del tercer basibranquial. Posteriormente se caracterizan por poseer una amplia expansión ósea que está articulada con los terceros ceratobranquiales. Si bien no se observaron diferencias morfológicas entre los huesos de las tres especies, en $P$. isosceles los terceros hipobranquiales están tapizados dorsalmente por un parche de diminutos dentículos dérmicos (Fig. 44C), ausente en las otras especies y reemplazados por pequeñas branquispinas cuyo número va de uno en $P$. patagonicus (Fig. 45) a tres en $P$. orbignyanus (Fig. 43).

Los ceratobranquiales primero, segundo $\mathrm{y}$ tercero son los huesos más largos de la serie branquial. Están articulados, por medio de puentes cartilaginosos, con sus respectivos hipobranquiales anteriormente y epibranquiales posteriormente. Los primeros cuatro pares están provistos de branquispinas. El número, en el primer ceratobranquial, varía según la especie. En $P$. isosceles es de cinco a seis, en $P$. patagonicus de siete a ocho y en $P$. orbignyanus de nueve a diez. El quinto par de ceratobranquiales está provisto de un parche de diminutos dentículos en $P$. patagonicus y $P$. orbignyanus (Fig. 43 y 45). En $P$. isosceles, existen dientes más prominentes, caniniformes (Fig. 44D). Esta serie de dientes constituye los dientes faríngeos inferiores.

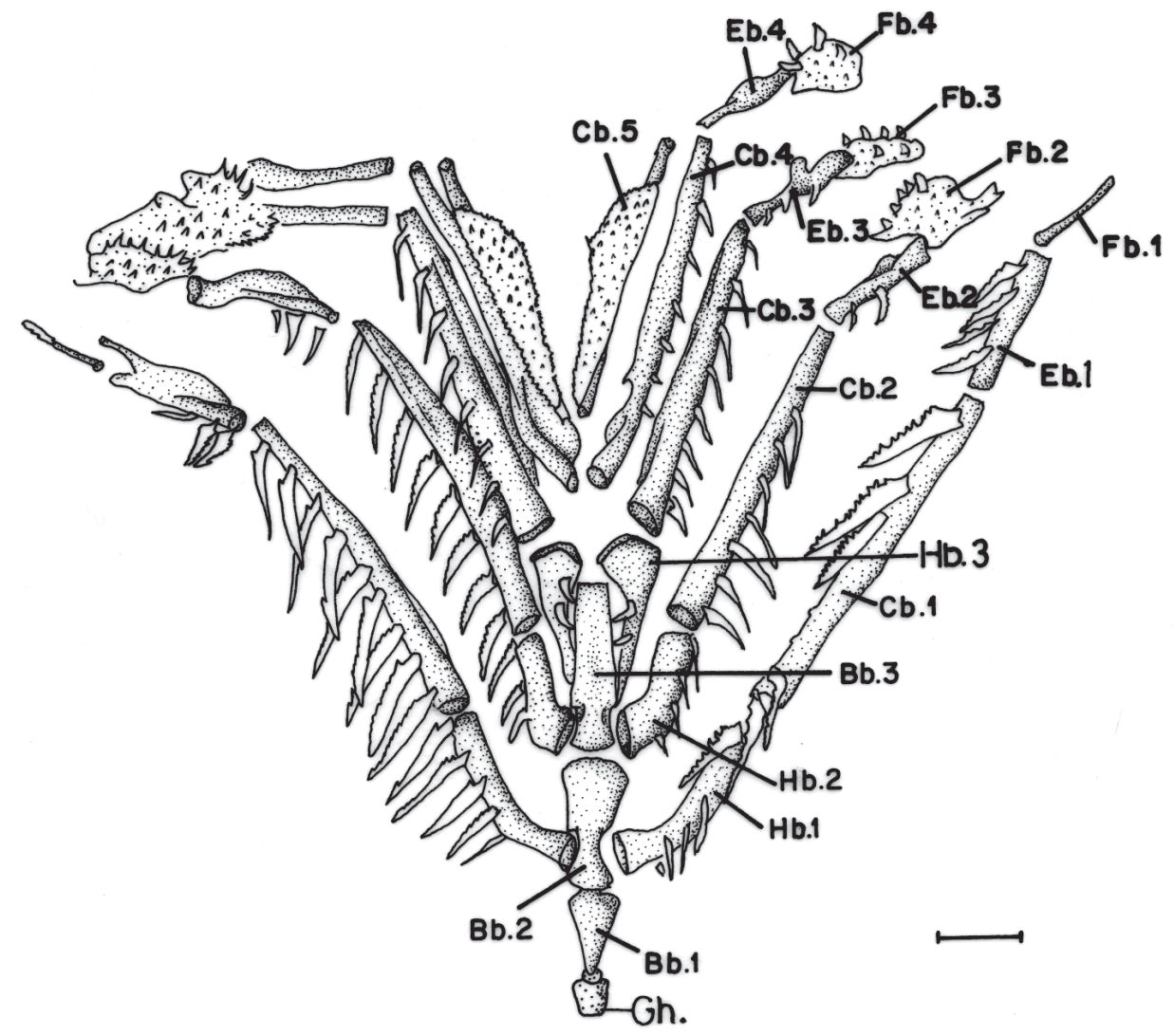

Fig. 44: Primer (A), segundo (B), tercer (C), cuarto y quinto (D) arcos branquiales izquierdos de Paralichthys isosceles en vista lateral. Ver abreviaturas en el texto. Escala $1 \mathrm{~mm}$.

First (A), second (B), third (C), fourth and fifth (D) left branchial arches of Paralichthys isosceles in lateral view. See abbreviations on text. Scale bar indicates $1 \mathrm{~mm}$. 


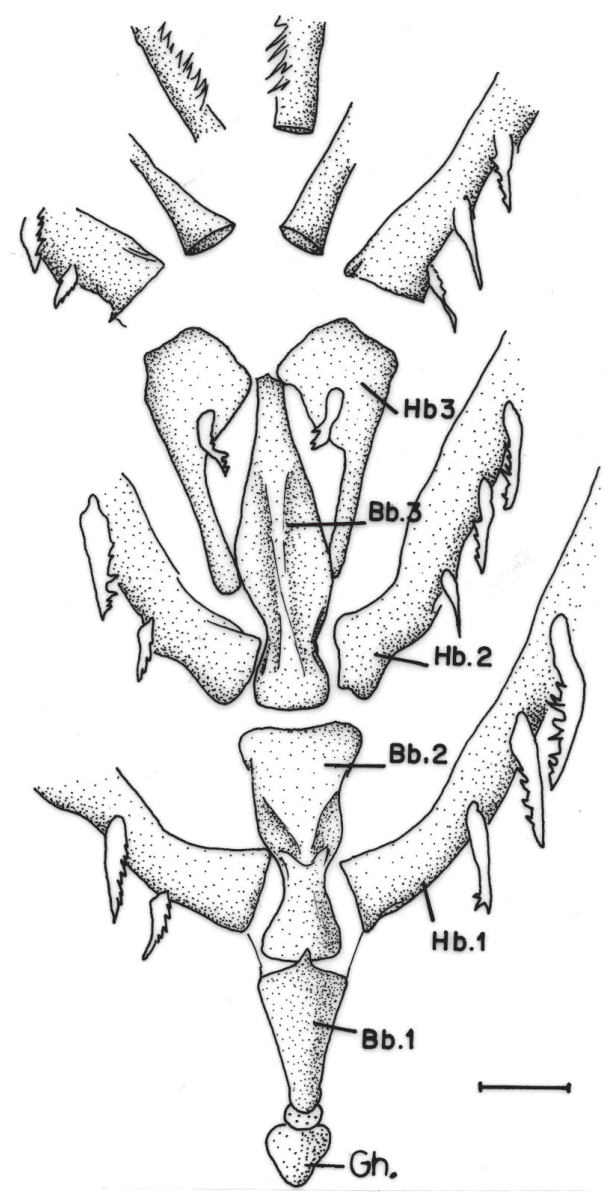

Fig. 45: Arcos branquiales (parte inferior) de Paralichthys patagonicus en vista dorsal. Ver abreviaturas en el texto. Escala $1 \mathrm{~mm}$.

Branchial arches (lower part) of Paralichthys patagonicus in dorsal view. See abbreviations on text. Scale bar indicates $1 \mathrm{~mm}$.

Los cuatro pares de epibranquiales están articulados anteriormente con sus respectivos ceratobranquiales y posteriormente con sus correspondientes faringobranquiales. Constituyen la rama superior de los arcos branquiales. En su superficie dorsal llevan branquispinas. El número, en el primer arco, es de uno a dos en $P$. isosceles, dos a tres en $P$. patagonicus y cuatro a cinco en $P$. orbignyanus. En las dos primeras especies, también se observó la presencia de tubérculos o mamelones cuyo número fue variable. En las tres especies analizadas la forma del primer epibranquial resultó ser aproximadamente la misma, con una bifurcación o proceso uncinado en el extremo de articulación con el faringobranquial y con una expansión laminar que se extiende desde el extremo de una de las bifurcaciones a todo lo largo del hueso. El segundo epibranquial presenta forma de barra con un proceso laminar en la parte media del hueso. El tercer epibranquial es un hueso robusto, corto, en forma de horqueta, con un proceso uncinado presente en las tres especies estudiadas (Fig. 40B y 41). En $P$. patagonicus el proceso posterior es espinoso y el hueso lleva en su cara interna un parche de dientes faríngeos superiores pequeños (Fig. 43). En P. orbignyanus, el proceso tiene las mismas características que en $P$. patagonicus, pero existe una expansión laminar que une dicho proceso con el eje principal del epibranquial (Fig. 45), característica ausente en $P$. patagonicus. En $P$. isosceles el proceso posterior es mucho más amplio y redondeado y los dientes faríngeos superiores más conspicuos (Fig. 44C). El cuarto epibranquial no lleva dientes. La forma del hueso es curva con una expansión lateral y laminar presente en la parte media del hueso en las tres formas analizadas.

Los faringobranquiales son huesos bastante irregulares entre sí. Todos, excepto el primero, portan dientes muy agudos que constituyen los dientes faríngeos superiores. El primer faringobranquial es corto y muy delgado. Su extremo superior se sitúa en el neurocráneo, a nivel del proótico. Los otros faringobranquiales están relacionados con la parte basal y posterior del neurocráneo a través de nódulos cartilaginosos. El segundo faringobranquial está contactado con su correspondiente epibranquial por la superficie inferior y con el tercer faringobranquial por una amplia superficie lateral. El tercer hueso faringobranquial es el más grande del grupo, contactado inferiormente con su respectivo epibranquial, a sus lados con el segundo faringobranquial anteriormente y cuarto faringobranquial posteriormente. El cuarto faringobranquial es muy pequeño. Está relacionado lateralmente con el tercer faringobranquial e inferiormente con el cuarto epibranquial (Fig. 43 a 45). En P. patagonicus y $P$. orbignyanus hay mayor desarrollo de los dientes faríngeos superiores del tercero y cuarto faringobranquiales. En $P$. isosceles el desarrollo es el mismo en todos los faringobranquiales.

En la Tabla 1 se resumen las diferencias y semejanzas más conspicuas de aquellos componentes óseos del cráneo de las tres especies del género Paralichthys en aguas del Atlántico suroccidental. 
TABLA 1

Caracteres osteológicos diagnósticos del esqueleto craneal de tres especies del género Paralichthys

Osteological diagnostic features of the cranial skeleton of three species of Paralichthys

\begin{tabular}{|c|c|c|c|}
\hline Hueso & P.patagonicus & P. orbignyanus & P. isosceles \\
\hline $\begin{array}{l}\text { Paretmoides } \\
\text { derecho }\end{array}$ & $\begin{array}{l}\text { Dos procesos posteriores espino- } \\
\text { sos. Borde superior cóncavo }\end{array}$ & $\begin{array}{l}\text { Dos procesos posteriores espino- } \\
\text { sos. Borde superior cóncavo }\end{array}$ & $\begin{array}{l}\text { Tres procesos posteriores espi- } \\
\text { nosos. Borde superior convexo }\end{array}$ \\
\hline $\begin{array}{l}\text { Lacrimal iz- } \\
\text { quierdo }\end{array}$ & $\begin{array}{l}\text { Brazo inferior poco más largo que } \\
\text { el superior. Leve concavidad en } \\
\text { cara externa. Brazo inferior recto }\end{array}$ & $\begin{array}{l}\text { Brazo inferior el doble de largo } \\
\text { que el superior. Cara externa sin } \\
\text { concavidad. Brazo inferior recto }\end{array}$ & $\begin{array}{l}\text { Brazo inferior el doble de largo } \\
\text { que el superior. Cara externa con } \\
\text { profunda concavidad. Brazo in- } \\
\text { ferior curvo }\end{array}$ \\
\hline $\begin{array}{l}\text { Serie infraor- } \\
\text { bitaria del } \\
\text { lado ciego }\end{array}$ & 4 a $5+1$ & $6+1$ & 4 a $6+1$ \\
\hline $\begin{array}{l}\text { P t e r ó t i c o } \\
\text { (longitud pro- } \\
\text { ceso anterior) }\end{array}$ & $40 \%$ del largo del hueso & $44 \%$ del largo del hueso & $31 \%$ del largo del hueso \\
\hline $\begin{array}{l}\text { Otolito iz- } \\
\text { quierdo }\end{array}$ & $\begin{array}{l}\text { Subtriangular con rostro angulo- } \\
\text { so y romo. Cara interna leve- } \\
\text { mente convexa con surco pro- } \\
\text { fundo. Borde dorsal liso }\end{array}$ & $\begin{array}{l}\text { Más o menos cuadrangular con } \\
\text { rostro anguloso y redondeado. } \\
\text { Cara interior convexa con surco } \\
\text { profundo. Borde dorsal irregular }\end{array}$ & $\begin{array}{l}\text { Más o menos cuadrangular con } \\
\text { rostro prominente y redondeado. } \\
\text { Cara interna levemente convexa } \\
\text { con surco no profundo. Borde } \\
\text { dorsal liso }\end{array}$ \\
\hline
\end{tabular}

Otolito dere- Subtriangular. Cara interna levecho

Cresta supraoccipital

Premaxilar (proceso) ascendente)

Maxilar

Dentario

Anguloarticular (longitud del canal óseo)

Palatino izquierdo (anchura del ex$\mathrm{t} \quad \mathrm{r} \quad \mathrm{e} \quad \mathrm{m}$ o superior)

Ectopterigoides (proceso laminar posterior)

Mesopterigoides (borde posterior)

mente convexa. Borde posterior con escotadura media. Borde ventral liso

Borde posterior recto. Proces premaxilar con leve concavidad

Poro oval ocupa un $17 \%$ la longitud del hueso. Seis a nueve dientes $($ media $=7,4)$

$30 \%$ del largo del hueso

Aserrado con proyección espinosa
Oblongo. Cara interna bien convexa. Borde posterior con escotadura media. Borde ventral festoneado o irregular

Baja y ubicada en la línea media del hueso. Con otra pequeña quilla media dorsal

Largo y situado bien debajo del extremo anterior del hueso

Borde posterior recto. Proceso premaxilar liso

Poro oval ocupa un $11 \%$ de la longitud del hueso. Nueve a 13 dientes $($ media $=11)$

$38 \%$ del largo del hueso

Aproximadamente cuadrangular. Cara interna levemente convexa. Borde posterior sin escotadura media. Borde ventral liso

Alta y desplazada hacia la derecha. Sin quilla media dorsal

Corto y situado cerca del extremo anterior del hueso

Borde posterior oblicuo. Proceso premaxilar con fosa

Poro oval ocupa un $20 \%$ de la longitud del hueso. 16 a 21 dientes $($ media $=18,5)$

$21 \%$ del largo del hueso

$22 \%$ del largo del hueso

$12 \%$ del largo del hueso

Con bordes lisos

Con bordes aserrados

Liso con leve proyección espinosa
Levemente festoneado y sin proceso espinoso 
TABLA 1 (continuación)

\begin{tabular}{|c|c|c|c|}
\hline Hueso & P. patagonicus & P. orbignyanus & P. isosceles \\
\hline $\begin{array}{l}\text { Metapterigoi- } \\
\text { des }\end{array}$ & $\begin{array}{l}\text { Borde anterior con concavi- } \\
\text { dad bien marcada. Margen } \\
\text { posterior con escotadura en } \\
\text { parte inferior }\end{array}$ & $\begin{array}{l}\text { Borde anterior recto. Margen } \\
\text { posterior cóncavo }\end{array}$ & $\begin{array}{l}\text { Borde anterior sinuoso. Margen } \\
\text { posterior recto }\end{array}$ \\
\hline $\begin{array}{l}\text { Hiomandibu- } \\
\text { lar (ángulo } \\
\text { posterior) }\end{array}$ & Marcadamente cóncavo & Levemente cóncavo & Recto \\
\hline $\begin{array}{l}\text { Simpléctico } \\
\text { (borde ante- } \\
\text { rior) }\end{array}$ & $\begin{array}{l}\text { Cóncavo con una proyección } \\
\text { espinosa anterior }\end{array}$ & Irregular & $\begin{array}{l}\text { Recto con una expansión lami- } \\
\text { nar con márgenes aserrados }\end{array}$ \\
\hline $\begin{array}{l}\text { Epihial (bor- } \\
\text { de dorsal) }\end{array}$ & Levemente convexo & Recto & Convexo \\
\hline $\begin{array}{l}\text { Hipohial su- } \\
\text { perior (ancho } \\
\text { en relación al } \\
\text { largo) }\end{array}$ & $66 \%$ & $80 \%$ & $53 \%$ \\
\hline $\begin{array}{l}\text { Urohial (rama } \\
\text { inferior) }\end{array}$ & $\begin{array}{l}\text { Canal longitudinal poco } \\
\text { marcado }\end{array}$ & Sin canal longitudinal & Canal longitudinal bien notorio \\
\hline $\begin{array}{l}\text { O p é r c u } 1 \text { o } \\
\text { (borde supe- } \\
\text { rior) }\end{array}$ & Con profunda escotadura. & Levemente cóncavo & Liso y recto \\
\hline $\begin{array}{l}\text { Interopérculo } \\
\text { (ancho en el } \\
\text { largo) }\end{array}$ & Cabe 1,7 veces & Cabe 1,4 veces & Cabe 2 veces \\
\hline $\begin{array}{l}\text { Tercer basi- } \\
\text { branquial }\end{array}$ & $\begin{array}{l}\text { Extremo anterior angosto, } \\
\text { con ensanchamiento en parte } \\
\text { media y adelgazamiento en } \\
\text { extremo posterior }\end{array}$ & $\begin{array}{l}\text { Extremo anterior con dos conca- } \\
\text { vidades anteriores }\end{array}$ & $\begin{array}{l}\text { Extremo anterior bien ensancha- } \\
\text { do y largo estrechamiento en re- } \\
\text { gión posterior }\end{array}$ \\
\hline $\begin{array}{l}\text { Tercer epi- } \\
\text { branquial }\end{array}$ & $\begin{array}{l}\text { Con proceso posterior espi- } \\
\text { noso y dientes pequeños }\end{array}$ & $\begin{array}{l}\text { Con proceso posterior espinoso } \\
\text { unido al hueso por una expan- } \\
\text { sión laminar }\end{array}$ & $\begin{array}{l}\text { Con proceso posterior redondea- } \\
\text { do y dientes cónicos y puntiagu- } \\
\text { dos }\end{array}$ \\
\hline
\end{tabular}

\section{CONCLUSIONES}

El estudio morfológico de los elementos óseos ha sido una herramienta indispensable para la identificación de los ítemes presa en los contenidos estomacales de animales ictiófagos (Favero et al. 2001). Por ello, las diferencias morfológicas encontradas en los componentes óseos del cráneo de tres especies de lenguados, son importantes como criterios de valor diagnóstico para la identificación de las especies de Paralichthys en el Atlántico suroccidental. Estas diferencias, conjuntamente con características morfométricas y merísticas, previamente utilizadas para la distinción de las especies de Paralichthys que ocurren en aguas argentinas y uruguayas (Díaz de Astarloa 1994), constituyen caracteres diagnósticos para distinguir a las tres especies. En porcentaje de la longitud estándar, los valores medios del diámetro orbitario $(7,4 ; 3,2 ; 5,6)$, la anchura interorbitaria $(1,4 ; 3,3 ; 1,7)$ y la longitud de la aleta pectoral $(16 ; 11,1 ; 12,8)$, entre los caracteres morfométricos, y el número de radios de las aletas dorsal $(84,2 ; 74,7 ; 81)$ y anal $(67 ; 56,1 ; 63)$ como el número de hileras de escamas de la línea lateral $(75,3 ; 98,3 ; 104)$ y el número de branquispinas $(10 ; 19,4 ; 13,1)$ entre los merísticos, han mostrado evidencias de constituir importantes caracteres para separar a Paralichthys isosceles, $P$. orbignyanus y $P$. patagonicus, respectivamente (Díaz de Astarloa 1994, Díaz de Astarloa \& Munroe datos no publicados). Los caracteres osteológicos que más distinguen a las especies de Paralichthys son los huesos paretmoides 
derecho, lacrimal izquierdo, otolitos, anguloarticular, mesopterigoides y número de dientes del dentario. En $P$. isosceles el paretmoides derecho presenta tres procesos posteriores espinosos, el brazo inferior del lacrimal izquierdo es curvo con una profunda concavidad en su cara externa, el otolito derecho es de forma cuadrangular con el borde ventral liso, la longitud del canal óseo del anguloarticular es un $21 \%$ del largo total del hueso, el borde posterior del mesopterigoides es levemente festoneado y el número de dientes en el dentario es el más alto, con un rango de 16 a 21 y un valor medio de 18,5. En $P$. orbignyanus, el paretmoides derecho presenta dos procesos posteriores espinosos, el brazo inferior del lacrimal izquierdo es recto y sin concavidad alguna en su cara externa, el otolito derecho es oblongo y el borde ventral es festoneado, la longitud del canal óseo del anguloarticular es un $38 \%$ del largo total del hueso, el borde posterior del mesopterigoides es liso con un proceso espinoso y el número de dientes en el dentario con rango de 9 a 13 y una media de 11 . En $P$. patagonicus, el paretmoides derecho presenta dos procesos posteriores espinosos, el brazo inferior del lacrimal izquierdo es recto y sin concavidad alguna en su cara externa, el otolito derecho es subtriangular y el borde ventral es liso, la longitud del canal óseo del anguloarticular es un $30 \%$ del largo total del hueso, el borde posterior del mesopterigoides es aserrado con proceso espinoso y el número de dientes en el dentario es el menor, con un rango de seis a nueve y una media de 7,4 .

Estas características conjuntamente con los caracteres morfométricos y merísticos mencionados más arriba, constituirían potenciales caracteres diagnósticos para la diferenciación de las especies de Paralichthys.

\section{AGRADECIMIENTOS}

El autor agradece sinceramente a los Dres. Fernando Cervigón, María Berta Cousseau, Daniel Figueroa, Thomas A. Munroe, Atila Gosztonyi, Amalia Miquelarena y Ricardo Perrotta por los valiosos aportes vertidos en las primeras versiones del manuscrito, a la Lic. Marta Herrera y la Cartógrafa Carmen Milloc por la confección de varias Figs., a la Sra. Marcela
Tobío por las fotografías, a los Dres. Thomas A. Munroe, Bruce B. Collette, Lisa Palmer, Debie Lofler, Gabriela Piacentino, Gustavo Chiaramonte, Izumi Nakamura, Martine Desoutter, Patrice Pruvost, José L. de Figueiredo y Gustavo Nunan por el envío de ejemplares o la ayuda prestada para examinar ejemplares durante diversas visitas a colecciones ictiológicas. Mi más sincero agradecimiento a los colegas y tripulantes de los BIP "Dr. Eduardo L. Holmberg" y "Cap. Oca Balda" del Instituto Nacional de Investigación y Desarrollo Pesquero (INIDEP) por la desinteresada colaboración en la colecta de los peces. Este trabajo fue finalizado gracias al apoyo de la Japan Society for the Promotion of Science (JSPS) y en especial al Prof. Masaru Tanaka, mediante una beca de larga duración para investigadores visitantes en la Universidad de Kyoto, Japón. Finalmente deseo expresar mi gratitud al Dr. Brian Dyer cuyos comentarios y sugerencias contribuyeron a mejorar sustancialmente el manuscrito.

\section{LITERATURA CITADA}

ALOISI PN (1990) Características morfológicas y morfométricas de los otolitos de tres especies de pejerrey de la costa marplatense. Tesis de grado, Facultad de Ciencias Exactas y Naturales, Universidad Nacional de Mar del Plata, Argentina. 21 pp.

AMAOKA K (1969) Studies on the Sinistral Flounders Found in the Waters around Japan. Taxonomy, Anatomy and Phylogeny. Journal of the Shimonoseki University of Fisheries 18: 65-340.

BALART EF (1985) Development of Median and Paired Fin Skeleton of Paralichthys olivaceus (Pleuronectiformes: Paralichthyidae). Japanese Journal of Ichthyology 31: 398-410.

BAUZÁ RULLÁN J (1957) Nueva contribución al estudio de los otolitos de peces actuales y fósiles de España. Memorias y Comunicaciones del Instituto Geológico de la Diputación Provincial de Barcelona (España) 16: 33-44

CASSELMAN JM (1983) Age and growth assessment of fish from their calcified structures: Techniques and tools. In: Proceedings of the international workshop on age determination of oceanic pelagic fishes: Tunas, billfishes and sharks. Prince and Pulos (eds). NOAA Technical Report, National Marine Fisheries Service (USA) 8: 157-165

CERVIGÓN F (1980) Ictiología Marina. Vol. 1. Editorial Arte, Caracas (Venezuela) $358 \mathrm{pp}$.

CERVIGÓN F (1985) Las especies de los géneros Achirus y Trinectes (Pisces: Soleidae) de las costas de Venezuela (Osteología, Musculatura y ligamentos fasciales, y Sistemática). Fundación Científica Los Roques. Monografía № 2. Caracas (Venezuela) 83 pp.

COLLETTE BB \& LN CHAO (1975) Systematics and Morphology of the bonitos (Sarda) and their relatives (Scombridae, Sardini). Fishery Bulletin (USA) 73: 516-625. 
COLLETTE BB \& GB GILLIS (1992) Morphology, systematics and biology of the double-lined mackerels (Grammatorcynus, Scombridae). Fishery Bulletin (USA) 90: 13-53.

DÍAZ DE ASTARLOA JM (1991) Estudios osteológicos del sincráneo y complejo caudal en dos formas nominales de Paralichthys: Paralichthys patagonicus y Paralichthys bicyclophorus. Frente Marítimo (Uruguay) 9A: 15-27.

DÍAZ DE ASTARLOA JM (1994) Las especies del género Paralichthys del Mar Argentino (Pisces, Paralichthyidae). Morfología y sistemática. Tesis doctoral. Universidad Nacional de Mar del Plata, Argentina. 194 pp.

DÍAZ DE ASTARLOA JM (1995) Variación intraespecífica del patrón de pigmentación en Paralichthys isosceles (Pleuronectiformes: Paralichthyidae) Neotrópica 41: 55-62.

DÍAZ DE ASTARLOA JM (1996) Paralichthys patagonicus Jordan, in Jordan \& Goss, 1889, a senior synonym of $P$. bicyclophorus Miranda Ribeiro, 1915 (Paralichthyidae: Pleuronectiformes) Copeia 1996: 1035-1037.

DINGERKUS G \& LH UHLER (1977) Enzime clearing of alcian blue stained whole smlall vertebrates for demostration of cartilage. Stain Technolgy (USA) 52: 229-232.

FABRÉ NN (1988) Estudio morfológico y morfométrico de los otolitos de dos especies de lenguados, Xystreuris rasile y Paralichthys isosceles (Pisces, Bothidae). Physis, Sección A (Argentina) 46: 7-14.

FABRÉ NN \& MB COUSSEAU (1990) Sobre la determinación de la edad y el crecimiento del lenguado Paralichthys isosceles aplicando retrocálculo. Revista Brasileira de Biologia 50: 345-354.

FAVERO M, S BACHMANN, S COPELLO, R MARIANO-JELICICH, MP SILVA, M GHYS, C KHATCHIKIAN \& L MAUCO (2001) Aves marinas del sudeste bonaerense, En: Iribarne O (ed) Reserva Mar Chiquita: características físicas, biológicas y ecológicas: 251-267. Editorial Martin, Mar del Plata, Argentina.

FUTCH CR, RW TOPP \& ED HOUDE (1972) Developmental osteology of the lined sole, Achirus lineatus (Pisces, Soleidae). Contributions in Marine Science (USA) 16: 33-58.

GINSBURG I (1952) Flounders of the genus Paralichthys and related genera in American waters. Fishery Bulletin (USA) 52: 267-351.

HENSLEY DA \& EH AHLSTROM (1984) Pleuronectiformes: Relationships. En: Moser HG et al (eds) Ontogeny and Systematics of Fishes: 670-687. American Society of Ichthyologists and Herpetologists. Special Publication number 1. Allen Press, Kansas, USA.

HOSHINO K \& K AMAOKA (1998) Osteology of the flounder, Tephrinectes sinensis (Lacèpede) (Teleostei: Pleuronectiformes), with comments on its relationships. Ichthyological Research 45: 69-77.

JORDAN DS \& BW EVERMANN (1898) The fishes of North and Middle America. Flatfishes. Bulletin of the United States National Museum 47: 2602-2712.

JORDAN DS \& DK GOSS (1889) A review of the flounders and soles (Pleuronectidae) of America and Europe. Annual Report of the United States Commission of Fisheries and Fish: 225-342.

KAWAMURA K \& K HOSOYA (1991) A modified double staining technique for making a transparent fishskeletal specimen. Bulletin of the National Research Institute of Aquaculture (Japan) 20: 11-18

KYLE HM (1921) The assymmetry, metamorphosis and origin of flatfishes. Philosophical Transactions of the Royal Society of London, Series B 211: 75 -129.

LEMA T DE, MFT DE OLIVEIRA \& CAS DE LUCENA (1980) Levantamento preliminar dos Pleuronectiformes do estremo sul do Brasil do Río de la Plata (Actinopterygii: Teleostei). Iheringia, Serie Zoológica (Brasil) 56: 25-52.

LEVITON AE, RH GIBBS Jr, E HEAL \& CE DAWSON (1985) Standards in herpetology and ichthyology: Part 1. Standard symbolic codes for institutional resource collections in herpetology and ichthyology. Copeia 1985: 802-832.

MAYDEN RL, \& EO WILEY (1984) A method of preparing disarticulated skeletons of small fishes. Copeia 1984: 230-232.

MENNI RC, RA RINGUELET \& RH ARÁMBURU (1984) Peces marinos de la Argentina y Uruguay. Catálogo crítico ilustrado. Claves para la determinación de familias, géneros y especies. Nombres vulgares. Glosario. Ediciones Hemisferio Sur S.A., Buenos Aires, Argentina. 360 pp.

MIRANDA RIBEIRO A (1915) Fauna brasiliense, Peixes. Tomo V (Eleutherobranchios). Arquivos do Museo Nacional Río de Janeiro 17: 19-22.

NORMAN JR (1934) A systematic monograph of the flatfishes (Heterosomata) Vol. I. Psettodidae. Bothidae. Pleuronectidae. British Museum of Natural History, London, United Kingdom. 459 pp.

OSSIAN CR (1970) Preparation of disarticulated skeletons using enzime-based laundry "pre-soakers". Copeia 1970: 190-200

PATTERSON C (1975) The braincase of Pholidophorid and Leptolepid fishes, with a review of the Actinopterygian braincase. Philosophical Transactions of the Royal Society of London, Series B 269: 275-579.

PATTERSON C (1976) Cartilage bones, dermal bones and membrane bones, or the exoskeleton versus the endoskeleton. En: Mahala A et al. (eds) Problems in vertebrate evolution. Linnean Society Symposium 4: $411 \mathrm{pp}$.

PEQUEÑO G \& E D'OTTONE (1987) Diferenciación taxonómica de los lenguados comunes de Valdivia, Chile (Osteichthyes, Bothidae). Revista de Biología Marina (Chile) 23: 107-137.

PEQUEÑO G \& R PLAZA (1987) Descripción de Paralichthys delfini n. sp., con notas sobre otros lenguados congenéricos de Chile (Pleuronectiformes, Bothidae). Revista de Biología Marina (Chile) 23: 159-172.

PÉREZ MA \& R WERNER (1986) Descripción del neurocráneo de dos especies de lenguados, Xystreuris rasile y Paralichthys isosceles. Tesis de Licenciatura, Facultad de Ciencias Exactas y Naturales, Universidad de Buenos Aires, Buenos Aires, Argentina. 35 pp.

POTTHOFF $\mathrm{T}$ (1984) Clearing and staining techniques. En: Moser HG et al. (eds) Ontogeny and systematics of fishes: 35-37. American Society of Ichthyologists and Herpetologists, Special Publication 1. Allen Press, Kansas, USA.

RINGUELET RA \& RH ARÁMBURU (1960) Peces marinos de la República Argentina. Agro (Agentina), año II, 5. $141 \mathrm{pp}$.

ROJO AL (1988) Diccionario enciclopédico de anatomía de peces. Monografías del Instituto Español de Oceanografía № 3.566 pp.

ROUX C (1973) Poissons Téléostéens du plateaux continental brésilien. Résultats scientifiques des campagnes de la "Calypso", X. Annales de 1'Institut 
Océanographique de Monaco. Fascicule Supplémentaire 49: 23-207.

TOPP RW \& CF COLE (1968) An osteological study of the sciaenid genus Sciaenops Gill (Teleostei, Sciaenidae). Bulletin of Marine Science (USA) 18: 902-945.

VILELA NN (1988) Morfología y morfometría de los otolitos sagita de peces del mar argentino. Tesis de Licenciatura, Facultad de Ciencias Exactas y Naturales, Universidad Nacional de Mar del Plata, Mar del Plata, Argentina. $31 \mathrm{pp}$.

VOLPEDO AV \& DD ECHEVERRÍA (1997) Morfología de las sagittae de lenguados del mar argentino (Bothidae, Paralichthyidae y Achiropsettidae). Thalassas (España)13: 113-126.

VOLPEDO AV \& DD ECHEVERRÍA (2000) Catálogo y claves de otolitos para la identificación de peces del Mar Argentino. I. Peces de importancia comercial. Editorial Dunken, Buenos Aires, Argentina, 90 pp.
VOLPEDO AV \& DD ECHEVERRÍA (2001) Morfología y morfometría de las sagittae de sciaenidos marinos del norte de Perú. Boletín de la Sociedad Biológica de Concepción (Chile) 72: 147-154.

VOLPEDO AV \& DD ECHEVERRÍA (2003) Ecomorphological patterns of the sagitta in fish on the continental shelf off Argentine. Fisheries Research 60: 551-560.

WATT J, GJ PIERCE \& PR BOYLE (1997) Guide to the identification of North Sea fish using premaxillae and vertebrae. ICES Co-op Research Report 220: 1-231.

WOOLCOTT WS, C BEIRNE \& WM HALL (1968) Descriptive and comparative osteology of the young of three species of flounders, genus Paralichthys. Chesapeake Science 9: 109-120.

YAZDANI GM (1969) Adaptation in the jaws of flatfish (Pleuronectiformes). Journal of Zoology 159: 181222.

Editor Asociado: Brian Dyer

Recibido el 16 de julio de 2003; aceptado el 3 de enero de 2005

ANEXO 1

Abreviaturas usadas para los huesos

Abbreviations used for bone names

\begin{tabular}{llll}
\hline A. ar. & Anguloarticular & La. & Lacrimal \\
Bb. 1-3 & Basibranquiales & Li. & Ligamentum \\
Bs. & Basioccipital & Me. & Mesetmoides \\
C.Mck. & Cartílago de Meckel & Msp. & Mesopterigoides \\
Cb. 1-5 & Ceratobranquiales & Mtp. & Metapterigoides \\
Cd. & Coracoides & Mx. & Maxilar \\
Cl. & Cleitrum & Na. d. & Nasal derecho \\
Cor. & Coronomeckeliano & Na. i. & Nasal izquierdo \\
Cu. & Cuadrado & Op. & Opérculo \\
Ch. & Ceratohial & Pa. & Paretmoides \\
De. & Dentario & Pc. & Proótico \\
Eb. 1-4 & Epibranquiales & Pcl. i. & Postcleitrum inferior \\
Ect. & Ectopterigoides & Pcl. s. & Postcleitrum superior \\
Eh. & Epihial & Pe. & Postemporal \\
Ep. & Epioccipital & Pf. & Pterosfenoides \\
Es. & Esfenótico & Pi. & Parietal \\
Esc. & Escápula & Pl. & Palatino \\
Et. & Extraescapular & Po. & Preopérculo \\
Ex. & Exoccipital & Pr. & Premaxilar \\
Fb. 1-4 & Faringobranquiales & Ps. & Parasfenoides \\
Fm. & Foramen magnum & Pt. & Pterótico \\
Fr. & Frontal & R. Pec. & Radiales pectorales \\
H. 1-5 & Hipurales & Ra. br. & Radios branquiostegos \\
Hb. 1-3 & Hipobranquiales & Rad. P. & Radios pectorales \\
Hh. i. & Hipohial inferior & Ret. & Retroarticular \\
Hh. s. & Hipohial superior & Scl. & Supracleitrum \\
Hio. & Hiomandibular & Si. & Simpléctico \\
I. or. & Infraorbitarios & Sop. & Subopérculo \\
Ih. & Interhial & Su. & Supraoccipital \\
In. & Intercalar & Uh. & Urohial \\
Iop. & Interopérculo & Vo. & Prevómer \\
\hline
\end{tabular}

